\title{
Decoding motor neuron behavior for advanced control of upper limb prostheses
}

\author{
Dissertation \\ zur Erlangung des humanwissenschaftlichen Doktorgrades \\ in der Medizin \\ der Georg-August-Universität Göttingen
}

vorgelegt von

Tamás Kapelner

aus Budapest

Göttingen, 2016 


\section{Betreuungsausschuss}

$\begin{array}{ll}\text { Erstbetreuer } & \text { Prof. Dario Farina* } \\ \text { Weitere Betreuer/innen } & \text { Prof. Gerlind Plonka-Hoch } \\ & \text { Prof. David Liebetanz* }\end{array}$

*Gutachter

Weitere Mitglieder der Prüfungskommission

Prof. Hansjörg Scherberger

Prof. Alexander Gail

Prof. Florentin Wörgötter

Tag der mündlichen Prüfung: 25.10.2016 
I hereby declare that this thesis, including all tables and graphics, is original and written by me independently, with no other sources than quoted. Nor the whole thesis neither parts of it were used in any other submitted doctoral thesis.

The research presented in this thesis was financed by the German Academic Exchange Service Research Grant for Doctoral Candidates and Young Academics and Scientists (Grant \#57076385).

Göttingen, August 29, 2016

Tamás Kapelner 


\section{ACKNOWLEDGEMENTS}

First and foremost I would like to thank Dario Farina for accepting me as a $\mathrm{PhD}$ candidate out of the blue, and granting me the opportunity to take part in outstanding research. He made it possible for me to participate in the scientific discussion on conferences and symposia, and supported my work with his invaluable advice and expertise over the course of these three years.

I am also thankful for the mentorship of Ning Jiang in the first half of my project, especially because I suspect we would not know if the results of this thesis were significant without his statistical coaching.

I would also like to thank the DAAD for providing funding for my research, and the members of my Thesis Committee, Prof. Gerlind Plonka-Hoch and Prof. David Liebetanz, for co-supervising the $\mathrm{PhD}$ project.

I owe many thanks to all my colleagues at the Institute of Neurorehabilitation Systems for their valuable input on several matters during department meetings, symposia and discussions in the kitchens (of both buildings). They have sacrificed their time to take part in my experiments, and also to move furniture with me in all shapes and sizes. I had the privilege to enjoy movies, football games, karaoke nights and barbecues with this extraordinary group of people.

My special thanks go to people who contributed to this thesis by performing experiments, assisting me in data analysis, or letting me sleep on the floor during trips: Ivan Vujaklija, Francesco Negro, Massimo Sartori and Utku Yavuz. I am also grateful for the daily lessons in Italian from Silvia Muceli and Antonietta Stango.

The person who has contributed the most to this thesis was undoubtedly Nikoletta Vörös, supporting me with her love and encouragement from the scholarship application to this moment, for which I am deeply thankful. This work is dedicated to her and my family, who supported me relentlessly as well during these years away from home.

Thank you all! 

One of the main challenges in upper limb prosthesis control to date is to provide devices intuitive to use and capable to reproduce the natural movements of the arm and hand. One approach to solve this challenge is to use the same control signals for prosthesis control that our nervous system uses to control its muscles. This thesis aims to investigate the possibility of natural, intuitive prosthesis control using neural information obtained with available surface EMG decomposition methods. In order to explore all aspects of such a novel approach, a series of five studies were performed with the final goal of implementing a proof of concept and comparing its performance with state of the art myoelectric control. The performed investigations revealed important insights in motor unit physiology after targeted muscle reinnervation, EMG decomposition in dynamic voluntary contractions of the forearm, and the properties and challenges of neural information based prosthesis control. The main outcome of the thesis is that neural information based prosthesis control is capable to outperform myoelectric approaches in pattern recognition, linear regression and nonlinear regression, as determined by offline performance comparisons. The final proof of concept for this novel approach was a robust regression method based on neuromusculoskeletal modeling. The kinematics estimation of the proposed approach outperformed EMG-based nonlinear regression in both able-bodied subjects and patients with limb deficiency, indicating that using neural information is a promising avenue for advanced myoelectric control. 
1 Introduction $\quad 1$

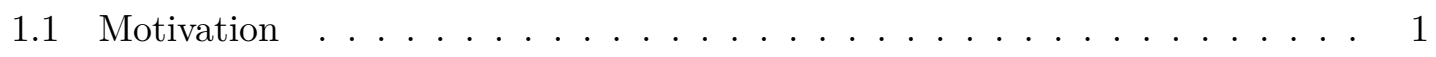

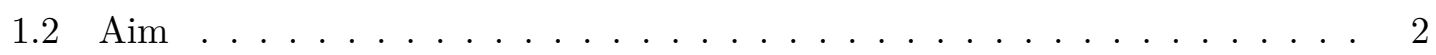

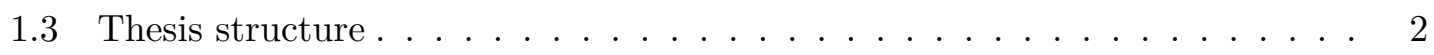

2 Physiological background 5

2.1 The neural drive to muscle $\ldots \ldots \ldots \ldots \ldots$

2.2 The surface electromyogram . . . . . . . . . . . . . . 7

2.3 EMG decomposition . . . . . . . . . . . . . . . . 8

2.4 Myoelectric control methods . . . . . . . . . . . . . . . . . . . 9

2.4.1 Conventional control . . . . . . . . . . . . . . . . 10

2.4.2 Pattern recognition . . . . . . . . . . . . . . . . 11

2.4 .3 Regression . . . . . . . . . . . . . . . . . 12

2.5 Neural information in myoelectric control . . . . . . . . . . . . . . . 13

2.6 Targeted muscle reinnervation . . . . . . . . . . . . . . . . . . 14

$\begin{array}{lll}3 & \text { Thesis contribution } & \mathbf{1 7}\end{array}$

3.1 Study 1: Electrophysiological changes in motor units after TMR . . . . . . 19

3.1 .1 Introduction . . . . . . . . . . . . . . . . . . . 19

3.1 .2 Methods . . . . . . . . . . . . . . . . . . . . . . . . . . 19

3.1 .3 Results . . . . . . . . . . . . . . . . . 23

3.1.4 Discussion . . . . . . . . . . . . . . . . . . . . . . . 24

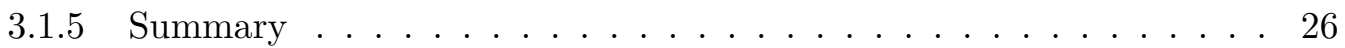

3.2 Study 2: Pattern recognition based on neural information . . . . . . . . 28

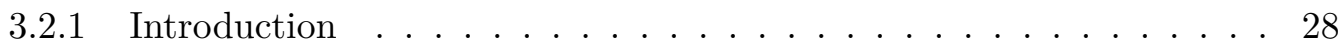

3.2 .2 Methods . . . . . . . . . . . . . . . . . . . . . 28

3.2 .3 Results . . . . . . . . . . . . . . . . . . 31

3.2 .4 Discussion . . . . . . . . . . . . . . . . . . . . 31 
3.2 .5 Summary . . . . . . . . . . . . . . . . . 33

3.3 Study 3: The feasibility of EMG decomposition during voluntary contractions 34

3.3 .1 Introduction . . . . . . . . . . . . . . . . . . . 34

3.3 .2 Methods . . . . . . . . . . . . . . . . . . . 34

3.3 .3 Results . . . . . . . . . . . . . . . . . . . 37

3.3 .4 Discussion . . . . . . . . . . . . . . . . . . . . . 39

3.3 .5 Summary . . . . . . . . . . . . . . . . . 41

3.4 Study 4: Linear regression based on neural information . . . . . . . . . . . 42

3.4 .1 Introduction . . . . . . . . . . . . . . . . . . . . . . 42

3.4 .2 Methods . . . . . . . . . . . . . . . . . . . 43

3.4 .3 Results . . . . . . . . . . . . . . . . . . . . 47

3.4 .4 Discussion . . . . . . . . . . . . . . . . . 48

3.4 .5 Summary . . . . . . . . . . . . . . . . . 50

3.5 Study 5: Neuromusculoskeletal modeling for prosthesis control . . . . . . . . 52

3.5.1 Introduction . . . . . . . . . . . . . . . . . . . . 52

3.5 .2 Methods . . . . . . . . . . . . . . . . . . 52

3.5.3 Results . . . . . . . . . . . . . . . . 56

3.5.4 Discussion . . . . . . . . . . . . . . . . 57

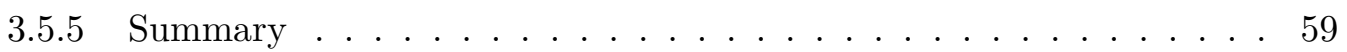

4 General discussion $\quad 61$

$\begin{array}{ll}\text { Bibliography } & 65\end{array}$

$\begin{array}{ll}\text { Appendix } & \mathbf{7 7}\end{array}$

A EMG decomposition using the CKC method . . . . . . . . . . . . . . 77

B Detailed results of Section $3.4 \ldots \ldots \ldots \ldots$. . . . . . . . . . 79

B.1 Feature . . . . . . . . . . . . . . . . 79

B.2 Ramp duration . . . . . . . . . . . . . . . . 80

B.3 Movement range . . . . . . . . . . . . . . . . . . . 82 
2.1.1 An illustration of the motor unit structure . . . . . . . . . . . . 6

2.4.1 Schematic representation of myoelectric control . . . . . . . . . . . 10

2.4.2 Conceptual depiction of conventional myoelectric control methods . . . . . 10

2.4.3 Conceptual depiction of pattern recognition methods . . . . . . . . . . . . . 11

2.4.4 Conceptual depiction of regression methods . . . . . . . . . . . . . . 12

2.6.1 Illustration of the TMR procedure . . . . . . . . . . . . . . . . . . 14

3.1.1 Electrode locations for the TMR patients . . . . . . . . . . . . . . . 21

3.1.2 Multichannel MUAP shape and the corresponding interpolated RMS map . 22

3.1.3 Representation of EMG decomposition . . . . . . . . . . . . . . . . . . . . 24

3.1.4 Mean and standard deviation of the investigated motor unit properties . . . 25

3.1.5 Spatial positions of the motor unit surface areas . . . . . . . . . . 25

3.2.1 An illustration of region pooling . . . . . . . . . . . . . . . 30

3.2.2 Block diagrams of the two compared pattern recognition approaches (@2015

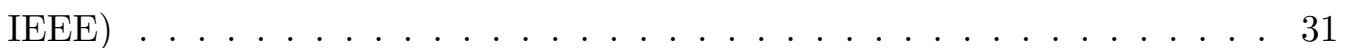

3.2.3 Confusion matrices for the classification of motor tasks . . . . . . . . . . 32

3.3.1 An illustration of the EMG recording and motion capture system, the signal processing steps and the visual feedback . . . . . . . . . . . . . 35

3.3.2 A representative example of the results of EMG decomposition (@2016 IEEE) 37

3.3.3 Surface representation of the motor tasks (@2016 IEEE) . . . . . . . . . . . 39

3.3.4 Histograms of the normalized angles at recruitment (@2016 IEEE) . . . . . 40

3.4.1 A representative example of the comparison method based on MUAP shapes 44

3.4.2 The proposed dimensionality reduction model and the physiological model that it is inspired by . . . . . . . . . . . . . . . . . . . 44 45

3.4.3 Representative examples of the training of the activations estimated by groups of motor units . . . . . . . . . . . . . . . 46

3.4.4 An overview of the results combining the data of all subjects . . . . . . . 48

3.5.1 An illustration of the proposed NMS modeling framework . . . . . . . . . 53 
3.5.2 Illustration of the experiment protocol and data acquisition (@2016 IEEE) . 54

3.5.3 Block diagram of the proposed NMS model (@2016 IEEE) . . . . . . . . . . 54

3.5.4 The performance of the investigated methods across subjects (@2016 IEEE) 57

3.5.5 An example of estimation results (O2016 IEEE) . . . . . . . . . . . . 58

B.1 Performance comparison between features at the full range of movement . . 79

B.2 Performance comparison between features at the $20 \%$ movement range . . . 80

B.3 Performance comparison between ramp durations at the full movement range 81

B.4 Performance comparison between ramp durations at the $20 \%$ movement range 81

B.5 Performance comparisons between the full and the reduced movement range 82 
3.1 Age, gender and amputation details of TMR patients . . . . . . . . . . . . 20

3.2 Number of decomposed spike trains per task for each subject . . . . . . . . 23

3.3 Number of decomposed spike trains and discharge statistics for the ablebodied subjects (@2016 IEEE) _ . . . . . . . . . . . . . . . . 38

3.4 Number of decomposed spike trains and discharge statistics for the subjects with limb-deficiency $(\bigcirc 2016$ IEEE) . . . . . . . . . . . . . . . . . . . 38

3.5 The mean and standard deviation of decomposed spike trains . . . . . . . . 47

3.6 The number of decomposed spike trains per DoF for each subject (@2016

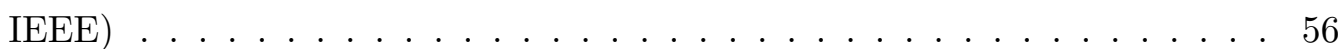

3.7 The average number of spike trains assigned to each muscle (@2016 IEEE) . 56 
ANN

ANOVA

CMC

DoF

DSC

ECU

ECRL

EMG

FCR

FCU

MUAP

NMS

pps

PT

RMS

SIL

SIR

SUP

TD

TMR
Artificial Neural Network

Analysis of Variance

Computed Muscle Control

Degree of Freedom

Decomposed Spike Counts

Extensor Carpi Ulinaris

Extensor Carpi Radialis Longus

Electromyogram

Flexor Carpi Radialis

Flexor Carpi Ulinaris

Motor Unit Action Potential

Neuromusculoskeletal

pulses per second

Pronator Teres

Root Mean Square

Silhouette

Signal to Interference Ratio

Supinator

Time Domain

Targeted Muscle Reinnervation 


\subsection{Motivation}

Our hand is our most powerful instrument of interaction with the world. It is capable of excelling at a wide range of dynamic and precise tasks, such as playing the piano, performing surgery or manipulating small objects. This functionality is made possible by a sophisticated biomechanical system that has 27 degrees of freedom (DoF) actuated by more than 30 muscles, which are controlled by the largest of brain areas devoted to a specific motor function (ElKoura and Singh 2003; Penfield and Boldrey 1937).

Losing this essential instrument has a profound effect on the quality of life. In addition to having to cope with physical disability, patients with limb loss experience pain, sleeping, and emotional problems, decreased energy levels, and social isolation (Atroshi and Rosberg 2001). This is especially concerning since most upper limp amputations occure at a young age. Depending on the country, there are an estimated 7-12 people out of 100000 suffering upper limb loss each year, two thirds of the time under the age of 40 , and $90 \%$ of these cases are caused by trauma or congenital deficiencies (Demet et al. 2003; Durance and O'shea 1988; Østlie et al. 2011; Ziegler-Graham et al. 2008).

An essential goal in improving the quality of life of these patients is the replacement of the lost function, which has also been linked with the reduction of phantom limb pain through preventing cortical reorganization (Atzori and Müller 2015; Hargrove, Lock, and Simon 2013; Kuiken et al. 2004). The importance of replacement was recognized as early as in 1000 B.C. in Egypt, as demonstrated by the introduction of the first cosmetic prosthesis (Nerlich et al. 2000). Throughout history, there are more and more examples of engineering solutions with increasing complexity and functionality, including the first body-powered prosthesis from 1812 (Gaine, Smart, and Bransby-Zachary 1997).

Today state of the art upper limb prostheses are controlled via a myoelectric interface. The muscle activity in the residual limb is detected using the surface electromyogram 
(EMG), and used for driving a prosthetic device. Since the residual muscles generate myoelectric signals in response to the drive received from the central nervous system, in this application they are used as biological amplifiers of neural signals. This concept is fully realized in a recent surgical method called targeted muscle reinnervation (TMR), whereby the control over muscles lost during the amputation is restored by connecting their nerves to accessory muscles. This approach enables patients to use complex prosthetic devices even with above-elbow (transhumeral) amputations, where the muscles used for myoelectric interfacing are typically missing (Hargrove, Lock, and Simon 2013; Kuiken et al. 2004).

There is a wide variety in commercially available prosthetic devices. Although the most advanced ones are capable of actuating more than 20 DoFs, enabling independent movements of individual fingers (Atzori and Müller 2015), in practice their function is limited to relatively few pre-defined movements. This limitation is imposed by current commercial control methods unable to extract and utilize the control information required to perform natural movements (Jiang et al. 2014).

The shortcomings of myoelectric control algorithms are best demonstrated by prosthesis abandonment. Recent studies reported that overall $20 \%$ of myoelectric prosthesis users either reject wearing a prosthesis or completely abandon their use, and another $10 \%$ do not use their prosthesis frequently (Biddiss and Chau 2007a; Østlie et al. 2012). Other reports indicated overall rejection rates of 35\% (Wright, Hagen, and Wood 1995). Besides design issues such as comfort, weight, warm-up or appearance, the most prevalent factors in rejection or abandonment were the lack of functionality and difficult control (Biddiss and Chau 2007a).

\section{$1.2 \quad \operatorname{Aim}$}

Providing fluent and natural control is one of the main challenges in prosthetic technology (Jiang et al. 2012b). Natural movements of our hand are fluent and effortless because they are controlled directly by our central nervous system. Accordingly, one way to mimic the natural behavior is to provide the same neural control signals for the prosthetic device as those that control the healthy hand. Recent advances in signal processing made it possible to extract neural information non-invasively from the surface EMG, referred to as surface EMG decomposition (Farina et al. 2014; Holobar and Zazula 2007). The aim of this PhD project is therefore the following:

To investigate whether natural, intuitive prosthesis control can be achieved using neural information obtained with available surface EMG decomposition methods, and to develop a control method as a proof of concept for this novel approach.

\subsection{Thesis structure}

To define the concepts, methods, and past research results used in the remainder of the thesis, Chapter 2 briefly describes the generation of muscle force and its modeling, the 
generation of myoelectric signals, the basics of EMG decomposition, and the characteristics of current myoelectric control methods.

Chapter 3 describes the line of inquiry I have pursued in the $\mathrm{PhD}$ project in the form of several individual studies. The objectives, methods and results of these studies will be discussed in detail. This chapter contains the main contributions of the $\mathrm{PhD}$ thesis. Chapter 4 elaborates and connects all points mentioned in the individual studies, and summarizes their contributions in the context of the proposed framework and that of the related research from Chapter 2. This constitutes the discussion of the final results of the $\mathrm{PhD}$ project. 


\section{PHYSIOLOGICAL BACKGROUND}

\subsection{The neural drive to muscle}

Human movement is realized by skeletal muscles controlled by the central nervous system. The functional unit of skeletal muscle is the motor unit, made of an alpha motor neuron in the spinal cord and the muscle fibers innervated by its axon (Figure 2.1.1). The group of muscle fibers innervated by a single neuron is called a muscle unit. Discharges of the motor neuron travel along its axon to the neuromuscular junction and cause contractions in the muscle unit, generating force. The force generated by the whole muscle is controlled by its neural drive, which comprises the discharges of all its innervating motor neurons, i.e. the motor pool or motor nucleus of the muscle (Enoka 2008).

The maximal force that a muscle unit is capable to exert depends on a number of factors (Roberto Merletti and Dario Farina 2016). The most important of these is the innervation number, that is the number of muscle fibers within the unit (De Zepetnek et al. 1992). In general, more fibers are able to produce more force, thus larger motor units are also stronger.

The distribution of motor unit innervation numbers within a muscle depends on muscle function. Innervation numbers tend to be greater in muscles used for stronger crude movements, whereas muscles having to perform fine-tuned, precise movements tend to contain motor units with smaller innervation numbers (Enoka 2008).

It is worth noting that the maximal muscle unit force also depends on the cross-sectional area of the fibers as well as their specific force (De Zepetnek et al. 1992; Kanda and Hashizume 1992).

The force exerted by a motor unit at a given time depends on the discharge rate of the motor neuron and the length and shortening velocity of the muscle fibers. Generally, the generated force increases monotonically and non-linearly with an increasing discharge rate (De Luca and Contessa 2012; De Luca and Erim 1994; Moritz et al. 2005). At a given 


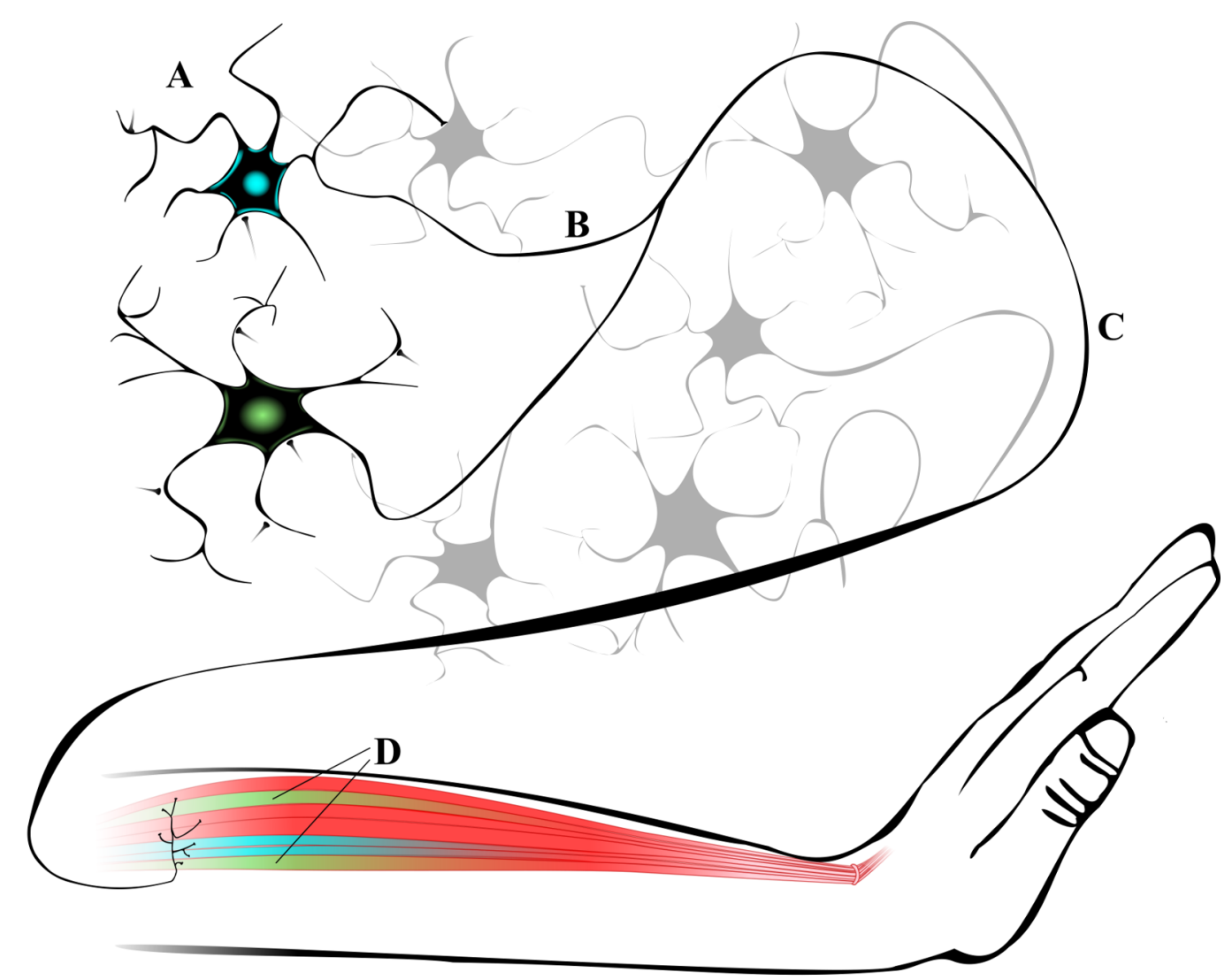

Figure 2.1.1: An illustration of the motor unit structure. The motor neuron (A) is connected to fibers in the muscle (D) through its axon (B) that is a part of a motor nerve (C) containing many axons. Neurons and fibers belonging to two different motor units are colored in this illustration (blue and green). In this notation all muscle fibers with a given color (D) comprise the muscle unit of the corresponding motor neuron. All motor neurons innervating the muscle (blue, green and gray) constitute the motor pool of the muscle. Note that the axons of motor nerves are myelinated which is not illustrated here for simplicity.

discharge rate, the generated force depends on fiber length and shortening velocity, as defined by length-force and velocity force relationships (Roberto Merletti and Dario Farina 2016). These are attributable to the cellular structure and histochemical composition of muscle fibers, as well as to biochemical changes during lengthening and shortening contractions (Colombini et al. 2007; Filippi and Troian 1994; Lieber and Ward 2011; Petit, Giroux-Metges, and Gioux 2003).

Muscle force is regulated by the central nervous system by two mechanisms: changing the number of active motor units, referred to as recruitment, and changing the rate of the action potential discharges, referred to as rate coding. These mechanisms are concurrently present, although their relative weight is different in the case of different muscles and tasks: the force of smaller muscles is mainly regulated via rate coding, since all motor units are already recruited at lower contraction forces, while for larger muscles recruitment is the dominant factor in force generation (De Luca et al. 1982; Kukulka and Clamann 1981).

The order in which motor units are recruited is relatively fixed during voluntary contractions (Henneman 1957). It occurs according to the size principle, that is small motor units are recruited first, followed by the larger ones. This happens because motor unit 
size is associated with the cellular size of the motor neuron, which defines its response to stimulation: smaller neurons have a higher excitability than larger ones, and thus will be excited by smaller stimuli at smaller exerted forces (Henneman, Somjen, and Carpenter 1965).

The force at which a motor unit is recruited is called the recruitment threshold of the unit. The distribution of recruitment thresholds in a motor pool is exponential, with most motor units exhibiting lower recruitment thresholds (Fuglevand, Winter, and Patla 1993). Changes in recruitment order can occur, however, during synergistic muscle movements (Desnedt and Gidaux 1981), during dynamic contractions (Kossev and Christova 1998), or due to changes in afferent feedback (Kanda and Desmedt 1983).

Because motor unit force is related to the discharge rate, rate coding is also used by the central nervous system to modulate muscle force. A common setting to investigate rate coding is during contractions with constant muscle length (isometric contractions) with slow changes in force. During an isometric contraction with increasing muscle force, motor units with lower recruitment thresholds exhibit larger initial discharge rates than later recruited motor units. As the force increases, so does the discharge rate of all units, whereby later recruited units arrive at larger maximal rates, typically around 30-50 pulses per second (pps) (De Luca and Contessa 2012). During fast isometric contractions maximal discharge rates can be twice as high, depending on the level of training (Cutsem, Duchateau, and Hainaut 1998). In shortening and lengthening contractions rate coding is typically different both in terms of discharge rates and discharge patterns, depending on the task requirement (Duchateau and Enoka 2008; Kossev and Christova 1998). There are also notable differences in rate coding during both ballistic and sustained contractions (Bigland-Ritchie et al. 1983; Desmedt and Godaux 1977; Riley et al. 2008).

\subsection{The surface electromyogram}

As mentioned above, each discharge of a motor unit determines an action potential that travels down the motor axon to the neuromuscular junction. Here, due to the high synaptic reliability of the neuromuscular junction, each potential evokes a muscle fiber action potential along each fiber, causing the muscle fibers to contract (Enoka 2008). These muscle fiber action potentials generate extracellular field potentials that can be measured on the surface of the skin (Farina and Merletti 2001). In this context the tissue layers between the fiber and the recording electrode are a volume conductor, through which the action potential reaches the skin surface. Since the volume conduction can be described mathematically as a filter applied to the action potential, it is commonly referred to as the tissue filter (Farina and Merletti 2001).

The signal recorded on the skin surface in response to a single discharge of a motor neuron is referred to as the motor unit action potential (MUAP). It is the global extracellular field potential from all activated muscle fibers, filtered by the volume conductor between the muscle fibers and the recording electrode. Thus, its shape is influenced by several factors, such as fibre distribution within the muscle, tissue filter characteristics, innervation 
number, contraction type, electrode characteristics etc (Fuglevand et al. 1992).

The surface EMG is a mixture of consecutive MUAPs (MUAP trains) originating from multiple motor units. Neglecting various sources of noise that can affect the signal (Day 2002), it can formally be written as:

$$
x(t)=\sum_{i=1}^{N} s_{i}(t) * h_{i}(t),
$$

where $x$ is the EMG signal, $N$ is the number of motor units observed by the electrode, $h_{i}$ is the MUAP shape as detected on the surface, and $s_{i}$ is the spike train containing consecutive discharges of the $i$-th motor unit (Holobar and Zazula 2007). Since the distance between neighboring muscles and the recording electrode is generally similar, the volume conductor effect typically allows for signals originating from multiple muscles to be present in one EMG channel, referred to as crosstalk (Solomonow et al. 1994). Indeed, in (2.1) there is no restriction to what muscles the $\mathrm{N}$ motor units have to reside in. Crosstalk is especially important for the interpretation of EMG signals during synergistic activation of multiple muscles, such as voluntary contractions of forearm muscles (De Luca and Merletti 1988).

Another characteristic of the EMG signal is its nonlinear relationship with muscle force. As indicated by (2.1), the EMG signal is an algebraic sum of MUAP trains. However, most individual $h_{i}$ MUAP shapes are bi- or triphasic, thus adding their negative phase to the sum reduces its amplitude (De Luca 1979). This is referred to as amplitude cancellation, and causes non-linearity in the EMG amplitude-force relationship (Farina, Merletti, and Enoka 2004).

Further nonlinearities are introduced by the previously mentioned force-length and force-velocity relationships, by the angle between muscle fiber and the tendon (pennation angle), and by the electromechanical delay (Buchanan et al. 2004). Nonlinearity in their relation always has to be considered when estimating muscle activity based on EMG amplitude.

\subsection{EMG decomposition}

There are several EMG decomposition methods aiming to extract discharges of motor units from EMG signals automatically (De Luca et al. 2015; Holobar and Zazula 2007; Nawab, Chang, and De Luca 2010). A majority of these employ machine learning and signal processing techniques to decompose EMG recordings in an offline (non real-time) manner.

Depending on the investigated muscle, electrode type and contraction strength, such methods are able to decompose up to 20 motor units per contraction with high accuracy, although there is no clear consensus on methods for validation of accuracy (De Luca and Nawab 2011; Farina and Enoka 2011; Farina, Merletti, and Enoka 2015; Holobar et al. 2009; Nawab, Chang, and De Luca 2010). In general, decomposition accuracy and the number of theoretically decomposable units increase with increasing number of recording 
electrodes (Farina et al. 2008).

In this thesis the Convolution Kernel Compensation (CKC) method or an extension of it was used for surface EMG decomposition (Holobar and Zazula 2007; Negro et al. 2016). This method was validated in a number of muscles and recording conditions, and used for neurophysiological studies to investigate human motor control (Hamid R. Marateb et al. 2011; Holobar, Minetto, and Farina 2014; Holobar et al. 2009; Laine, Yavuz, and Farina 2014; Negro, Holobar, and Farina 2009). Although in this original form the method is computationally expensive and therefore offline, it is possible to implement within time constraints of a real-time application (Chu, Moon, and Mun 2006). Details about the algorithm can be found in the Appendix A.

Similarly to other decomposition methods, CKC makes the assumption that MUAP shapes do not change over time, which condition is only true for isometric contractions with constant force. While investigating isometric conditions also gave us important insights on motor control (De Luca and Contessa 2012; De Luca and Erim 1994; Farina, Negro, and Dideriksen 2014; Negro, Holobar, and Farina 2009), most of our muscle contractions are dynamic.

Dynamic contractions represent a more complex decomposition problem, because of changes in the MUAP shape during the contraction (De Luca et al. 2015). These occur due to the movement of the muscle fibers relative to the electrode surface, to the shortening of the fibers, and to fatigue (Hodson-Tole and Wakeling 2009; Sandercock et al. 1985).

EMG decomposition also provides us a means to extract the MUAP shapes from experimental EMG signals, using the spike triggered averaging technique (Hu, Rymer, and Suresh 2013). It is based on the fact that according to (2.1), at each spike of the $i$-th motor unit the MUAP shape $h_{i}$ directly appears in the EMG signal, given that there are no other spikes within a duration corresponding to the length of $h_{i}$. Thus, in the noiseless case the portion of the EMG around each spiking instant contains the MUAP shape of the corresponding motor unit.

Due to noise and the superimposition of MUAPs, however, the MUAP shapes extracted in this manner contain artifacts. To reduce noise we can extract the MUAP shapes around every spiking instance (using a pre-defined observation window), and take the average of the waveforms, obtaining the spike triggered average. This can be done for each EMG channel, obtaining a multichannel representation of the MUAP. This technique will be used in this thesis to investigate MUAP properties.

\subsection{Myoelectric control methods}

Myoelectric prostheses are controlled using EMG signals recorded from residual muscles after an amputation. Although there are notable differences between control methods in several aspects, all methods rely on the following general structure to control the prosthetic device (Fougner et al. 2012) (Figure 2.4.1).

First, myoelectric signals are recorded, whereby the exact type and structure of the electrode can vary, and in general all control methods can be used with any EMG recording 


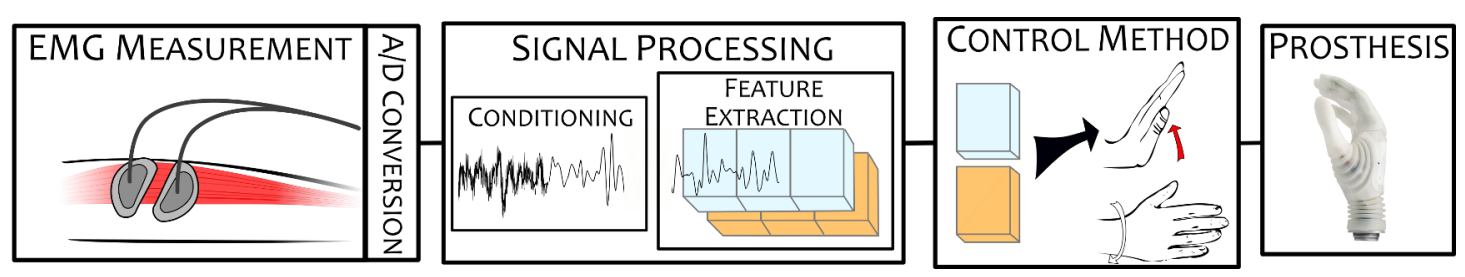

Figure 2.4.1: Schematic representation of myoelectric control. The EMG recording step is illustrated using the most commonly used surface bipolar measurement, but many other surface or intramuscular techniques can be used. The conditioning step attenuates noise and pre-processes the signal for feature extraction. Features are represented as colours, whereby each box is one numerical value of a feature calculated within an observation window. The control method chooses the action to take by the prosthesis based on the features. The image of the Michelangelo Hand prosthesis (right) is a property of and is provided by OttoBock GmbH.

system. Then, the signal is digitized and conditioned using low- or band-pass filters. The processing of the signal is carried out within observation windows, with a typical window length of 100-250 ms with some overlap between consecutive windows, to facilitate smooth changes in control (Chu, Moon, and Mun 2006; Englehart and Hudgins 2003; Li, Schultz, and Kuiken 2010; Smith et al. 2011). For each window, features are calculated to describe the characteristics of the signal and used to generate control commands for the prosthesis.

In the following the basic idea and mode of operation of different myoelectric control methods will be introduced, including their corresponding signal processing steps and their performance in replacing lost limb function.

\subsubsection{Conventional control}

In commercially available prostheses myoelectric control typically relies on two electrodes over an antagonistic muscle pair. The two EMG channels control one DoF at a time, so that one direction of the DoF is actuated proportionally to the EMG amplitude of one of the channels (Figure 2.4.2). This approach is also referred to as dual-site control, industrial state-of-the-art or amplitude control (Jiang et al. 2012b; Lake and Miguelez 2003; Young et al. 2014).

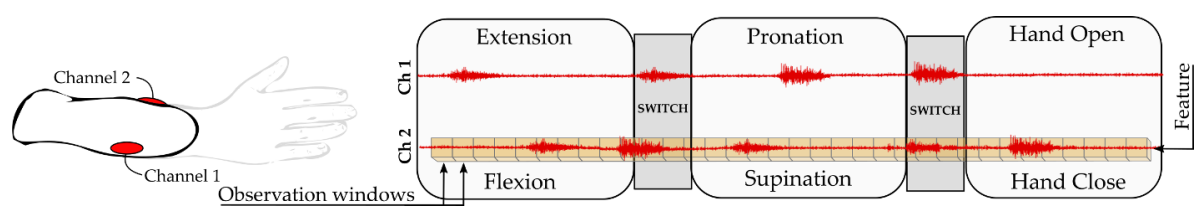

Figure 2.4.2: Conceptual depiction of conventional myoelectric control methods. The movement performed by the prosthesis (gray boxes) is regulated by the amplitude of the EMG channels (red) as a feature (orange). Channel 1 activates extension, and channel 2 flexion. For other DoFs, such as pronation/supination, a switch has to take place, which in this example consists of a simultaneous activation (co-contraction) on both channels. After the switch, channels 1 and 2 will activate pronation and supination of the prosthesis, respectively. Other DoFs can be reached by consecutive co-contractions. DoFs are typically organized in a circular manner, $i$. e. in this example performing a switch after hand open/close will activate flexion/extension again, etc. 
The method is cumbersome to adapt for multi-DoF devices, since it requires switching between actuated DoFs by performing special contractions, pressing a button etc. (Figure 2.4.2). The need for frequent switching leads to a slow and unintuitive control, which requires extensive training and imposes a considerable cognitive load during everyday tasks.

Moreover, because of the high cognitive load it is not feasible to directly actuate all DoFs of a prosthetic hand, and devices are constrained to a set of pre-defined motions. This limits the usability of some hands that in principle would be capable to provide natural control (Schulz 2011; Troccaz and Connolly 2008; Van Der Niet Otr et al. 2010). As mentioned in the introduction, the difficulty of prosthesis use and the lack of functionality are reported to be the main reasons of myoelectric prosthesis abandonment by users (Biddiss and Chau 2007a; Biddiss and Chau 2007b).

\subsubsection{Pattern recognition}

To reduce the cognitive load of prosthesis control during activities of daily living, the pattern recognition approach was proposed (Hudgins, Parker, and Scott 1993). Pattern recognition uses machine learning methods to predict the intended movement from a global multichannel EMG signal.

Formally this corresponds to a classification problem, i. e. to assign an observation to one of several classes on the basis of previous observations. Thus, this machine learning method is supervised, i. e. it is necessary to have a set of training samples for which the corresponding class is known. Then, the method is able to learn which sample corresponds to which class, and can assign new observations to classes after the training is complete. The observations are characterized using explanatory variables, referred to as features.

In myoelectric control the classes are intended movements, e. g. elbow flexion, hand open, etc., and the features are typically time- and frequency domain characteristics of the recorded EMG signal (Figure 2.4.3) (Hudgins, Parker, and Scott 1993).

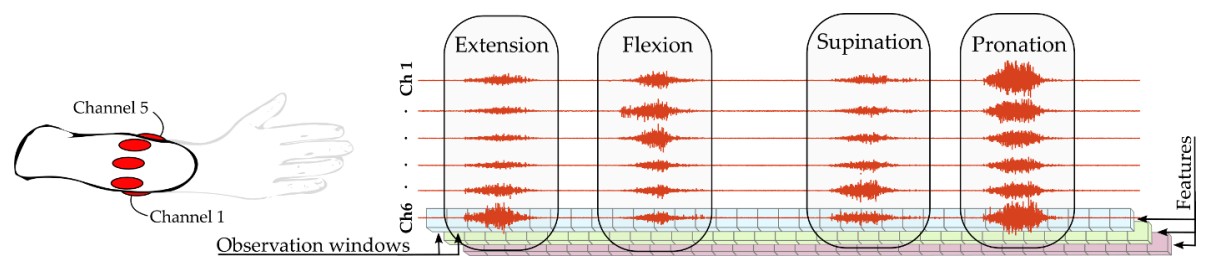

Figure 2.4.3: Conceptual depiction of pattern recognition methods. The required movement of the prosthesis is prompted by executing the intended movement with the phantom limb, leading to a more natural control. A machine learning method is trained to classify $E M G$ patterns belonging to the intended movements (classes). In this figure, the result of the classification is shown in the grey boxes. This method requires more electrodes than conventional methods, 6 electrodes placed symmetrically around the forearm in this example (red), and more sophisticated signal processing. This involves segmenting the signal into observation windows and calculating time- and frequency domain features for each window (illustrated with blue, green and pink features for channel 6). This picture also shows that the main drawback is that it is not possible to actuate more than one DoF (movement) simultaneously without extending the number of classes. 
Pattern recognition methods are capable to classify the user's intended movement with a greater than $95 \%$ accuracy, depending on the number of electrodes, on the number of estimated classes, and on the type of classifier (Chu, Moon, and Mun 2006; Englehart and Hudgins 2003; Li, Schultz, and Kuiken 2010). Pattern recognition outperforms conventional control in functional tests with the same tasks for both methods, if the task requires the use of more than one DoF (Hargrove, Lock, and Simon 2013; Smith et al. 2011; Young et al. 2014).

One disadvantage of pattern recognition is that it can only provide sequential control (Jiang et al. 2012b). Because one class is identified for each window, it is impossible in this paradigm to use more DoFs simultaneously.

Although it is possible to extend pattern recognition by training mixture classes to the classifier, e.g. simultaneous hand close and elbow flexion, this solution has several drawbacks. Firstly, the number of classes increases substantially, affecting classification accuracy (Ingraham et al. 2015; Young et al. 2013, 2014). Secondly, during simultaneous movements it is not possible to regulate the speed of the individual movements (Scheme et al. 2013; Simon et al. 2011).

As a result, pattern recognition cannot achieve a natural, intuitive control that would be similar to the control of our own hand (Jiang et al. 2012b).

\subsubsection{Regression}

Recently, regression methods were proposed to provide simultaneous and proportional control of multiple degrees of freedom (Jiang 2009). Instead of classifying the feature patterns, regression methods directly estimate kinematic variables that describe the intended movement, e. g. joint angles of the wrist. By directly estimating kinematics this approach is capable to reconstruct the natural movement of each DoF simultaneously and independently, provided the regression accuracy is high enough (Figure 2.4.4).

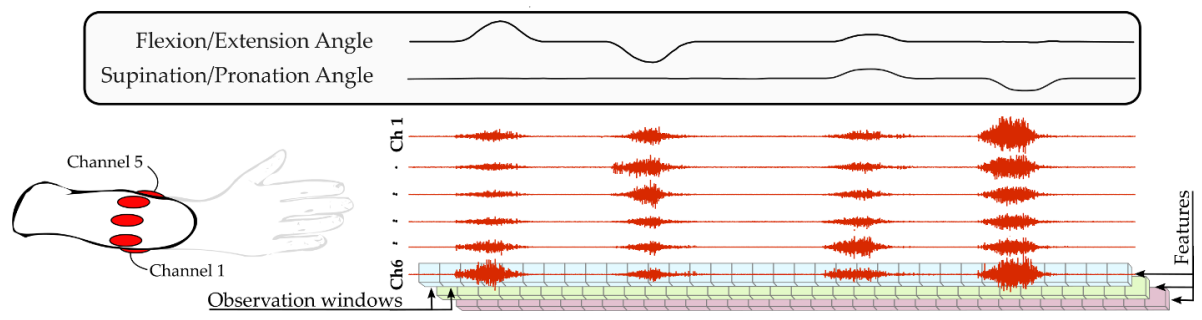

Figure 2.4.4: Conceptual depiction of regression methods. The prosthesis performs the intended movement by estimating movement kinematics based on the EMG (gray box). The joint angles in multiple DoFs are estimated simultaneously based on features (illustrated with blue, green and pink features for channel 6) of the EMG (red), resulting in smooth and natural movement. Note that simultaneous movements are also possible to estimate, and in general there is no restriction to the performed movement other than those imposed by mechanical constraints.

Regression methods use the same methodology as pattern recognition, in that they extract similar features from the EMG signal as a control input (Hahne et al. 2014; Nielsen et al. 2011). To estimate wrist kinematics based on these features, both supervised and 
unsupervised learning can be used, such as artificial neural networks (ANNs), linear regression, or non-negative matrix factorization (Hahne et al. 2014; Jiang 2009).

Numerous studies have reported successfully using regression techniques to achieve proportional and simultaneous control. In particular, Hahne et al. (2012) showed that it is possible to achieve simultaneous control without including simultaneous movements in the training data due to the generalization ability of estimators. Amsüss et al. (2015, 2016) showed that simultaneous and proportional control outperforms both pattern recognition and conventional control in clinically evaluated hand control tests on subjects with limb deficiency. These results suggest that regression methods are promising candidates for future prosthesis control approaches.

\subsection{Neural information in myoelectric control}

A severe limitation of all above prosthesis control methods is that amputees with transhumeral (above-elbow) or more proximal amputations do not have enough muscles for a myoelectric interface. They may be able to control the elbow of an arm prosthesis using the biceps and the triceps, but they lack the complete forearm and thus have no muscles to control the wrist and the hand. Patients with shoulder disarticulations have even less muscles to interface, yet they need the most functions replaced by the prosthesis. In such situations it would be advantageous to be able to interface a prosthetic device directly with motor nerves, obviating the need for residual muscles.

Intramuscular EMG recordings are capable to provide more direct information about neural activity than surface EMG, because the information about the source of the signal is inherently provided, without crosstalk from other sources (Basmajian and De Luca 1985). Indeed, by targeting muscles used to control of the wrist, it is possible to provide simultaneous and proportional myoelectric control that outperforms pattern recognition (Smith, Kuiken, and Hargrove 2014).

More invasive methods aim to interface a prosthetic device directly with motor nerves. There are three groups of existing peripheral nerve interfaces designed to control prosthetic devices (Navarro et al. 2005). Extraneural electrodes are placed around the nerves to record the activity of a population of neurons. These can take the form of electrode cuffs, books or flat electrodes (Rodri et al. 2000; Tyler and Durand 2002).

Conversely, intraneural electrodes penetrate the nerve to provide a greater selectivity. These can be implanted longitudinally or transversally with respect to the nerve fascicle, or penetrating it with a high density array of microelectrodes (Boretius et al. 2010; Lawrence et al. 2004; Navarro et al. 2005). Regenerative electrodes differ from both previous types in that they require to be implanted between severed ends of a nerve, and contain holes that the nerves can grow through during regeneration. Using the holes as electrodes, such interfaces are capable to record from small groups of motor units or even individual axons (Lago et al. 2005).

Although these technologies offer better selectivity and the potential for simultaneous sensory nerve stimulation and recording, there are still a number of challenges to over- 
come. These include chronic implantation and biocompatibility, data transfer between the electrode and the prosthesis, low signal to noise ratio, EMG interference or performing simultaneous recording and stimulation (Chu et al. 2012; Dhillon and Horch 2005; Gak et al. 2008). Even after solving these challenges, implanted devices will have the drawbacks of more difficult maintenance and the risk of infection, especially in the case of non-wireless interfaces (Micera et al. 2008; Tillander et al. 2010).

\subsection{Targeted muscle reinnervation}

A successful approach to resolve the problem of absent muscles not being able to contribute to a myoelectric interface is TMR (Kuiken et al. 2004). The basic concept of TMR is that we can use still available accessory muscles that have lost their function because of the amputation (such as m. pectoralis or m.latissimus dorsi) to amplify neural signals of amputated muscles (Figure 2.6.1). This is in accordance with the concept that in myoelectric interfacing muscles are essentially biological amplifiers of neural signals (Aszmann, Dietl, and Frey 2008).
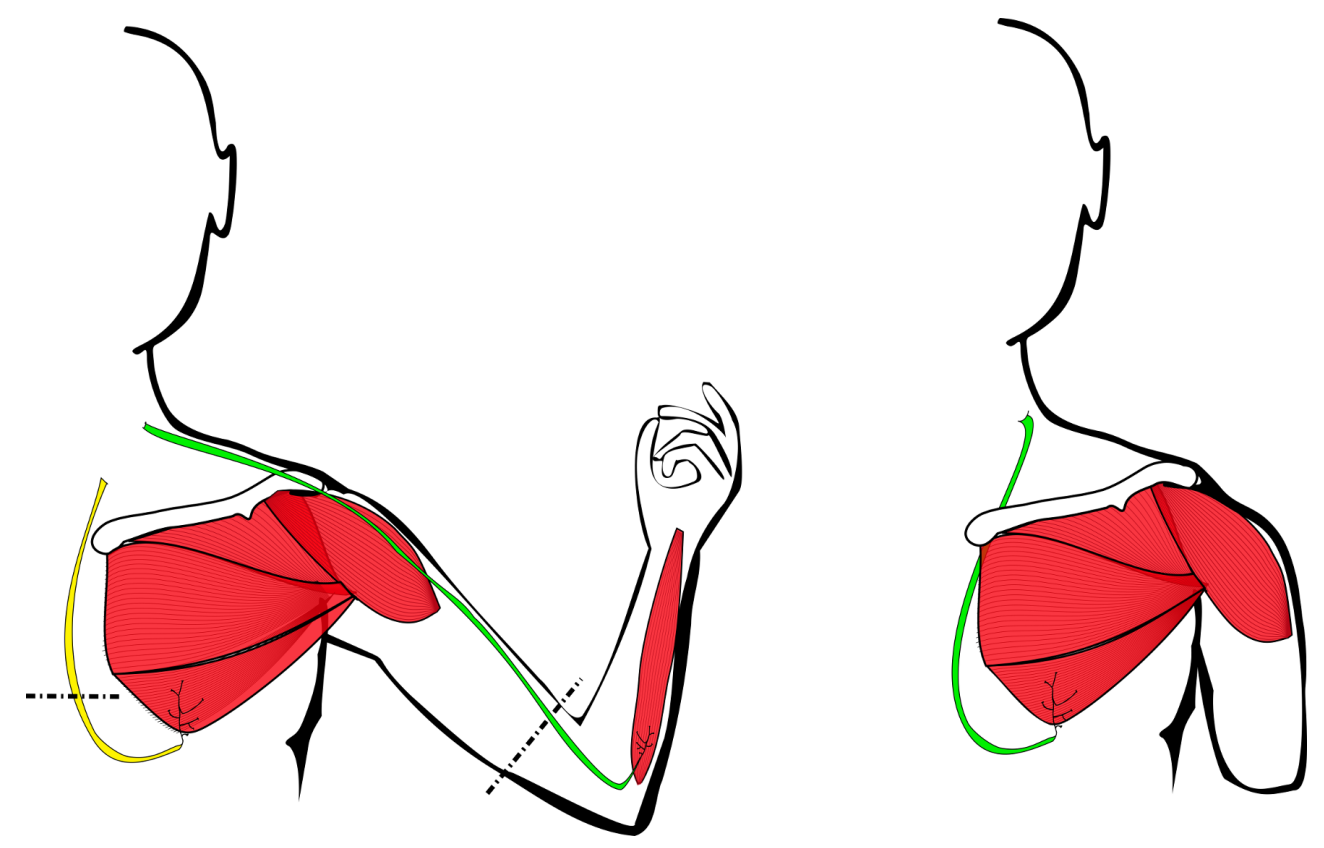

Figure 2.6.1: Illustration of the TMR procedure, before (left) and after the amputation (right). The nerve leading to a muscle in the forearm (green) is cut (dashed lines) and fused with a nerve leading to the $m$. pectoralis (yellow), which is reinnervated with the new (green) nerve. After the reinnervation, if the subject attempts to perform physiological tasks with the forearm, e.g. hand opening, a contraction in the chest can be observed.

To achieve this, nerves leading to these accessory muscles are cut and refused with nerves previously leading to now missing muscles. The transferred nerves then reinnervate the accessory muscles, resulting in muscles that contract whenever the central nervous system sends neural signals to the missing muscles. This technique also allows for several other measures that facilitate prosthesis control. Firstly, the reinnervation sites can be 
separated by targeting isolated muscle parts with different nerve branches, sometimes placing subcutaneous fat between the targeted muscle segments. Secondly, removing excess fat layer above the reinnervated muscles increases the performance of the myoelectric interface is also possible (Dumanian et al. 2009).

This technique have been demonstrated to enable the control of multiple-DoF devices for patients with high level amputations (Hargrove, Lock, and Simon 2013; Kuiken TA et al. 2009), to reverse cortical reorganization after an amputation, which also has been linked to phantom limb pain (Chen et al. 2013; Flor et al. 1995), and to also restore lost sensory information flow by sensory reinnervation (Hebert et al. 2014; Marasco, Schultz, and Kuiken 2009). Although it requires more extensive surgical treatment, it can be used with non-invasive surface interfacing, even using commercially available prostheses. Another advantage of this technique is that TMR patients can use the same motor commands as for the healthy hand to drive the prosthetic device.

Because there is a corresponding reinnervation site for each physiological movement, simultaneous and proportional control can also be achieved by using electrodes over each reinnervation site and the conventional control method described in Section 2.4.1. However, Hargrove, Lock, and Simon (2013) showed that conventional control might not be the best method to provide simultaneous and proportional control, since pattern recognition outperforms the industrial state-of-the-art even for TMR subjects. 


\section{THESIS CONTRIBUTION}

A common characteristic of myoelectric control methods reviewed in Section 2.4 is that they use time-frequency features as a means to extract information form the EMG. In this context the EMG is viewed as a stochastic signal (Nazarpour et al. 2013). The presence of MUAPs in the EMG signal, as described in Section 2.2, is therefore not directly exploited to determine the neural drive.

However, with recent advancements in EMG decomposition it has become feasible to extract direct neural information from the EMG signal automatically and non-invasively, as described in Section 2.3. Thus, instead of implicitly extracting control information through time-frequency analysis and machine learning, it would also be possible to implement a truly neural control, using the neural drive to the muscles to control a prosthetic device.

The estimation performance of regression methods could be greatly improved using this novel type of information. This is because the neural drive to the muscles could in principle be used in biomechanical models of the wrist to estimate muscle activity, muscle force, wrist dynamics and ultimately wrist kinematics. Such an approach could therefore take the physiological parameters and limitations of the wrist into account and produce natural kinematics in a variety of conditions, as opposed to current regression methods estimating wrist kinematics using the black box approach of machine learning.

The aim of this $\mathrm{PhD}$ project was to investigate whether a control framework using neural information could provide better control performance than conventional myoelectric control. To assess this, the following objectives were defined and addressed in individual studies:

- In the context of neural control TMR is especially significant, because in these patients the muscles used for prosthesis control are innervated by neurons that originally belonged to other muscles. Therefore I investigated whether the neural changes are reflected in electrophysiological characteristics, and how they affect prosthesis 
control. The results of this investigation are discussed in Study 1.

- As an initial evaluation of neural information in prosthesis control I investigated whether it improves the performance of the well-established pattern recognition method based on time domain features. I have found that neural features indeed outperform traditional features in this context, as discussed in Study 2.

- To translate the results of pattern recognition to regression, it was necessary to show that neural information can also be extracted from the surface EMG in nonisometric contractions characteristic for regression. This investigation was conducted in transradial amputees without TMR, to also establish whether advanced control is also possible without the distinct isolation of movement classes on the skin surface. The feasibility of EMG decomposition in non-isometric conditions and the properties of the extracted neural information regarding myoelectric control were assessed in Study 3.

- In the next step I developed a neural information based linear regression approach, demonstrating the feasibility of regression using neural information decomposed from the surface EMG. I validated the method in Study 4 by comparing its performance to regression using time-domain EMG features, in subjects with limb deficiency as well as healthy controls.

- In the final study I assessed whether enhancing neural information with physiological modeling improves control performance, exploiting the inherent connection of neural information with physiological variables. To this end I developed a nonlinear regression method using neural information and neuromusculoskeletal modeling and compared the performance of this method with state of the art nonlinear regression based on time-domain EMG features.

The above studies will be further discussed in detail in the following sections. 


\subsection{Study 1: Electrophysiological changes in motor units after targeted muscle reinnervation}

The results of this study are published in Kapelner et al. (2016) under the Creative Commons Attribution (CC BY) license, permission to reuse content was therefore not necessary.

Author contributions: conceived and designed the experiments, performed the experiments, analyzed the data, wrote the manuscript.

\subsubsection{Introduction}

As discussed in Section 2.6, TMR improves the capability of patients with high amputation levels to control myoelectric prostheses (Aszmann, Dietl, and Frey 2008; Dumanian et al. 2009; Kuiken TA et al. 2009; Marasco, Schultz, and Kuiken 2009). However, the physiology of the newly formed motor units after reinnervation is not well explored. The characteristics of these units could provide insights into the neurophysiological events following TMR, and might reveal important factors in the design of a novel control algorithm based on neural information.

The most important characteristic with respect to prosthesis control is the spatial MUAP distribution. This is because TMR aims to separate movement classes on the skin surface by connecting the corresponding nerve branches to separate muscle parts (Dumanian et al. 2009). Thus, if TMR is able to create perfect separation of the classes on the skin surface, neural information based methods would offer limited advantage over EMG features. Previous research suggests however, that the spatial separation might not be perfect (Hargrove, Lock, and Simon 2013; He Huang et al. 2008).

Due to the removal of subcutaneous fat over the muscle, the surface MUAP representations after TMR are expected to have smaller areas, shorter durations and larger amplitudes compared to healthy controls (Roeleveld et al. 1997). Because the innervation number of larger, force producing muscles are generally higher than those of muscles performing precise, fine-tuned movements, another possible consequence of the TMR procedure is the reduction of motor unit size, because the newly innervating motor neurons originally innervated smaller muscles (Enoka 2008).

This section will provide a general characterization of motor units in TMR patients compared with able-bodied controls by using non-invasive electrophysiological methods. The scope of the study is limited to motor units in the $m$. pectoralis major, which is easily accessible irrespective of the amputation profile.

\subsubsection{Methods}

\section{Subjects}

Five TMR patients underwent surgery at the Medical University of Vienna, and had full reinnervation of their targeted muscles before participating in this experiment (Table pectoralis). The detailed patient conditions and the experimental protocol were presented in 
Table 3.1: Age, gender and amputation details of TMR patients. Additional information on the TMR patients can be found in (Roberto Merletti and Dario Farina 2016). Only TMR sites of the $m$. pectoralis are listed. This table was published in Farina et al. (2014) and Kapelner et al. (2016).

\begin{tabular}{c||c|c|c|c|c|c|c|c}
\multirow{2}{*}{ Subject } & Age & Gender & $\begin{array}{c}\text { Amputation } \\
\text { details }\end{array}$ & $\begin{array}{c}\text { Time since } \\
\text { amputation }\end{array}$ & $\begin{array}{c}\text { Time since } \\
\text { TMR }\end{array}$ & $\begin{array}{c}\text { clavicular } \\
\text { head }\end{array}$ & $\begin{array}{c}\text { sternocostal } \\
\text { part }\end{array}$ & $\begin{array}{c}\text { abdominal } \\
\text { part }\end{array}$ \\
\hline \hline $\mathrm{T} 1$ & 25 & Male & $\begin{array}{c}\text { shoulder } \\
\text { disarticulation, } \\
\text { right }\end{array}$ & $\begin{array}{c}3 \text { years and } \\
2 \text { months }\end{array}$ & 10 months & $\begin{array}{c}\text { n. musculo- } \\
\text { cutaneous }\end{array}$ & n. medianus & n. medianus \\
\hline $\mathrm{T} 2$ & 32 & Male & $\begin{array}{c}\text { Glenohumeral, } \\
\text { left }\end{array}$ & $\begin{array}{c}3 \text { years and } \\
2 \text { months }\end{array}$ & 9 months & n. ulnaris & n. medianus & - \\
\hline $\mathrm{T} 3$ & 40 & Male & $\begin{array}{c}\text { shoulder } \\
\text { disarticulation, } \\
\text { left }\end{array}$ & $>5$ years & $\begin{array}{c}1 \text { year } 5 \\
\text { months }\end{array}$ & $\begin{array}{c}\text { n. musculo- } \\
\text { cutaneous }\end{array}$ & n. medianus & n. medianus \\
\hline $\mathrm{T} 4$ & 76 & Male & $\begin{array}{c}\text { Glenohumeral, } \\
\text { right }\end{array}$ & $>5$ years & $\begin{array}{c}11 \text { months } \\
\text { n. medianus }\end{array}$ & $\begin{array}{c}\text { n. medianus } \\
\text { n. cutantebrachii } \\
\text { medialis } \\
\text { (sensory) }\end{array}$ \\
\hline $\mathrm{T} 5$ & 11 & Female & $\begin{array}{c}\text { Glenohumeral, } \\
\text { left }\end{array}$ & $\begin{array}{c}2 \text { years } 3 \\
\text { months }\end{array}$ & $\begin{array}{c}1 \text { year } 5 \\
\text { months }\end{array}$ & $\begin{array}{c}\text { n. musculo- } \\
\text { cutaneous }\end{array}$ & n. medianus & n. medianus
\end{tabular}

(Farina et al. 2014). Nine male able-bodied subjects were included in the control group (mean age $30 \pm 5$ ); none of them had any neuromuscular disorders or abnormalities. All experimental protocols were designed and conducted according to the Declaration of Helsinki and approved by the local ethics committees. All participants signed written informed consent form prior to the experiment.

\section{Data acquisition}

For all recordings in this thesis high density multichannel surface EMG electrode grids were used (ELSCH064NM3, OTBioelettronica, Italy). These consisted of 64 electrodes in an 8 by 8 matrix connected by copper tracks on a kapton support, with an inter-electrode distance of $10 \mathrm{~mm}$ in both directions. They are applied on the skin using $1 \mathrm{~mm}$ thick two-sided adhesive foam, with holes corresponding to the electrode surfaces, which are filled with conductive paste to improve the skin-electrode contact. This procedure was the same for all subjects and all investigated muscles.

For this experiment the electrode grids were placed on the reinnervation sites of the $m$. pectoralis as described by the surgeon, for each subject individually (Figure 3.1.1). The electrode placement of the able-bodied subjects $\mathrm{H} 2-\mathrm{H} 4$ was equivalent with that of $\mathrm{T} 2$. To investigate the effect of electrode placement, subjects $\mathrm{H} 1$ and $\mathrm{H} 5-\mathrm{H} 9$ were equipped with electrodes on both sides, with electrode placements equivalent to that of T1 and T2 on the two sides.

The electrode grids were connected to a 256-channel EMG amplifier (EMGUSB2, OTBioelettronica, Italy), using a 64-channel impedance adapter with a gain of $5 \mathrm{~V} / \mathrm{V}$. All signals were recorded in monopolar mode, band pass filtered with cut off-frequencies of 3-500 Hz, and digitized with a 12 bit A/D converter at $2048 \mathrm{~Hz}$. The cut-off frequencies for able-bodied subjects were 10-900 Hz. 

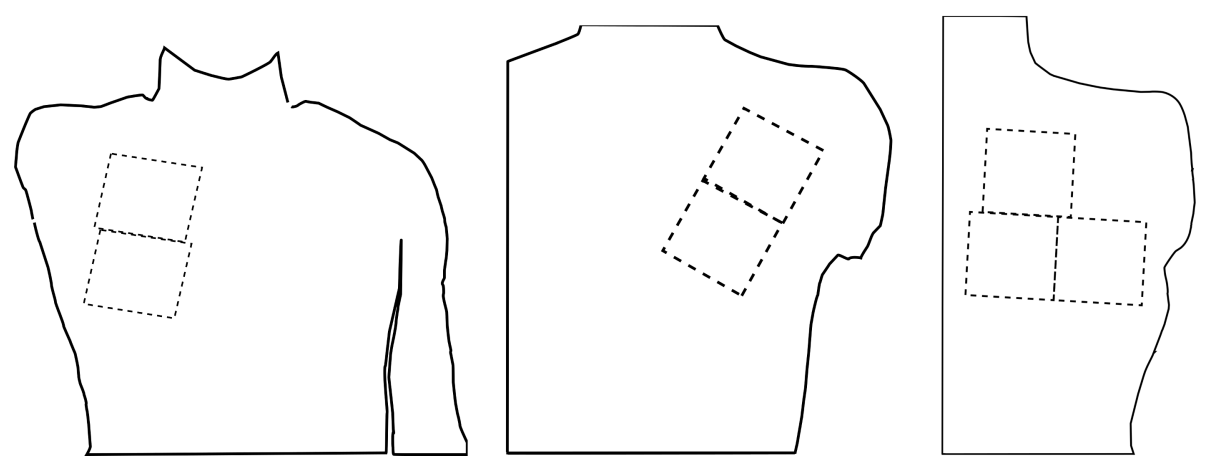

Figure 3.1.1: Electrode locations for the TMR patients: T1 and T4 (left), T2 and T5 (middle), T3 (right). The electrode placement of able-bodied subjects was the same as for T2 on the left side and as for T1 on the right side. This figure was published in Kapelner et al. (2016).

\section{Experiment protocol}

The TMR patients were instructed to perform the following tasks with the phantom limb in a randomized order: hand open, hand close, wrist extension, wrist flexion, thumb adduction, thumb abduction, thumb flexion, thumb extension, pronation, supination, elbow extension, elbow flexion. Not all subjects were able to perform all tasks. Patient T1 was able to sustain the contractions for $10 \mathrm{~s}$ with $5 \mathrm{~s}$ rest between attempts, the attempts of other TMR patients lasted $5 \mathrm{~s}$. T1, T4 and T5 performed two repetitions of 8 task attempts, T2 performed two task attempts twice and other four once (6 tasks in total), whereas T3 attempted two repetitions of 10 tasks. The tasks performance was guided by visual cues.

The able-bodied subjects attempted the following contractions with the $m$ pectoralis: sustained contraction at low force level, sustained contraction at medium force level, and a force ramp up from relaxation to medium force level followed by a ramp down. Force levels were not measured during the attempts and the subjects received no visual feedback about the force they were exerting, to provide similar conditions for both groups. Each attempt lasted 30 seconds, followed by rest for at least 5 seconds. Neither patients nor subjects reported fatigue.

\section{EMG Decomposition}

For patients T1, T2 and T3 the signals recorded from each grid were separately decomposed using the CKC algorithm described in Section 2.3. For all other subjects the two grids were jointly decomposed to increase the number of channels and thus decomposition efficiency.

\section{Motor unit action potentials and derived properties}

MUAP waveforms were estimated by multichannel spike-triggered averaging, as described in Section 2.3, using a 100 sample (48.83 ms) observation window centered on each spiking instant. Beforehand the EMG was high-pass filtered using a fourth order Butterworth filter with a cut-off frequency of $50 \mathrm{~Hz}$ to eliminate the ECG interference on the spike triggered 
averages.

The determination of motor unit size is not possible in vivo (Bodine-Fowler et al. 1990; Farina et al. 2008; Fuglevand et al. 1992). To quantify motor unit size changes after reinnervation indirectly, I used the change in the distribution of the electric potential on the skin surface, characterized by MUAP root mean square (RMS) maps (Figure 3.1.2). The map consisted of the RMS of each MUAP channel, defined as:

$$
R M S_{i}=\frac{1}{N} \sum_{n=0}^{N-1} x_{i}[n]^{2},
$$

where $\mathrm{N}$ is the length of the observation window, and $\mathrm{xi}$ is the spike triggered average of the i-th cahnnel. To gain sufficient resolution, the RMS maps were linearly interpolated to 10 samples per inter-electrode distance.

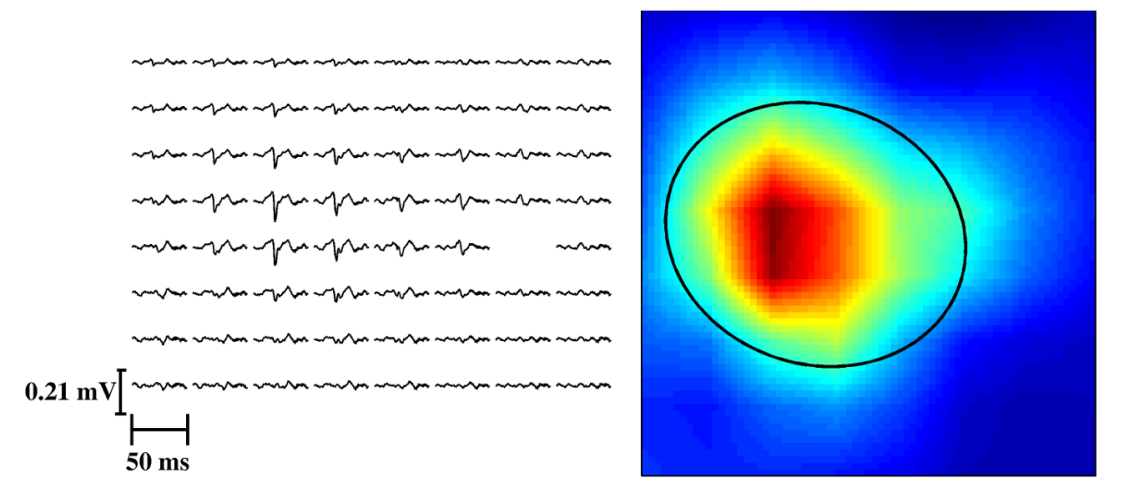

Figure 3.1.2: Multichannel MUAP shape of a decomposed motor unit of subject T3 (left) and the corresponding interpolated RMS map (right). One channel without a MUAP shape was excluded from the analysis because of signal artefacts (blank in the figure). The ellipse fitted on the RMS map of the motor unit is drawn in black on the right. Based on this fitting the motor unit in this example had a normalized MUAP surface area of 0.3. This figure was published in Kapelner et al. (2016).

Then, to each channel that had a greater RMS than $50 \%$ of the maximal RMS, an ellipse was fitted using a least squares fitting algorithm (Ohad Gal 2014). Unsuccessful fittings were repeated with manual correction. The areas of the fitted ellipses were normalized by the total area of one grid, and used as an indirect measure of motor unit size, referred to as normalized MUAP surface area in the rest of the section (Figure 3.1.2).

The durations of the action potentials were also calculated, defined as the time interval containing more than $80 \%$ of the action potential energy, centered on the maximal absolute value. Finally, maximal peak-to-peak amplitudes of the MUAPs were computed.

\section{Statistics}

For all the statistical comparisons between the two groups I used two-way nested analysis of variance (ANOVA) with an alpha level of 0.05. The random factor "Subject" with levels T1-T5, H1-H9 was nested in the fixed factor "Group", containing levels "TMR" and "Healthy". Only the left side of the able-bodied subjects was considered for this comparison, 
Table 3.2: Number of decomposed spike trains per task for each subject. Each column contains the number of spike trains for a given task (from Task 1 (M1) to Task 10 (M10)). Note that movements were different for each group. For the TMR patients movements were also different for each subject, because not all subjects were able to perform the same tasks with their phantom limb. Thus, each column in this table corresponds to a different task for each row, and the table only gives a general idea about the number of decomposed spike trains. This table was published in Kapelner et al. (2016).

\begin{tabular}{c|c|c|c|c|c|c|c|c|c|c|c}
\hline Subject & Side & M1 & M2 & M3 & M4 & M5 & M6 & M7 & M8 & M9 & M10 \\
\hline \hline T1 & Right & 2 & 5 & 3 & 2 & 6 & 15 & 4 & 7 & - & - \\
T2 & Left & 7 & 16 & 7 & 6 & 5 & 6 & - & - & - & - \\
T3 & Left & 9 & 2 & 8 & 4 & 7 & 9 & 5 & 0 & 6 & 10 \\
T4 & Right & 12 & 8 & 7 & 10 & 8 & 7 & 9 & 5 & - & - \\
T5 & Left & 7 & 5 & 7 & 10 & 7 & 5 & 6 & 6 & - & - \\
\hline H1 & Left & 19 & 6 & 0 & - & - & - & - & - & - & - \\
H1 & Right & 26 & 4 & 0 & - & - & - & - & - & - & - \\
H2 & Left & 3 & 8 & 2 & - & - & - & - & - & - & - \\
H3 & Left & 4 & 5 & 1 & - & - & - & - & - & - & - \\
H4 & Left & 5 & 4 & 7 & - & - & - & - & - & - & - \\
H5 & Left & 11 & 0 & 0 & - & - & - & - & - & - & - \\
H5 & Right & 9 & 0 & 0 & - & - & - & - & - & - & - \\
H6 & Left & 7 & 5 & 2 & - & - & - & - & - & - & - \\
H6 & Right & 1 & 2 & 2 & - & - & - & - & - & - & - \\
H7 & Left & 24 & 14 & 0 & - & - & - & - & - & - & - \\
H7 & Right & 12 & 12 & 0 & - & - & - & - & - & - & - \\
H8 & Left & 14 & 15 & 9 & - & - & - & - & - & - & - \\
H8 & Right & 20 & 15 & 5 & - & - & - & - & - & - & - \\
H9 & Left & 28 & 30 & 10 & - & - & - & - & - & - & - \\
H9 & Right & 31 & 21 & 5 & - & - & - & - & - & - & -
\end{tabular}

due to unavailable data for subjects $\mathrm{H} 2-\mathrm{H} 4$.

For comparisons between the two sides within the able bodied group the random factor "Subject" with levels H1, H5-H9 was nested in the fixed factor "Side", with levels "Left" and "Right".

The descriptive statistics used for reporting were mean and standard deviation.

\subsubsection{Results}

The EMG decomposition yielded 270 spike trains for the TMR patients and 398 for the able-bodied subjects (233 on the left and 165 on the right side, Table 3.2, Figure 3.1.3).

The normalized MUAP surface areas associated to the MU territories were significantly smaller for the TMR group $(p<0.001$, Figure 3.1.4). The mean normalized area was $0.25 \pm 0.17$ for TMR patients and $0.81 \pm 0.46$ for able-bodied subjects. MUAP durations of the TMR group were statistically significantly smaller $(10.92 \pm 3.89 \mathrm{~ms}$ and $14.03 \pm 3.91 \mathrm{~ms}$; $p<0.01$, Figure 3.1.4). MUAP peak-to-peak amplitudes were not significantly different between the two groups $(0.19 \pm 0.11 \mathrm{mV}$ and $0.14 \pm 0.06 \mathrm{mV} ; p=0.07$, Figure 3.1.4).

An analysis of the distribution of MUAPs over the surface covered by the electrode grids revealed that motor unit surface representations tended to group in the same regions and therefore overlapped with each other (Figure 3.1.5). The overall area on the electrode surfaces that contained motor units corresponding to one task only was on average 12.08 $\mathrm{cm}^{2}$, representing $11.8 \%$ of the total electrode surface, with a maximum of $18.9 \%$ (17.21 $\mathrm{cm}^{2}$ ) for subject $\mathrm{T} 4$. 


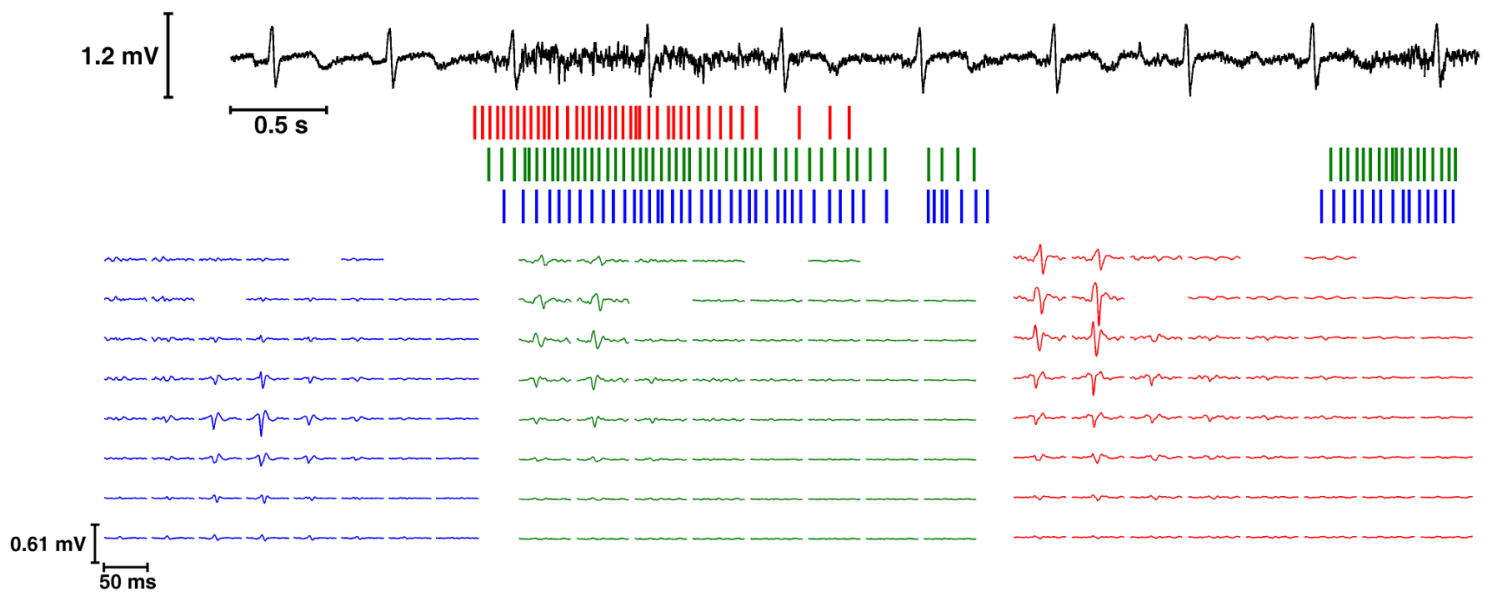

Figure 3.1.3: Representation of the decomposition of a single trial for subject T2. Above: one channel of the EMG signal. Below: bar plots of the decomposed spike trains and their MUAPs over the matrix. The colors of the spike trains and the MUAPs are matched. This figure was published in Kapelner et al. (2016).

There was no statistically significant difference between motor units in the left and right sides of able-bodied subjects, or any significant interactions between the factors Side and Subject. The mean values of the two groups were $0.76 \pm 0.43$ and $0.77 \pm 0.41$ for normalized MUAP surface area $(p=0.44), 13.72 \pm 3.52 \mathrm{~ms}$ and $13.30 \pm 3.80 \mathrm{~ms}$ for MUAP duration $(p=0.75)$ and $0.14 \pm 0.07 \mathrm{mV}$ and $0.13 \pm 0.06 \mathrm{mV}$ for MUAP amplitude $(p=0.48)$ for the left and right side of able-bodied subjects respectively.

\subsubsection{Discussion}

This section investigated the properties of motor units after TMR surgery in vivo based on surface EMG decomposition. The main findings were the significant difference of MUAP surface areas and durations between the two groups, and that surface areas were generally overlapping.

The first main finding was that MUAP surface areas in the $m$. pectoralis major of TMR patients were significantly smaller than those of able-bodied subjects. However, this difference does not necessarily indicate a difference in motor unit size. The indirect estimate of motor unit territory described in Methods is based on the MUAP, therefore it is also influenced by a number of factors, as described in Section 2.2, one of these being the volume conductor effect.

Because the subcutaneous fat layer over the targeted muscles is removed during the TMR procedure, the observed difference in MUAP surface areas might have resulted from TMR patients having thinner tissue layers between the muscle and the recording electrodes than able-bodied subjects. This is also indicated by the longer MUAP durations in the healthy group.

Another possible mechanism that results in smaller MUAP surface areas in TMR patients is that reinnervation may result in more compact fibre distribution than normal (Kugelberg, Edström, and Abbruzzese 1970). This was observed in self-reinnervated mus- 


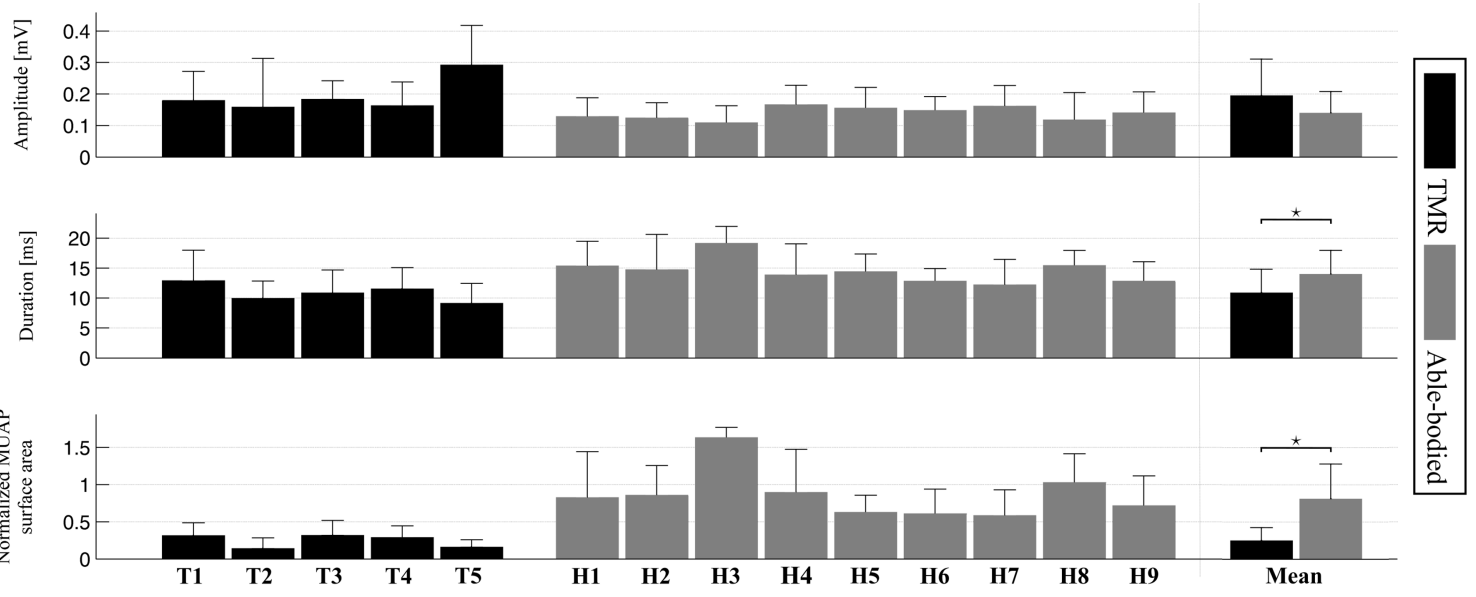

Figure 3.1.4: Mean and standard deviation of the investigated motor unit properties of the two groups. TMR patients are shown in black, able-bodied subjects in gray. The group means are shown on the right side. A part of this figure was published in Kapelner et al. (2016).
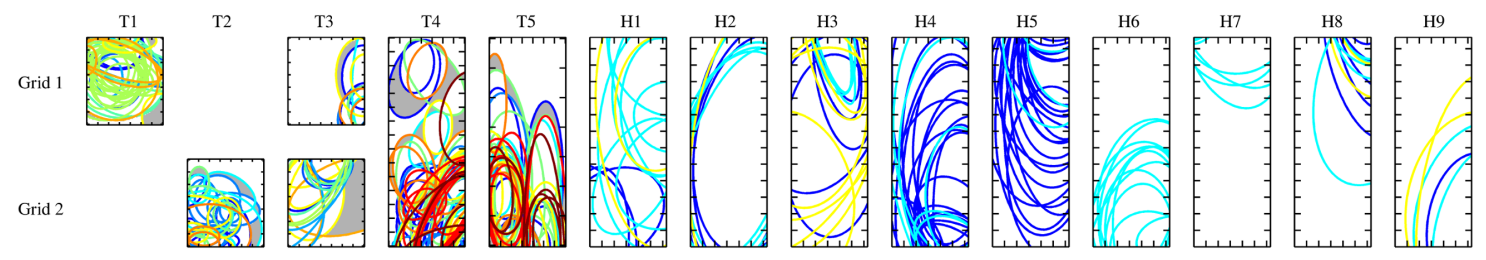

Grid 3

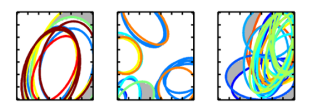

Figure 3.1.5: Spatial positions of the motor unit surface areas in each electrode grid (rows) for each subject (columns). Note that grid 1 for subject T2 and grid 2 for subject $T 1$ were not covering the $m$. pectoralis. Surface areas with the same colour in a given grid correspond to motor units identified in the same task. The area on the grid occupied by motor units active during only one task is coloured in grey. The tick marks on the image borders denote $1 \mathrm{~cm}$. This figure was published in Kapelner et al. (2016).

cles, whose cross-sectional size decreases due to an increased fibre density.

However, both thinner subcutaneous fat layer and increased fibre density in TMR patients would lead to increased MUAP amplitude. This was not observed in this experiment, and there was also no statistically significant difference between healthy motor unit properties with regards to sidedness, suggesting that the observed differences are unlikely to have resulted from differences in electrode placement. Thus, the most likely explanation for the findings is that motor units after reinnervation are effectively smaller in size, indicating that motor unit characteristics are influenced by properties of the new innervating nerve.

Changes in motor unit characteristics after reinnervation were documented in several cases (Buller, Eccles, and Eccles 1960). The most commonly mentioned such change is referred to as muscle plasticity, which incorporate changes in contractile and biochemical properties, changing the new muscle to resemble the original's properties more closely (Gordon and Pattullo 1993; Sreter, Luff, and Gergely 1975). Muscle fibre reorganization 
into dense regions was also observed, even in in human TMR patients (Kugelberg, Edström, and Abbruzzese 1970; Smith and Hargrove 2013). In some cases the muscle can also influence the innervating nerve, in so far as the success of the reinnervation depends on the capability of the muscle to adapt to the new neural input (Foehring, Sypert, and Munson 1987).

The results of this section suggest that some motor unit characteristics after reinnervation are indeed determined by the motor nerve. In particular, the smaller effective size of motor units after TMR may have resulted from the fact that the physiological motor unit sizes of the missing limb were smaller. However, it is also possible that the findings were a result of the aforementioned dense reorganization of the muscle units, or that both factors play a role in the resulting spatial localization. It was not feasible to directly test for these possible mechanisms in the in vivo setting of this study.

The other main finding of this section was the overlapping nature of MUAP surface areas. The spatial separation of EMG activity is one of the aims of the TMR procedure, as described in Section 2.6. This section showed that the EMG activity is spatially localized not only on a muscle-segment level, but also on a motor unit level. Although this spatial localization is created to facilitate direct prosthetic control, Figure XI suggests that MUAPs of motor units active in a given task overlap with MUAPs corresponding to other tasks. The large degree of overlapping is also shown by the largest area activated for a single motion class being only $18.9 \%$ of the total electrode grid surface. This finding suggests that motor units active in different tasks share similar territories in the muscle.

This might be the cause of difficulties in optimal electrode placement for maximizing class separation (He Huang et al. 2008), and it can also explain why more advanced control methods prove to be more effective for TMR patients than proportional control limited to a small number of spatially separable classes (Hargrove, Lock, and Simon 2013), (Huang et al. 2009).

This finding also demonstrates why a control approach based on direct neural information obtainable from surface EMG decomposition could lead to better performance: spatial separation is not necessary for surface EMG decomposition, because neural information based control is able to distinguish between movement classes even in the case of overlapping motor unit territories.

\subsubsection{Summary}

This section has shown that in terms of surface areas and action potential durations there are differences between reinnervated motor units and able-bodied controls. On the one hand, this may be due to physiological changes after TMR, such as the reduced fat layer or increased fiber density. On the other hand, it is also possible that the reinnervating motor neuron alters motor units characteristics.

The distribution of motor unit surface areas in TMR patients did not ensure highly separable EMG activities among different tasks, as indicated by the large degree of overlap of the surface areas. Although this conclusion is supported by previous research (Hargrove, 
Lock, and Simon 2013; Huang et al. 2009), a separate study would be needed to adequately analyse the separability of task for prosthesis control, and thereby evaluate the assumption that neural information might allow for better task separation. This investigation will be described in the next section. 


\subsection{Study 2: Pattern recognition based on neural information}

The results of this section are published in Kapelner et al. (2015) under IEEE copyright. In accordance with the requirements of the copyright holder, figures reused in this section are indicated by the copyright line O2015 IEEE, and the approval of the senior author, Prof. Dario Farina, is obtained to reuse a substantial portion of the original paper.

Author contributions: analyzed the data, wrote the manuscript.

The results of this section also constitute a part of the manuscript submitted for publication in Dario Farina et al. (2016). Figures 3.2.1 and 3.2.3 are based on figures of this publication, but were significantly changed and re-rendered.

Author contributions: performed the experiments, analyzed the data, revised the manuscript.

\subsubsection{Introduction}

The aim of this section is to assess the control capabilities of neural information as a feature for prosthesis control. To this end the well-established pattern recognition method will be used, comparing the classification accuracy of features based on neural information with that of traditional EMG features.

The comparison will be made in TMR subjects, in order to also evaluate the assumption of the previous section. This was that neural information might provide better separation of classes, because MUAP surface areas are distinguishable even if overlapping, as opposed to the EMG activity of different muscles that are influenced by crosstalk.

\subsubsection{Methods}

\section{Subjects}

Subjects T1, T2 and T3 from Section 3.1.2 participated in this experiment. For details on the amputation profiles and for subject description see Section 3.1.2 or (Farina et al. 2014). All experimental protocols were designed and conducted according to the Declaration of Helsinki and approved by the local ethics committee. All participants signed written informed consent form prior to the experiment.

\section{Data Acquisition and Experiment Protocol}

For data acquisition the same equipment and measurement protocol was used as described in Section 3.1.2. There was a difference in electrode placement, in that high-density surface electrode grids were placed above all reinnervation sites of the subjects, not just over the $m$. pectoralis, amounting to 6 grids per subject. For a detailed description of the electrode placement see Farina et al. (2014).

\section{EMG Processing}

To remove ECG artefacts the raw EMG signals were high-pass filtered with a cut-off frequency of $100 \mathrm{~Hz}$ using a 4TH order Butterworth filter, as suggested by Hargrove et 
al. (2009). As described in Section 2.4, the EMG features were calculated based on 100 ms observation windows with a $10 \mathrm{~ms}$ overlap between consecutive windows. Two sets of global features were compared. The first feature set included the RMS of all channels calculated by applying (3.1) to the EMG segments within each observation window. The other feature set included the RMS and the following time domain (TD) features, as proposed by (Hudgins, Parker, and Scott 1993):

- Waveform length: formally defined as $\sum_{n=1}^{N-1}|x[n]-x[n-1]|$, where $x$ is the EMG signal within an $N$ samples long observation window;

- Zero crossings: the number of instances within an observation window when $x[n] x[n-$ $1]<0$ and $x[n]-x[n-1]>d_{1}$, where $d_{1}$ is a subject specific pre-defined threshold;

- Slope sign changes: the number of such local extrema within an observation window, which exceed at least one neighboring sample in absolute value by a pre-defined threshold of $d_{2}$.

The feature space for both the RMS and the TD feature sets was reduced in dimension using Principal Component Analysis (PCA), retaining 9\% of the feature variance (Hotelling 1933; Person 1901).

\section{Spike Processing}

The recorded EMG signals were decomposed using the CKC algorithm. To ensure a fixed number of consistently detected spike trains for each trial, we pooled the decomposed spike trains into cumulative trains using two pooling methods:

- Grid pooling: each spike train decomposed from the EMG signals of a given electrode grid was assigned to the cumulative spike train of that grid. As a result, the feature dimension was 6 (one cumulative spike train per grid).

- Region pooling: The surface of each grid was further divided into four square regions, and one cumulative spike train was assigned to each region. The inclusion of spike trains to cumulative trains was based on the MUAP RMS map (Figure 3.2.1: a spike train was assigned to the cumulative train of all regions in which its respective MUAP contained significant activity, as determined by manual evaluation. As a result, the feature space had a dimensionality of 6 (number of grids) times 4 (number of regions per grid) $=24$.

For both of these approaches the feature was the number of spiking instances of the cumulative trains within the observation windows, using the same $100 \mathrm{~ms}$ intervals as for the RMS and TD feature sets.

\section{Classification}

The used classifier was a support vector machine (SVM) system with linear kernel, using the default C-value of the implementation (Chang and Lin 2011). SVM showed the best 


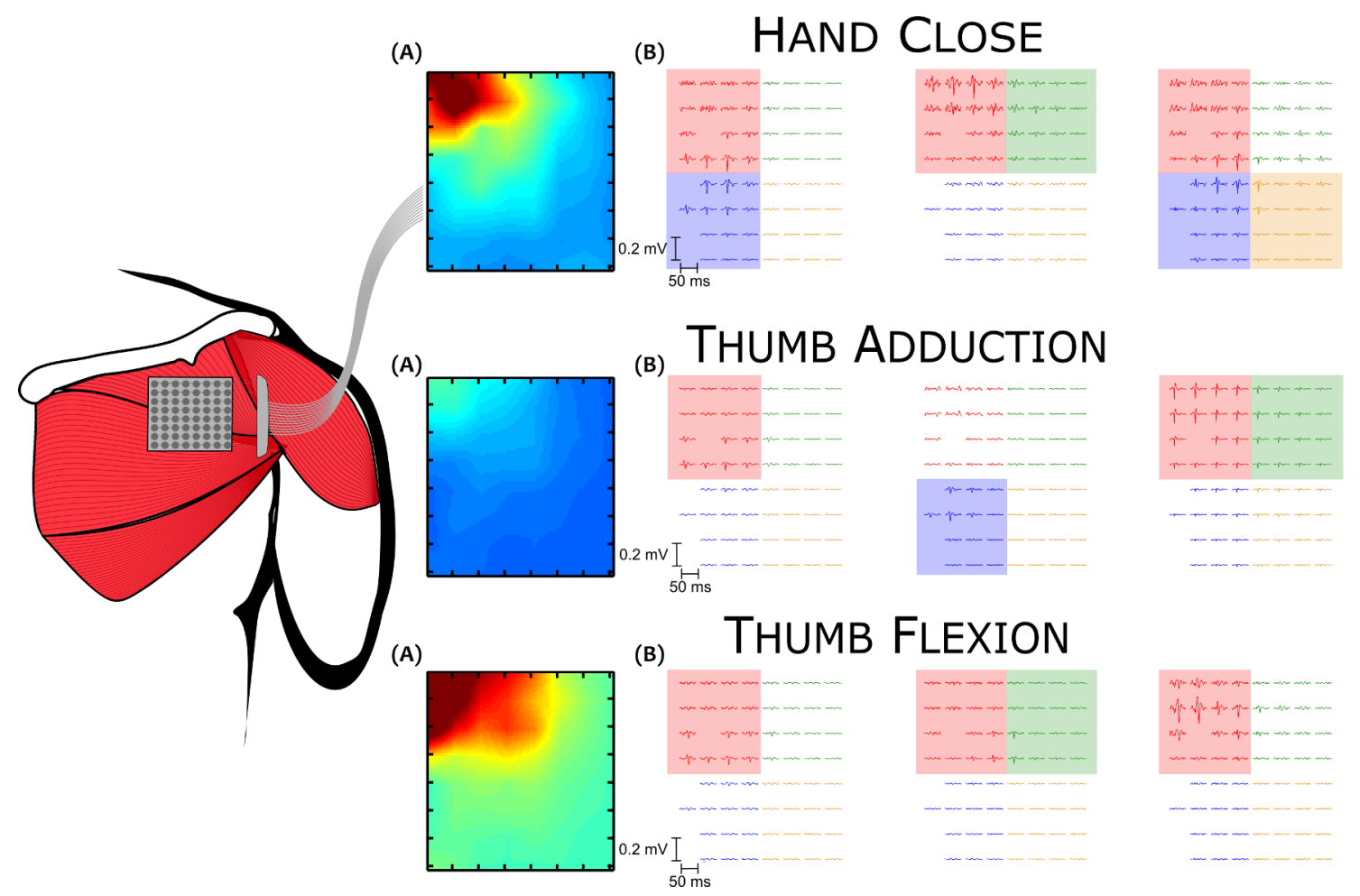

Figure 3.2.1: An illustration of region pooling for matrix 2 of subject T2. The mean RMS of all EMG channels during the performance of the task (A) and three MUAPs (B) are shown for three tasks (rows). The four cumulative spike trains extracted from the matrix are colour coded: spikes of motor units with MUAP activity in a given region (color) were pooled together. E.g. for hand close (upper row), spikes of the first motor unit were pooled into the red and blue cumulative trains, the second motor unit was assigned to the red and green trains, whereas spikes of the third unit were pooled into the red, blue and yellow cumulative spike trains, etc. Note that the three tasks are clearly separable using the color coded regions, whereas the EMG representations are highly similar.

overall classification accuracy out of several tested classifiers, while neither using other kernels nor individual C-value optimization improved the results significantly.

The assignment of movement classes (e.g., Hand Open) to samples was done based on the cue presented during the experiment. Moreover, this assignment was only done if at least one motor unit discharged one action potential in that sample, to avoid the inclusion of samples that contained no activity. This was required because most subjects were not regular prosthesis users, and had difficulties in sustaining contractions. In all remaining (unassigned) samples the subject was assumed to rest (No Movement class). The total number of classes was 9,7 and 11 for the three subjects.

For multiclass classification the one-against-one method was used with 3 sample majority voting as postprocessing (Englehart and Hudgins 2003). The classification results are provided with 5-fold cross-validation. An overview of the whole framework for both feature types is shown in Figure 3.2.2. 


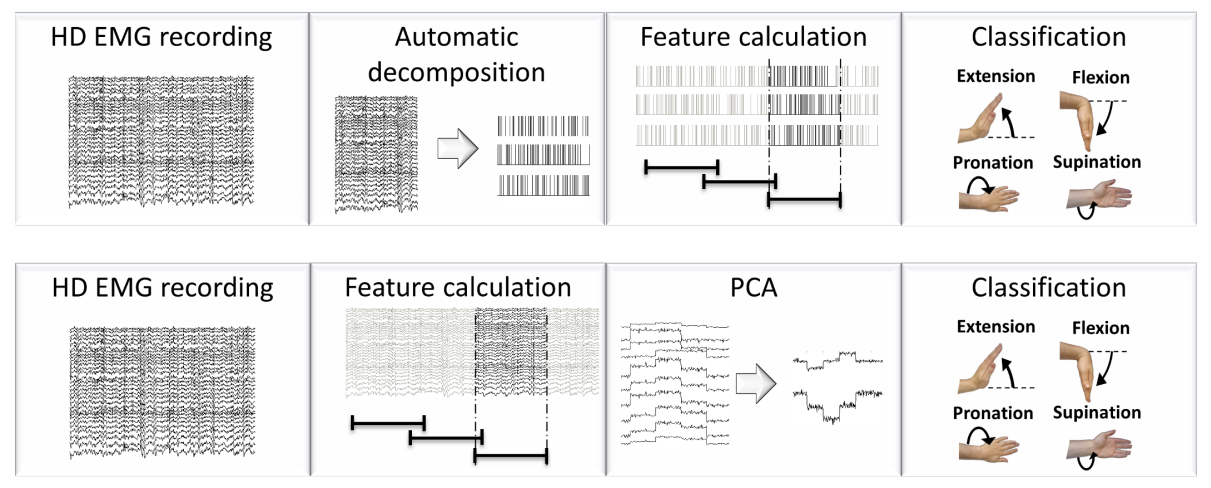

Figure 3.2.2: Block diagrams of the two compared pattern recognition approaches (О2015 IEEE). The difference between using neural information (top row) and EMG based features (bottom row) lies only in the signal processing blocks, the data acquisition and the classification method was the same for both feature types.

\subsubsection{Results}

Neural information with region pooling outperformed classic EMG features for all subjects (Figure 3.2.3). The most substantial increase in performance was observed for T3, who attempted 10 movements. Pooling all decomposed motor units of a given matrix together yielded lower classification accuracy than all other features except RMS. TD outperformed RMS for all subjects.

\subsubsection{Discussion}

The results showed that neural information contains sufficient information for pattern recognition in TMR to substantially outperform classic feature sets, even for a high number of classes.

In spite of the spatial separation and localization resulting from TMR, the lower classification accuracy of EMG based features shows that crosstalk between sites cannot be fully eliminated. The lower accuracy of grid pooling compared to region pooling and TD also shows that there were spike trains belonging to several classes within one grid. Pooling these together caused a decrease in class separability.

However, the additional spatial information introduced by region pooling was sufficient to increase classification accuracy substantially. Since the spatial EMG distribution after TMR is defined by the activity of specific muscles, the added information content of region pooling compared to grid pooling is likely related to function: pooling together spike trains leads to a decrease in control performance if the spike trains pooled together are not functionally similar.

It is compelling to argue that the reason for the superior performance of neural information is that it is more selective, because the spike trains belong to specific muscles and thus inherently contain functional information, regardless of discharge timings. However, intramuscular EMG, which also has the feature of each channel being muscle specific, does not outperform surface EMG (Smith and Hargrove 2013). This indicates that the increase 

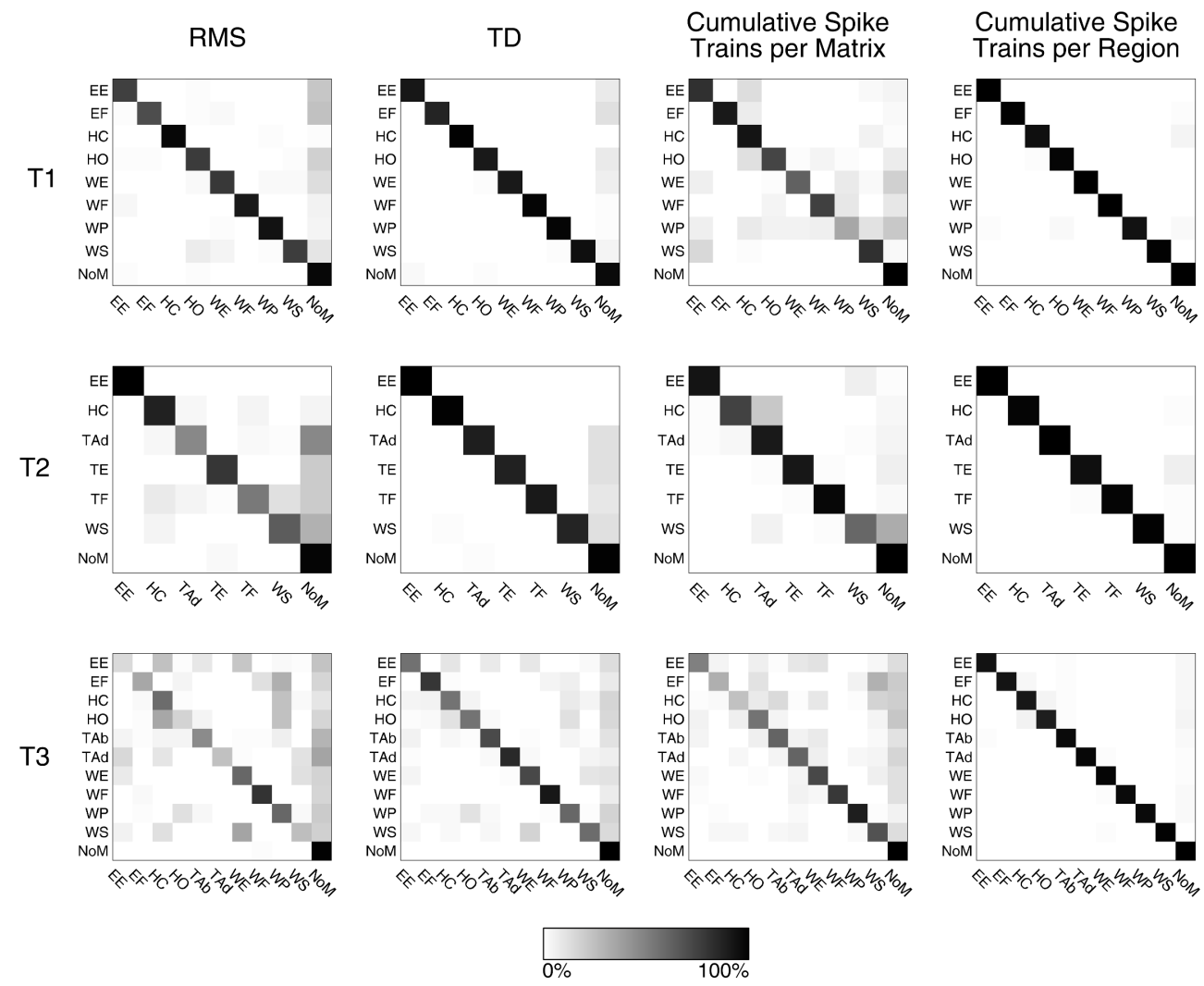

Figure 3.2.3: Confusion matrices for the classification of motor tasks. Each matrix contains classification accuracies from 0\% (white) to $100 \%$ (black). The rows of each matrix represent the performed tasks (classes), the columns the estimated (classified) tasks; e. g. the second column of the first row shows the number of cases (samples) that were classified as the second class, whereas the subject actually performed the first. The diagonals of the matrices represent all cases when the intended class was the same as the estimated (correct classifications). The classes were: elbow extension (EE), elbow flexion $(E F)$, hand close (HC), hand open (HO), wrist extension (WE), wrist flexion (WF), wrist supination (WS), wrist pronation (WP), thumb adduction (TAd), thumb abduction (TAb), thumb extension (TE), thumb flexion (TF) and no movement (NoM).

in performance is not only caused by neural information being more selective.

These findings also indicate that a full decomposition into spike trains distinctly belonging to specific motor units is not strictly necessary, as this section used only cumulative spike trains. Thus, an estimation of discharge timings of functionally similar units might also be sufficient for prosthetic control.

It is worth noting that TMR subjects can be trained to generate distinct EMG activations for each function, which could theoretically increase the classification accuracy of TD to the same level as observed using neural information (Englehart and Hudgins 2003; Hargrove, Lock, and Simon 2013). Nevertheless, the neural information based approach achieved an almost perfect separation of classes without prior training specific to this approach. This suggests that neural information based prosthesis control can provide a more natural control with less training, even if the increase in performance in a clinical scenario would not be this substantial. 


\subsubsection{Summary}

This section has confirmed the assumption made in the previous section, that in TMR patients neural information provides sufficient information to classify the intended movement more accurately than conventional EMG features do. The section also showed that while grouping together spike trains is necessary to ensure accurate classification, the resulting groups have to be functionally homogenous. This indicates that with an appropriate control algorithm motor unit spike trains could be used for advanced prosthesis control. 


\subsection{Study 3: The feasibility of EMG decomposition during voluntary contractions of the wrist}

The results of this study are submitted for publication in Kapelner, Negro, and Farina (2016). Upon acceptance the copyright will be transferred to IEEE. In accordance with the requirements of the copyright holder, figures reused in this section are indicated by the copyright line (1) 2016 IEEE, and the approval of the senior author, Prof. Dario Farina, is obtained to reuse a substantial portion of the original paper. Parts of Figure 3.3.1 also appeared in Kapelner, Jiang, and Farina (2016), permission for reuse was not required due to retained rights of the authors for inclusion in doctoral dissertations (see Section 3.4).

Author contributions: performed the experiments, analyzed the data, wrote the manuscript.

\subsubsection{Introduction}

The previous sections showed that neural information is indeed a promising alternative to traditional EMG based features when using established control methods in TMR, such as pattern recognition. The feasibility of EMG decomposition in TMR was established by Farina et al. (2014), but whether current decomposition methods are suitable to be used for advanced control purposes is not self-evident.

This is because - as discussed in Section 2.3 - current EMG decomposition methods were developed for isometric contractions at constant or slow-varying force, therefore their applicability in the case of voluntary contractions has to be determined, especially in the case of subjects with limb deficiency. Investigating this question is the first step in providing a proof of concept for an advanced prosthesis control method based on neural information. Another addressed question is whether advanced control is also possible in the case of transradial amputees without TMR, who lack distinct isolation of movement classes on the skin surface.

In this section the feasibility of EMG decomposition will be investigated in voluntary contractions that are characteristic to advanced prosthesis control using regression, for both able bodied subjects and patients with limb deficiency.

\subsubsection{Methods}

\section{Subjects}

Seven able-bodied subjects (A1-A7, age $29 \pm 6$ years) and two patients with limb deficiency (D1 and D2, age 43 and 57 years) participated in this experiment. All participants signed an informed consent form approved by the local ethics committee. None of the able-bodied volunteers had any neuromuscular abnormalities or disorders. D1 had a left side transradial amputation 37 years before this experiment; D2 had a transradial congenital amputation on the right side. Both patients were daily myoelectric prosthesis users. 


\section{Data Acquisition}

The EMG recordings were made using the high-density electrode grids described in Section 3.1. The grids were applied around the proximal third of the forearm of the dominant or amputated arm of the subjects (Figure 3.3.1/A). Two grids were mounted on subjects A4, A6, D1 and D2, while the other subjects had sufficient space on the forearm for three grids. Data acquisition was performed as described in Section 3.1.2, with the band-pass cut-off frequencies set to 3 and $900 \mathrm{~Hz}$.
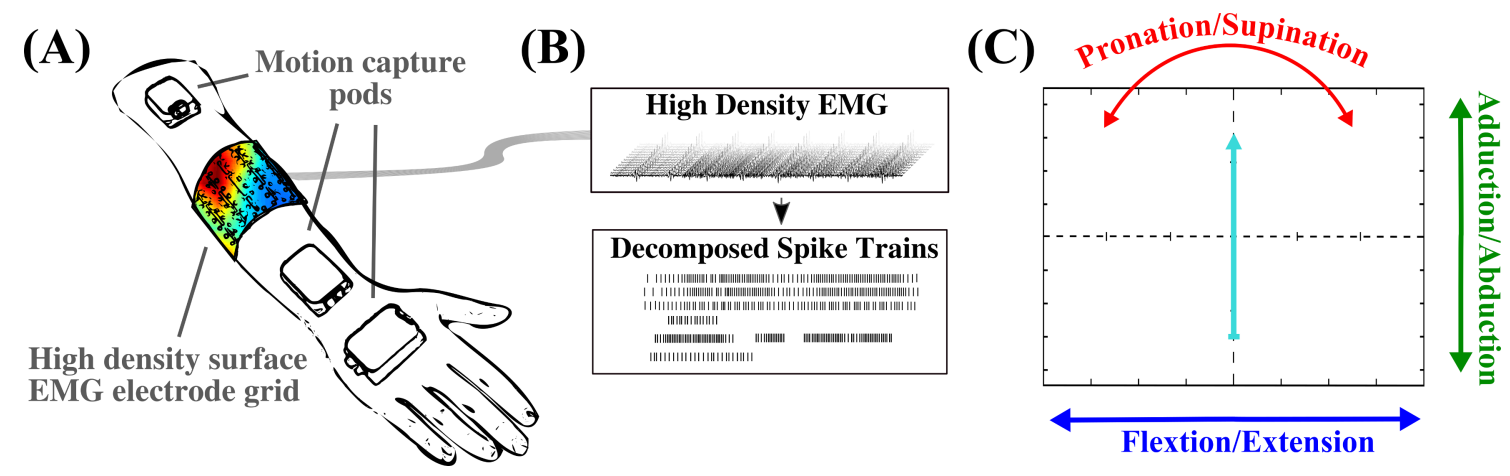

Figure 3.3.1: An illustration of the EMG recording and motion capture system (A), as well as the signal processing steps (B) and the visual feedback provided for the subjects $(C)$. As shown in $(C)$, subjects were presented an arrow as an indication of their wrist position and movements. Horizontal movement of the arrow corresponded to flexion and extension, vertical movement to radial and ulnar deviation, while the rotation of the arrow corresponded to pronation and supination. This figure was adapted from Kapelner, Jiang, and Farina (2016) and Kapelner, Negro, and Farina (2016).

To measure wrist kinematics during the contractions and to guide subjects' movements by visual feedback, three motion capture sensors (MTx, Xsens) were mounted on the following landmarks on the dominant arm: the dorsal side of the palm, the dorsal side of the wrist, and the dorsal side of the distal end of the upper arm. For subjects with limb deficiency, the sensors were placed on the contralateral (healthy) arm.

\section{Experiment Protocol}

The subjects were seated comfortably before the recording session, with the arm fully relaxed and the hand capable to move in the whole range of motion in all directions without obstacles. After a familiarization session the subjects were instructed to follow an arrow shaped visual cue, while the movement of their wrist was visualized by another arrow with a different color, as recorded by the motion capture sensors (Figure 3.3.1/C).

The movement of the cue prompted wrist movements in one DoF at a time from rest until the maximal range of movement, within $2.5 \mathrm{~s}$, then back to the rest position. This resulted in a triangular joint angle profile for each of the following motor tasks: flexion and extension corresponding to horizontal arrow movements, ulnar and radial deviation corresponding to vertical arrow movements, and pronation and supination corresponding to arrow rotation (Figure 3.3.1/C). All motor tasks were repeated three times in a randomized order. Patients with limb deficiency were instructed to perform mirrored bilateral 
movements, i. e. to match the movement of the phantom limb to the contralateral side, which was motion captured and used for kinematics measurement and feedback (Nielsen et al. 2011).

\section{EMG decomposition}

The EMG signals corresponding to each DoF were separately decomposed using a convolutive blind source separation method related to CKC (Negro et al. 2016). This method incorporates the calculation of an estimate for the accuracy of the decomposition, referred to as the silhouette measure (SIL) (Negro et al. 2016). Previous validation of SIL using concurrent intramuscular and surface EMG recordings showed that SIL values are linearly related to the rate of agreement with intramuscular EMG signals. Decomposed spike trains with an SIL greater than 0.8 were included in the subsequent analysis, corresponding to an approximate accuracy of at least $80 \%$. Spike trains having less than five discharges in all repetitions were disregarded.

The MUAP shapes as calculated by spike triggered averaging were used to compare motor units identified in different trials and match double occurrences. Motor units across more trials were considered to be the same if the maximal value of the normalized crosscorrelation function of their MUAP shapes was greater than 0.8. MUAP RMS maps were also calculated, as described in Section 3.1.2.

As an additional measure of decomposition performance the signal-to-interference ratio (SIR) was calculated, which shows the amount of the variance of the EMG signal explained by the identified spike trains (Hamid R. Marateb et al. 2011). To this end the MUAP trains were reconstructed by replacing each spiking instance in each spike train by the corresponding multichannel MUAP shape. The sum of all MUAP trains was then used as a reconstruction of the global EMG, and the SIR was calculated using the following formula:

$$
S I R_{i}=\frac{\sum_{n=0}^{N-1}\left(x_{i}(n)-z_{i}(n)\right)^{2}}{\sum_{n=0}^{N-1}\left(x_{i}(n)\right)^{2}} \cdot 100 \%,
$$

where $x_{i}(n)$ is the $n$-th sample of the $i$-th channel of the multichannel differential EMG signal, $z_{i}$ is $i$-th channel of the reconstructed EMG as described above, and $N$ is the number of recorded samples. This calculation was repeated for each repetition of each trial, including only the channels for which the reconstructed signal $z_{i}$ had an RMS greater than $20 \%$ of the maximal RMS for that repetition.

\section{Data Analysis}

I assigned the decomposed spike trains to motor tasks for each DoF separately, in accordance with the decomposition. A spike train was assigned to a task if $90 \%$ of its spikes were detected within the beginning and end times of the task, defined manually based on the recorded wrist kinematics for simplicity. 
The most relevant spike trains for prosthesis control are only active during one task, since the presence of such a train would allow the control method to directly identify the performed task. These units reside in the agonist muscles of a given task, and will be referred to as discriminative agonist units in the following.

A motor neuron was labeled as an agonist if $90 \%$ of its spikes were detected either during a given motor task or during the second half of the opposite (antagonist) task. The time intervals for the opposite task were defined as the time between reaching the maximal angle and the end time of that task.

It is worth noting that a motor unit could be classified as agonist for more than one DoF, e. g. motor units in the $m$. flexor carpi radialis act as agonist for both flexion and radial deviation. Therefore only agonists detected only in one DoF were categorized as discriminative agonist units.

Spike trains were smoothed using a $400 \mathrm{~ms}$ Hanning window, and the extrema of the discharge rates were calculated, as well as the absolute joint angles at recruitment normalized to the full range of motion (De Luca and Erim 1994). Additionally the number of agonist units detected consistently in all repetitions was counted for each motor task.

Means and standard deviations are reported for all calculated characteristics.

\subsubsection{Results}

The decomposition yielded $16 \pm 7$ spike trains per motor task (Figure 3.3.2) with a mean SIR value of $24.7 \pm 12.7 \%$. The average SIL was $0.86 \pm 0.05$. Out of $12 \pm 6$ agonist spike trains $6 \pm 5$ were present consistently.

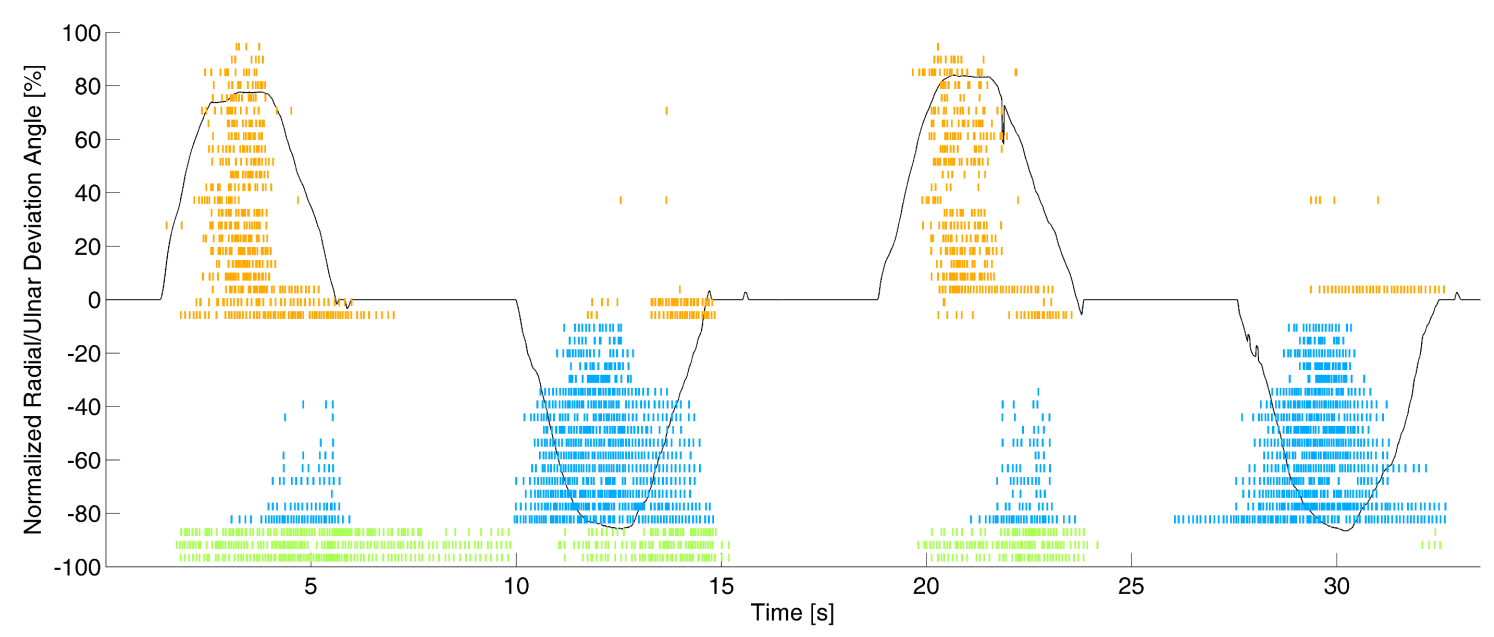

Figure 3.3.2: A representative example of the results of EMG decomposition (О2016 IEEE). Decomposed spike trains are colored according to their classification: orange spike trains belong to agonists for radial deviation, blue spike trains to agonists of ulnar deviation, and green spike trains did not belong to agonists for any motor task. Note that not all spike trains are present for each repetition of the tasks, and some are also present at rest. For the latter spike trains the angle at recruitment was 0, whereas the other spike trains generally corresponded to recruitment at greater angles, with only a few active motor units for angles smaller than $20 \%$ of the range of movement. This figure also appeared in Kapelner, Negro, and Farina (2016). 
For subjects A3 and A5 at least one discriminative unit was present in all repetitions of each motor task, and subject A2 had three such tasks. The remaining 6 subjects (including D1 and D2) had discriminative motor units consistently present in five motor tasks. The number of spike trains decomposed per task, the discharge statistics and the angles at recruitment are summarized in Tables 3.3 and 3.4.

Table 3.3: Number of decomposed spike trains per subject and discharge statistics for the able-bodied subjects (О2016 IEEE). The discharge statistics were calculated for the spike trains of all able-bodied subjects pooled together. Rows indicate which motor task the spike train belong to, or how many tasks they belong to, i. e. "One Motor Task" means that spike trains were only active during one task. Thus, based on this latter group a myoelectric control method could discriminate between motor tasks. Only spike trains belonging to agonists were included in those present in all repetitions. This table also appeared in Kapelner, Negro, and Farina (2016).

\begin{tabular}{|c|c|c|c|c|c|c|c|}
\hline & \multicolumn{3}{|c|}{$\begin{array}{l}\text { Number of decomposed spike } \\
\text { trains per subject }\end{array}$} & \multirow{2}{*}{$\begin{array}{l}\text { Minimal } \\
\text { discharge } \\
\text { rate [pps] }\end{array}$} & \multirow{2}{*}{$\begin{array}{l}\text { Maximal } \\
\text { discharge } \\
\text { rate [pps] }\end{array}$} & \multirow{2}{*}{$\begin{array}{c}\text { Normalized } \\
\text { angle at } \\
\text { recruitment [\%] }\end{array}$} & \multirow{2}{*}{ SIL } \\
\hline & Total & Agonists & $\begin{array}{l}\text { Present in all } \\
\text { repetitions }\end{array}$ & & & & \\
\hline Extension & $12 \pm 6$ & $10 \pm 5$ & $5 \pm 4$ & $6.72 \pm 2.68$ & $22.68 \pm 6.97$ & $59.81 \pm 26.28$ & $0.86 \pm 0.05$ \\
\hline Flexion & $16 \pm 8$ & $14 \pm 5$ & $5 \pm 4$ & $7.04 \pm 3.11$ & $22.68 \pm 6.24$ & $59.80 \pm 28.59$ & $0.86 \pm 0.05$ \\
\hline Radial Deviation & $18 \pm 6$ & $13 \pm 5$ & $7 \pm 6$ & $6.96 \pm 2.72$ & $22.96 \pm 6.62$ & $65.31 \pm 27.23$ & $0.85 \pm 0.04$ \\
\hline Ulnar Deviation & $21 \pm 6$ & $16 \pm 5$ & $11 \pm 5$ & $6.95 \pm 2.57$ & $22.18 \pm 6.21$ & $58.75 \pm 27.63$ & $0.87 \pm 0.05$ \\
\hline Pronation & $15 \pm 6$ & $9 \pm 5$ & $4 \pm 4$ & $6.34 \pm 2.88$ & $21.31 \pm 6.78$ & $58.67 \pm 29.76$ & $0.86 \pm 0.04$ \\
\hline Supination & $17 \pm 9$ & $11 \pm 8$ & $6 \pm 6$ & $6.46 \pm 2.17$ & $17.95 \pm 7.37$ & $58.10 \pm 27.14$ & $0.88 \pm 0.05$ \\
\hline $\begin{array}{c}\text { One Motor Task } \\
\text { (discriminative) }\end{array}$ & 6 & 62 & 17 & 6. & 09 & 66.04 & $0.86 \pm 0.05$ \\
\hline Two Motor Tasks & $14 \pm 8$ & $6 \pm 5$ & $3 \pm 3$ & $6.24 \pm 2.70$ & $19.01 \pm 5.51$ & $50.21 \pm 28.86$ & $0.87 \pm 0.05$ \\
\hline Three Motor Tasks & 0 & 0 & 0 & $6.42 \pm 2.10$ & $23.06 \pm 3.50$ & $21.71 \pm 15.11$ & $0.86 \pm 0.04$ \\
\hline Four Motor Tasks & $1 \pm 2$ & 0 & 0 & $6.14 \pm 1.92$ & $19.27 \pm 4.80$ & $44.69 \pm 17.94$ & $0.85 \pm 0.03$ \\
\hline Five Motor Tasks & 0 & 0 & 0 & $7.54 \pm 4.52$ & $27.90 \pm 2.02$ & $21.91 \pm 0.77$ & $0.83 \pm 0.03$ \\
\hline Six Motor Tasks & $0 \pm 1$ & 0 & 0 & $6.88 \pm 2.12$ & $24.54 \pm 2.82$ & $22.32 \pm 0.69$ & $0.91 \pm 0.02$ \\
\hline
\end{tabular}

Table 3.4: Number of decomposed spike trains per subject and discharge statistics for the subjects with limb-deficiency (О2016 IEEE). The discharge statistics were calculated for the spike trains of all subjects with limb-deficiency pooled together. Rows indicate which motor task the spike train belong to, or how many tasks they belong to, $i$. e. "One Motor Task" means that spike trains were only active during one task. Thus, based on this latter group a myoelectric control method could discriminate between motor tasks. In this group there were no spike trains present during more than two tasks. . Only spike trains belonging to agonists were included in those present in all repetitions. This table also appeared in Kapelner, Negro, and Farina (2016).

\begin{tabular}{c||c|c|c|c|c|c|c}
\multicolumn{1}{|c||}{} & \multicolumn{2}{|c|}{$\begin{array}{c}\text { Number of decomposed spike } \\
\text { trains per subject }\end{array}$} & $\begin{array}{c}\text { Minimal } \\
\text { discharge } \\
\text { rate [pps] }\end{array}$ & $\begin{array}{c}\text { Maximal } \\
\text { discharge } \\
\text { rate [pps] }\end{array}$ & $\begin{array}{c}\text { Normalized } \\
\text { angle at } \\
\text { recruitment [\%] }\end{array}$ & SIL \\
\cline { 2 - 4 } & Total & Agonists & $\begin{array}{c}\text { Present in all } \\
\text { repetitions }\end{array}$ & & & & \\
\hline \hline Extension & $15 \pm 12$ & $14 \pm 11$ & $6 \pm 3$ & $7.00 \pm 2.55$ & $22.92 \pm 8.35$ & $61.37 \pm 19.43$ & $0.84 \pm 0.04$ \\
\hline Flexion & $9 \pm 9$ & $8 \pm 8$ & $4 \pm 3$ & $6.58 \pm 3.17$ & $21.80 \pm 9.27$ & $57.81 \pm 25.71$ & $0.86 \pm 0.04$ \\
\hline Radial Deviation & $10 \pm 3$ & $9 \pm 1$ & $3 \pm 4$ & $6.45 \pm 2.34$ & $23.69 \pm 10.88$ & $71.37 \pm 17.00$ & $0.84 \pm 0.04$ \\
\hline Ulnar Deviation & $17 \pm 2$ & $16 \pm 1$ & $8 \pm 8$ & $7.57 \pm 2.01$ & $21.61 \pm 6.42$ & $58.07 \pm 23.96$ & $0.85 \pm 0.04$ \\
\hline Pronation & $13 \pm 6$ & $11 \pm 4$ & $5 \pm 2$ & $6.15 \pm 2.64$ & $19.19 \pm 5.84$ & $59.66 \pm 13.92$ & $0.87 \pm 0.05$ \\
\hline Supination & $15 \pm 19$ & $13 \pm 16$ & $5 \pm 7$ & $6.50 \pm 3.10$ & $20.51 \pm 9.11$ & $77.36 \pm 19.67$ & $0.86 \pm 0.05$ \\
\hline \hline $\begin{array}{c}\text { One Motor Task } \\
\text { (discriminative) }\end{array}$ & $15 \pm 12$ & $14 \pm 11$ & $6 \pm 3$ & $7.00 \pm 2.55$ & $22.92 \pm 8.35$ & $61.37 \pm 19.43$ & $0.84 \pm 0.04$ \\
\hline Two Motor Tasks & $9 \pm 9$ & $8 \pm 8$ & $4 \pm 3$ & $6.58 \pm 3.17$ & $21.80 \pm 9.27$ & $57.81 \pm 25.71$ & $0.86 \pm 0.04$
\end{tabular}

To obtain a surface representation of a given motor task, the MUAP surface maps of agonist motor units belonging to a given task were summed (Figure 3.3.3).

The mean absolute normalized angle at recruitment was $62.48 \pm 26.44 \%$ (Tables 3.3 and 


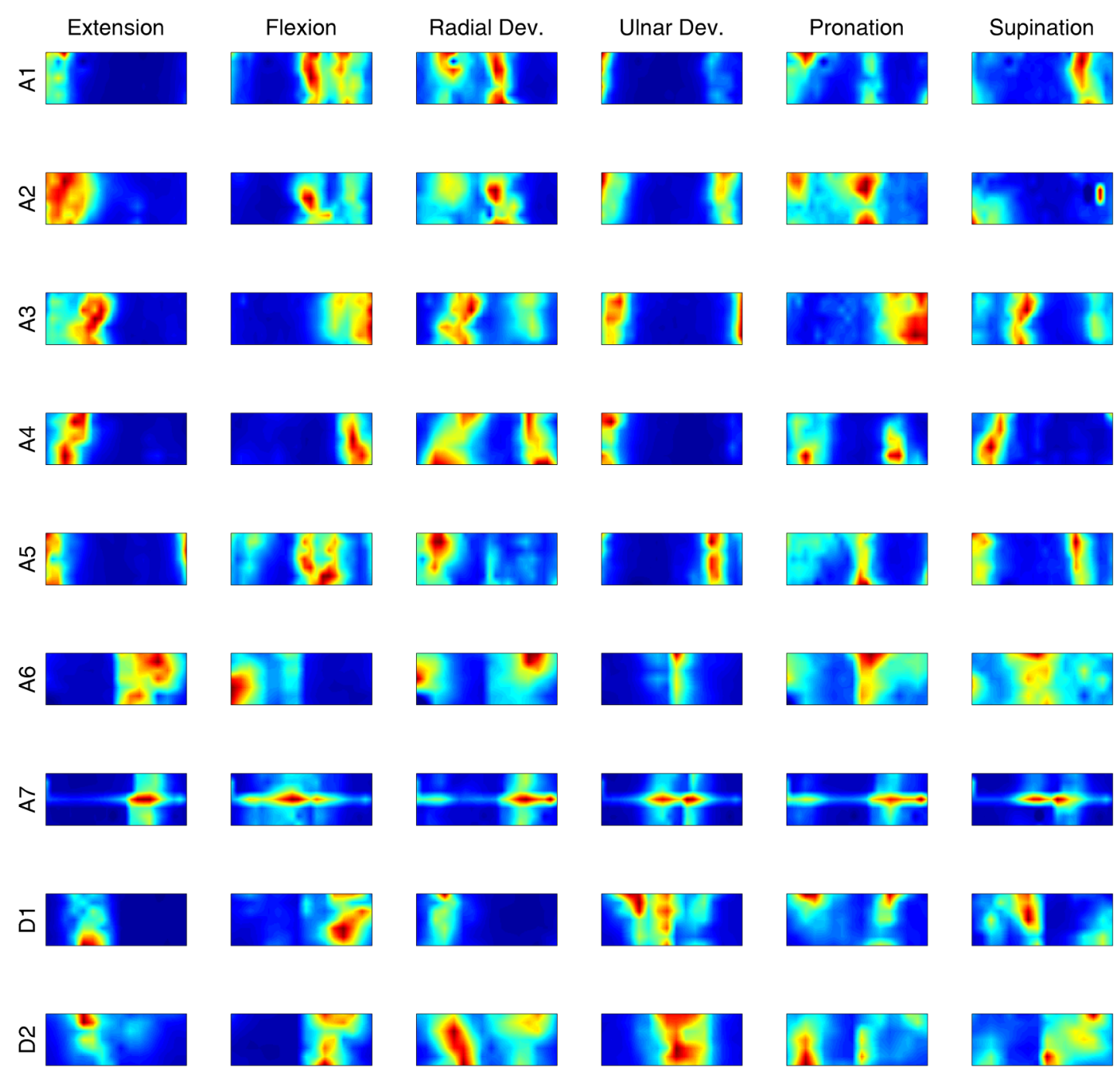

Figure 3.3.3: Surface representation of the motor tasks based on the normalized MUAP surface maps (C2016 IEEE). The sums of all normalized MUAP surface maps of the spike trains belonging to agonist are depicted for each motor task, with blue representing the smallest value within each map and red the largest. Able-bodied subjects are identified as A1-A7 and patients with limb deficiency as D1-D2. Note that since one motor unit can belong to agonist for multiple tasks, there is a considerable similarity between tasks for some subjects, even though discriminating between these is straightforward due to the large number of discriminative spike trains. A modified version of this figure also appeared in Kapelner, Negro, and Farina (2016).

3.4). The distributions of the angles at recruitment were skewed towards higher angles for all subjects (Figure 3.3.4). For three subjects the minimal normalized angle of recruitment was at least $20 \%$.

\subsubsection{Discussion}

This section showed that accurate surface EMG decomposition in voluntary contractions associated to prosthesis control with regression is possible. The decomposition extracted at least 15 spike trains per motor task on average in both able-bodied subjects and patients with limb-deficiency, despite the contractions being non-isometric. These results indicate 
A1

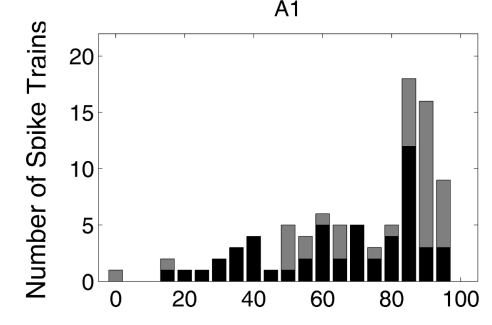

A4

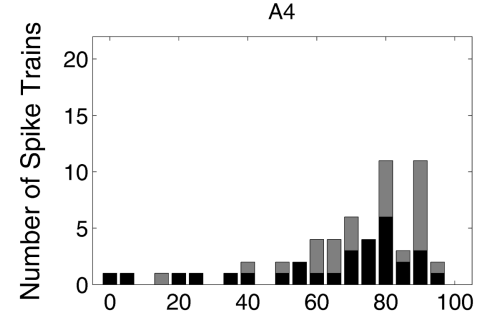

A7

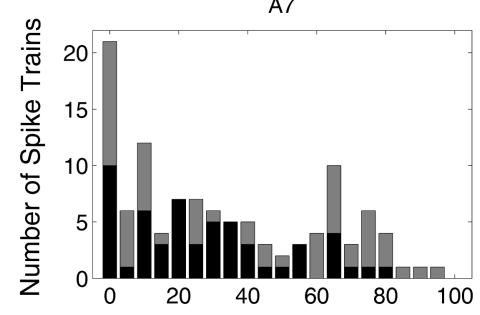

Normalized Angle at Recruitment [\%]
A2

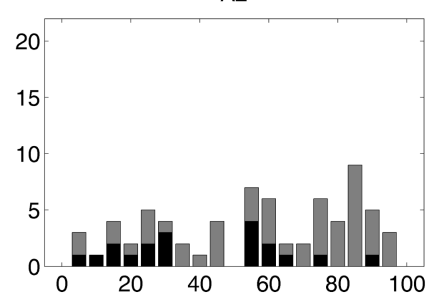

A5

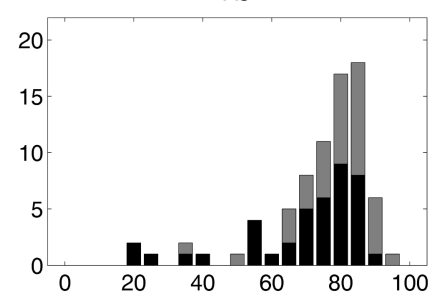

D1

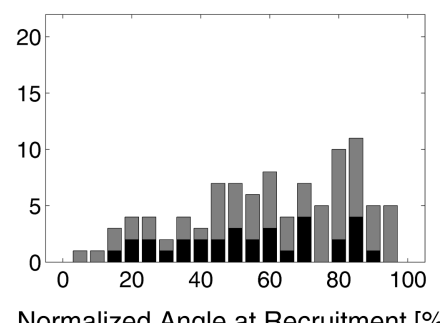

A3

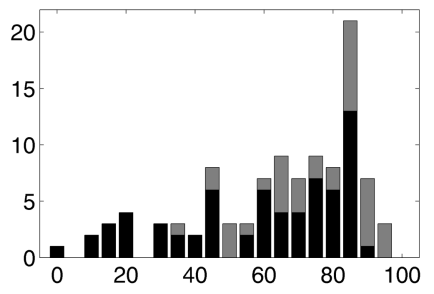

A6

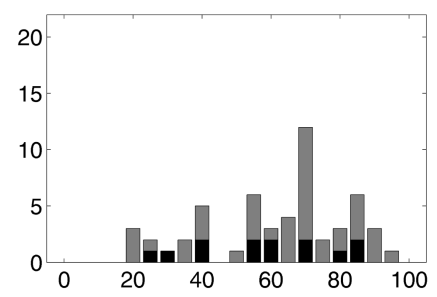

D2

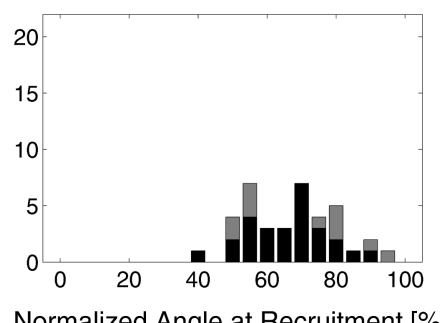

Figure 3.3.4: Histograms of the normalized angles at recruitment for each subject (О2016 IEEE). The height of the grey bars represent the total number of spike trains, and the height of the black bars represent the number of units among these that were detected consistently in all repetitions of a given motor task. A modified version of this figure also appeared in Kapelner, Negro, and Farina (2016).

that advanced prosthesis control by using neural information for regression is feasible.

The number of decomposed spike trains was similar for all tasks, but there was considerable variation in the number of agonist and consistently present units. (Tables 3.3 and 3.4). In most cases not all detected units were agonists, and there were fewer consistently present units than agonists. Nevertheless, in 6 out of 9 subjects consistently detected discriminative motor units were detected for at least 5 out of 6 tasks. These units are especially important for myoelectric control, since a control method could perfectly classify the active motor task based on their presence. This finding together with the high number of decomposed spike trains suggests that prosthesis control using automatically decomposed spike trains is feasible.

Another finding that shows that the discrimination of movements would be facilitated by neural information is that in subjects with limb-deficiency there were no motor units active during more than two tasks. One the one hand this might be a result of the muscles after amputation being artificially arranged, which leads to less similar MUAPs and facilitates prosthesis control (Figure 3.3.3). On the other hand, it is also possible that prosthesis users have learned how to activate individual muscles for optimal prosthesis control, whereas able-bodied subjects used their muscles synergistically. 
The findings in this section also confirmed the findings of Section 3.2, in that in spite of similarities in the surface representations of the motor tasks, it is possible to directly identify the active motor task using neural information, by detecting the presence of discriminative agonist units. Thus, neural information might offer more selectivity and potentially more accurate control compared to surface EMG features.

In general the distribution of normalized angles at recruitment was skewed towards higher angles (Figure 3.3.4). For subjects A5, A6 and D2, the smallest angle was greater than $20 \%$. Although the muscle force - wrist angle relationship is highly non-linear, because of the gradual increase in angle, motor units recruited at higher angles were likely to exhibit higher recruitment thresholds. Thus, the majority of detected motor units were presumably large, high threshold units, which are indeed simpler to detect because of their larger innervation numbers and therefore larger surface MUAPs (Farina et al. 2010; Henneman 1957).

Nevertheless, this finding shows that a prosthesis control methods based on neural information will have to cope with the fact that the number of motor units detected at small wrist angles might not be sufficient for extracting control information, since in three subjects there were no motor units at all detected below $20 \%$ of the maximal angle of movement.

Another limiting factor might be that less than half of the decomposed spike trains were present in all repetitions of a given motor task. This might be because of MUAP waveform changes, as described in Section 2.3. This effect is, however, not critical for prosthesis control, since as long as the motor task can be identified based on motor units, detecting the exact same motor neurons in all repetitions of a task is not strictly necessary.

The found SIR values were smaller compared to results previously shown for static contractions (Hamid R. Marateb et al. 2011). This is consistent with high angles at recruitment and few consistently present spike trains, both indicating that the extracted motor units only accounted for a fraction of the total EMG power. Thus, although decomposition with available methods is possible, specialized methods suited for strongly non-stationary conditions would be needed to mitigate these effects (De Luca et al. 2015; Sandercock et al. 1985).

\subsubsection{Summary}

This section showed for the first time that accurate identification of motor neuron activity from EMG decomposition during non-isometric voluntary contractions of the wrist is possible, in both able-bodied and limb-deficient subjects. The identification was accurate, discriminative for the tasks, and reliable across repetitions. These characteristics show that prosthesis control using such information is feasible, although some considerations will have to be made in order to mitigate the effects of incomplete decomposition. The advanced prosthesis control framework presented in the next section will take these considerations into account. 


\subsection{Study 4: Linear regression based on neural information}

The results of this study are submitted for publication in Kapelner, Jiang, and Farina (2016). Upon acceptance the copyright will be transferred to IOP. However, the authors are granted back a number of rights from IOP, including the inclusion of the article in a dissertation. Thus, permission was not required.

Author contributions: performed the experiments, analyzed the data, wrote the manuscript.

\subsubsection{Introduction}

The previous sections have shown that neural information outperforms EMG-based features in pattern recognition, and that the neural information provided by current decomposition methods is sufficient for regression. In this section the performance of linear regression using neural features will be compared to EMG-based features, as the first step towards a truly neural non-invasive prosthesis control.

As discussed previously, relating neural features to continuous joint kinematics could potentially increase regression performance, due to the inherent relationship between the motor neuron discharge rates and muscle force. As described in Secion 2.1, this relationship is generally nonlinear, also reflected by nonlinear estimators outperforming linear ones in offline regression (Jiang et al. 2014).

Nevertheless, for this first step in providing a proof of concept for neural information based prosthesis control, linear regression will be used for both EMG based and neural features (Hahne et al. 2012; Hahne et al. 2014). The development of the novel regression method was done taking the following observations of the previous sections into account.

Firstly, Section 3.3 found that the decomposition usually results in just a few spike trains at low joint angles. Secondly, a compensation mechanism for not consistently present spikes is also to be considered. Lastly, Section 3.2.4 highlighted that grouping of motor units is only beneficial if the involved units have functional similarities. These previous findings constituted the basic requirements for developing the control method described in the following.

To further investigate the effects underlying these previous observations, the experiments were performed in several speeds and movement ranges. The expected dependence of performance on speed was that since EMG decomposition complexity increases with the contraction force, neural information based control performance should decrease with increasing contraction intensity (Holobar et al. 2011). On the other hand previous research suggests that fine movements of forearm muscles corresponding to weak contractions should correlate more with neural information than with conventional global EMG features (Farina et al. 2010). This effect could however be influenced by an insufficient number of motor units decomposed at low wrist angles, as suggested by Section 3.3.3. 


\subsubsection{Methods}

\section{Data acquisition and experiment protocol}

Subjects A1-A6 and D1 from Section 3.3 participated in this study. The data acquisition and the experiment protocol were the same as described in Section 3.3.2 In that section the subjects were instructed to perform voluntary contractions of the wrist in three DoF in a triangular angle profile, reaching the maximal range of movement from rest position in $2.5 \mathrm{~s}$. In this experiment the protocol contained identical instructions, but with two additional timings: using $1 \mathrm{~s}$ and $5 \mathrm{~s}$ ramps. Additionally, subjects A4-A7 were instructed to perform the same protocol using only $20 \%$ of their range of movement. All participants gave informed consent prior to the experiment, as approved by the local ethics committee.

\section{EMG Feature Extraction}

The raw EMG was digitally band pass filtered with cut-off frequencies 20 and $500 \mathrm{~Hz}$. Then the signal was windowed in 100-ms intervals, with $10 \mathrm{~ms}$ overlap, and the same TD features were extracted as described in Section 3.2.2 (Hudgins, Parker, and Scott 1993). The necessary threshold parameters were selected manually for each subject. Afterwards data dimensionality was reduced using PCA, so that the resulting signal retained $98 \%$ of the original variance. This feature set will be referred to as TD.

\section{EMG decomposition and spike processing}

The recorded EMG signal was decomposed offline using the CKC algorithm for each DoF separately, in the same manner as in Section 3.3. (Holobar and Zazula 2007). After decomposition motor units present in more than one DoF were identified by using the cross-correlation of spike triggered averaged MUAPs.

Because the spike triggered averages contained more noise due to shorter contractions compared to the previous section, the cross correlation differed from the one described in Section 3.3.2, in that MUAPs in channels containing artefacts or noise were not included.

All channels were included in the cross-correlation calculation, for which the ratio of the maximal absolute value within and outside the MUAP duration (as defined in Section 2.3 ) was below $80 \%$ of the maximal ratio of that MUAP. This means that if the signal amplitude outside the time support was comparable to the one within, the channel was not included in the correlation calculation (Figure 3.4.1).

To ensure the robustness of the neural information based feature set, the number of firings was calculated for the same observation windows as for TD. This feature set will be referred to as Decomposed Spike Counts (DSC) in the rest of the thesis. Similarly to TD, the dimensionality of DSC was reduced using PCA retaining $98 \%$ of the signal variance.

To compensate for the missing information suggested by the previous section, two approaches were compared.

In the first compensation approach the residual EMG signal was added to DSC. The calculation of the residual was carried out by using the numerator of (3.1), in which the 
A

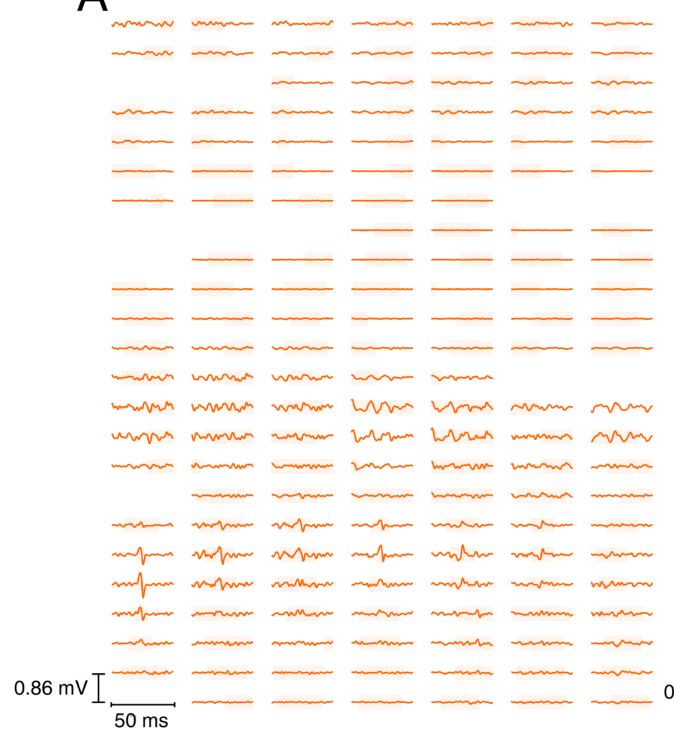

B

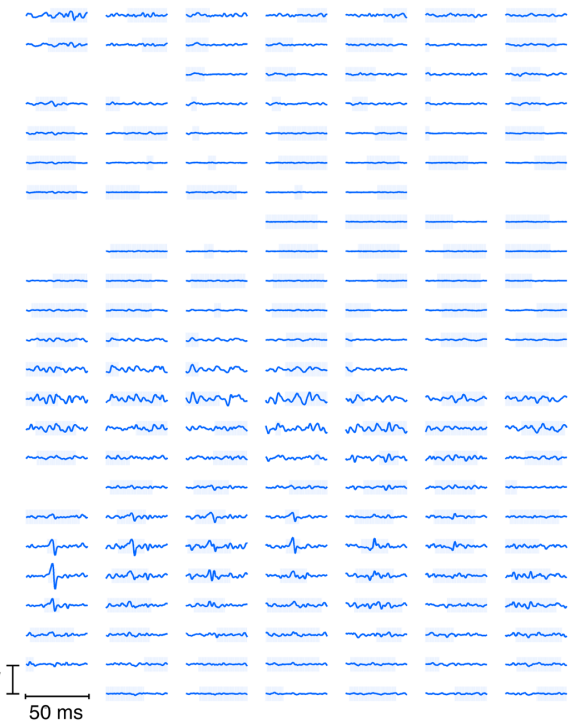

C

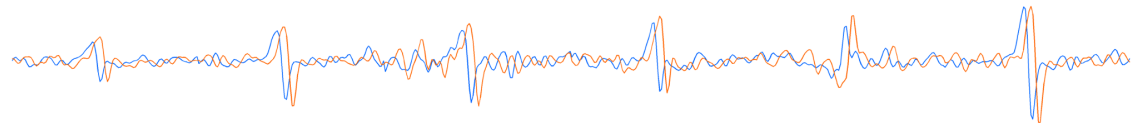

Figure 3.4.1: A representative example of the comparison method based on $M U A P$ shapes. A and B show the MUAP shapes of two motor units decomposed at different DoFs, considering all three electrode matrices as one large matrix. One of the shapes is shown in blue, the other in orange. The two spike triggered averages are similar in terms of meaningful action potentials, as seen in the lower left corner of both images. However, due to large uncorrelated noise components in the middle two rows of the electrode matrix, the maximum of the cross-correlation function between the two waveforms is 0.6188. Using the method described above, the cross-correlation only considers channels actually containing MUAP shapes, depicted in C. The more accurate cross correlation based on these signals was 0.9077. This figure also appeared in Kapelner, Jiang, and Farina (2016).

EMG reconstructed by the summation of MUAP shapes was subtracted from the measured EMG. TD features of the residual signal were calculated and signal dimensionality was reduced using PCA to retain $95 \%$ variance. The resulting multichannel signal together with DSC constituted the DSC $\mathrm{RES}_{\text {f }}$ feature set. The inclusion of the raw EMG effectively meant that the control method will only use neural information when it is available, in other cases the control will revert to traditional regression.

In a second approach to mitigate the effect of missing information, a model-based method based on physiological considerations was used in addition to $\mathrm{DSC}_{\mathrm{RES}}$ to promote robustness. Thereby I have devised grouping scheme for spike trains inspired by motor unit physiology, as introduced in Section 2.1., and also considering the observations of Section 3.2. In this sense this method is a neural information based alternative to PCA dimensionality reduction.

\section{Model-based dimensionality reduction}

Motor neurons residing in the same muscle - or within synergistic muscle pairs - share a large part of their synaptic input (De Luca and Erim 1994, 2002; Farina, Negro, and 
Dideriksen 2014). Thus, as described in Section 2.1, a single motor unit spike train carries information about the activity of the whole muscle, which is connected to the activity of all motor units that receive the same input. Modeling this relationship would allow to neglect the effect of missing spikes or false positives of one spike train, as long as others are decomposed accurately.

This redundancy can be modeled by groups of motor units estimating the same target force. Each unit $j$ in the group $i$ has an individual relationship $g_{i j}($.$) with an individual \alpha_{i j}$ estimate of muscle force, which is used to calculate the common activation level $a_{i}$, of the whole group (Figure 3.4.2). The model $g_{i j}($.$) could theoretically include all physiological$ processes mentioned in Section 2.1. In this section a linear model with intercept will be used for simplicity.

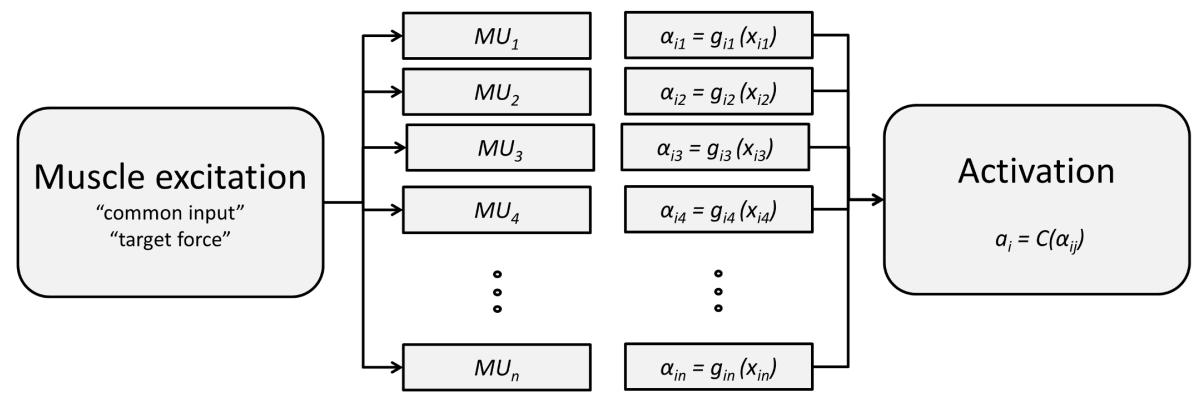

Figure 3.4.2: The proposed dimensionality reduction model (right) and the physiological model that it is inspired by (left). Each motor unit receives a common input signal from the CNS, which corresponds to each decomposed spike train individually estimating a common activation level. This figure also appeared in Kapelner, Jiang, and Farina (2016).

The common activation of the $i$-th group $K_{i}$ was obtained by combining the individual $\alpha_{i j}$ estimates with a robust method $C($.$) :$

$$
a_{i}=C\left(\alpha_{i j}\right)=\underset{j \in J_{i}}{\operatorname{median}} \alpha_{i j}
$$

where each motor unit $j$ of the group $J_{i}$ had at least one spike during the last 200 $\mathrm{ms}$, to exclude inactive units. To promote the smoothness of the estimate the previously estimated force was also included in the median. These computationally trivial steps added another layer of robustness to the framework.

The resulting $a_{i}$ activations were then used together with the residual EMG to linearly estimate joint kinematics. Thus, the model presented in Figure 3.4.2 is a signal processing 
step before regression, converting DSC into activations and thereby reducing its dimensionality. The output of this model will be referred to as the $\mathrm{DSC}_{\mathrm{MOD}}$ feature set.

It is worth noting that this method is suitable to use with any neural information extraction approach besides surface EMG decomposition, such as online EMG decomposition algorithms of intramuscular EMG (Negro et al. 2016), as well as other methods such as peripheral nerve electrodes (Boretius et al. 2010; Lago et al. 2005; Micera et al. 2008).

\section{Linear regression}

Linear regression was implemented using the built-in linear model in MATLAB. At the output of the linear regression the median of three consecutive samples was taken to improve performance.

Three-fold cross validation was used to assess the performance, which was quantified by the $\mathrm{R}^{2}$ goodness of fit measure (d'Avella et al. 2006). In each fold, one DoF triangle for each direction of each DoF was designated as testing data, and the remaining two triangles for training. The cross-validation was repeated 10 times.

The model on which dimensionality reduction scheme of $\mathrm{DSC}_{\mathrm{MOD}}$ was based had to be trained as well. Each $a_{i}$ was defined as one direction of one DoF, e. g. $a_{1}$ and $a_{2}$ were set equal to the positive and negative directions of $\mathrm{DoF}_{1} ; a_{3}$ and $a_{4}$ to those of $\mathrm{DoF}_{2}$ etc. (Figure 3.4.3). Then, motor units were grouped together based on the activations: the motor unit $j$ was assigned to the activation $a_{i}$ that had the highest correlation with its spike train. Motor units that had a positive correlation with both directions of the same DoF were disregarded.

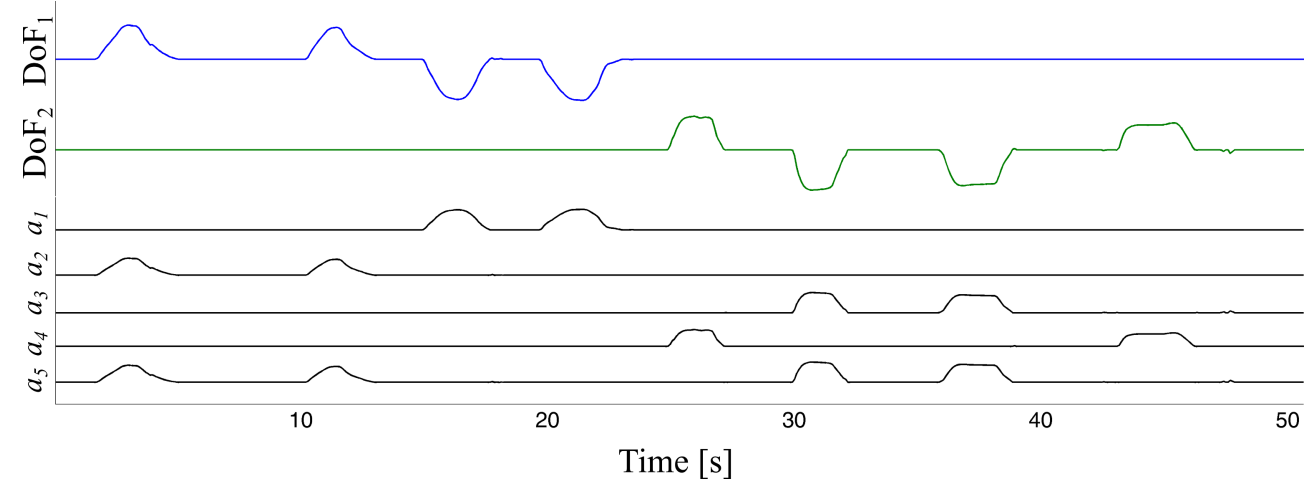

Figure 3.4.3: Representative examples of the training of the activations estimated by groups of motor units. Here $a_{1}$ and $a_{2}$ estimate the two directions of DoF $F_{1}$, and $a_{3}$ and $a_{4}$ those of $D o F_{2}$. $a_{5}$ represents a common activation of the positive directions of $D o F_{1}$ and the negative direction of $D o F_{2}$. In this example the randomized order of the DoF triangles and $\mathrm{DoF}_{3}$ are eliminated for clarity. This figure also appeared in Kapelner, Jiang, and Farina (2016).

The result of this process was a grouping between all motor units, with units of the same group active during the same direction of the same $\operatorname{DoF}(\mathrm{s})$. This ensured the functional similarity of the grouping, which is an important criterion for control based on Section 3.2.4. The model parameters of each $g_{i j}()$ relating DSC to the activation estimates were trained using linear optimization. 


\section{Statistical analysis}

Mean \pm standard deviation of the $\mathrm{R}^{2}$ was used as descriptive statistics and ANOVA was conducted to assess differences between features $(\alpha=0.05)$. First a full ANOVA model was used with the fixed-level factors "Feature" and "Ramp Duration", and the random factor "Subject" nested within the fixed factor "Group" with levels "Able Bodied" and "Limb Deficiency". Differences in features only were analyzed using one-way ANOVA with repeated measures with the constant factor "Feature", for each subject and ramp duration separately. Differences in movement speed were analyzed using one-way ANOVA for each subject and feature with the constant factor "Ramp Duration". For reduced movement range the above calculations were repeated, and differences in movement range were analyzed with two-sample Student's t-tests for each condition separately. Post-hoc tests were conducted using Bonferroni's correction.

\subsubsection{Results}

For both movement ranges, statistically significant differences were observed between features $(p=0.028)$ and groups $(p=0.048)$, and significant interactions between the factors Feature-Subject $(p=0.02)$ and Speed-Subject $(p<0.001)$, as well a three-way interaction between the factors Subject-Feature-Speed $(p<0.001)$. Thus, the control performance for each subject and ramp duration had to be analyzed individually, as described in 3.4.2 Methods.

\section{EMG decomposition}

The decomposition of the EMG signals was successful, even though the contractions were more than twice as fast as those of Section 3.3. In general the number of decomposed motor units increased with ramp duration, and decreased with movement range (Table 3.5 .

Table 3.5: The mean and standard deviation of decomposed spike trains, sorted by the DoF they were active in. The last column indicates the mean of all decomposed units per subject. Note that the number of subjects for the full movement range is seven, whereas for the $20 \%$ range it is four. This table also appeared in Kapelner, Jiang, and Farina (2016).

\begin{tabular}{|c|c|c|c|c|c|c|c|c|}
\hline \multicolumn{2}{|c|}{ Ramp duration } & $\operatorname{DoF}_{1}$ & $\mathrm{DoF}_{2}$ & $\mathrm{DoF}_{3}$ & $\begin{array}{c}\mathrm{DoF}_{1}+ \\
\mathrm{DoF}_{2}\end{array}$ & $\begin{array}{c}\operatorname{DoF}_{1}+ \\
\operatorname{DoF}_{3}\end{array}$ & $\begin{array}{c}\mathrm{DoF}_{2}+ \\
\mathrm{DoF}_{3}\end{array}$ & Total \\
\hline \multirow{3}{*}{$\begin{array}{c}\text { Full } \\
\text { movement } \\
\text { range }\end{array}$} & $1 \mathrm{~s}$ & 0.4 & $3.1 \pm 9.2$ & $26.0 \pm 7.5$ & $1.4 \pm 1.2$ & $0.7 \pm 0.9$ & $2.0 \pm 1.7$ & $101.8=$ \\
\hline & $2.5 \mathrm{~s} \mathrm{ramp}$ & $24.3 \pm 10.7$ & $22.3 \pm 6.8$ & $23.7 \pm 6.9$ & $2.4 \pm 1.4$ & $1.3 \pm 1.3$ & $2.3 \pm 1.3$ & $76.3 \pm 24.1$ \\
\hline & $5 \mathrm{~s} \mathrm{r}$ & $15.0 \pm 2.8$ & $16.3 \pm 6.3$ & $16.0 \pm 4.6$ & $3.0 \pm 3.2$ & $1.3 \pm 0.9$ & $3.0 \pm 1.4$ & $54.6 \pm 11.1$ \\
\hline \multirow{3}{*}{$\begin{array}{c}20 \% \\
\text { movement } \\
\text { range }\end{array}$} & $1 \mathrm{~s} \mathrm{r}$ & $13.5 \pm 7.8$ & $15.3 \pm 6.9$ & $6.0 \pm 3.2$ & $3.0 \pm 2.2$ & $1.8 \pm 1.0$ & $2.8 \pm 2.2$ & $42.3 \pm 20.7$ \\
\hline & $2.5 \mathrm{~s}$ & $10.8 \pm 3.4$ & $14.5 \pm 4.4$ & $8.0 \pm 0.8$ & $3.0 \pm 3.2$ & $2.5 \pm 1.3$ & $2.8 \pm 1.0$ & $41.5 \pm 7.7$ \\
\hline & $5 \mathrm{~s}$ ramp & $11.3 \pm 3.6$ & $11.0 \pm 5.7$ & $4.8 \pm 2.1$ & $3.8 \pm 3.6$ & $1.3 \pm 1.0$ & $2.0 \pm 2.8$ & $34.0 \pm 9.5$ \\
\hline
\end{tabular}

\section{Linear regression performance}

In the full movement range TD significantly outperformed DSC and both $\mathrm{DSC}_{\mathrm{RES}}$ and $\mathrm{DSC}_{\mathrm{MOD}}$ significantly outperformed TD in almost all cases. There was no pattern to 
the exceptions (Figure B.1 in Appendix B). In the reduced movement range all neural information based features outperformed TD in almost all cases. In general $\mathrm{DSC}_{\mathrm{MOD}}$ proved to be the best performing feature set (Figure B.2 in Appendix B).

The dependence of feature performance on ramp durations showed an inconsistent, subject and feature specific pattern for both movement ranges. For some subjects performance increased with increasing movement speed, for others the trend was the opposite, or there was no trend to observe. This was true for all feature sets and movement ranges (Figures B.3 and B.4 in Appendix B). The least performance difference between different ramp durations was observed for $\mathrm{DSC}_{\mathrm{MOD}}$.

The performance of all feature sets was significantly higher in the full range of movement, except for DSC in some cases (Figure B.5 in Appendix B). A detailed description of individual comparisons between features, ramp durations and movement ranges can be found in Appendix B, an overall comparison of performance across all conditions is shown in Figure 3.4.4.

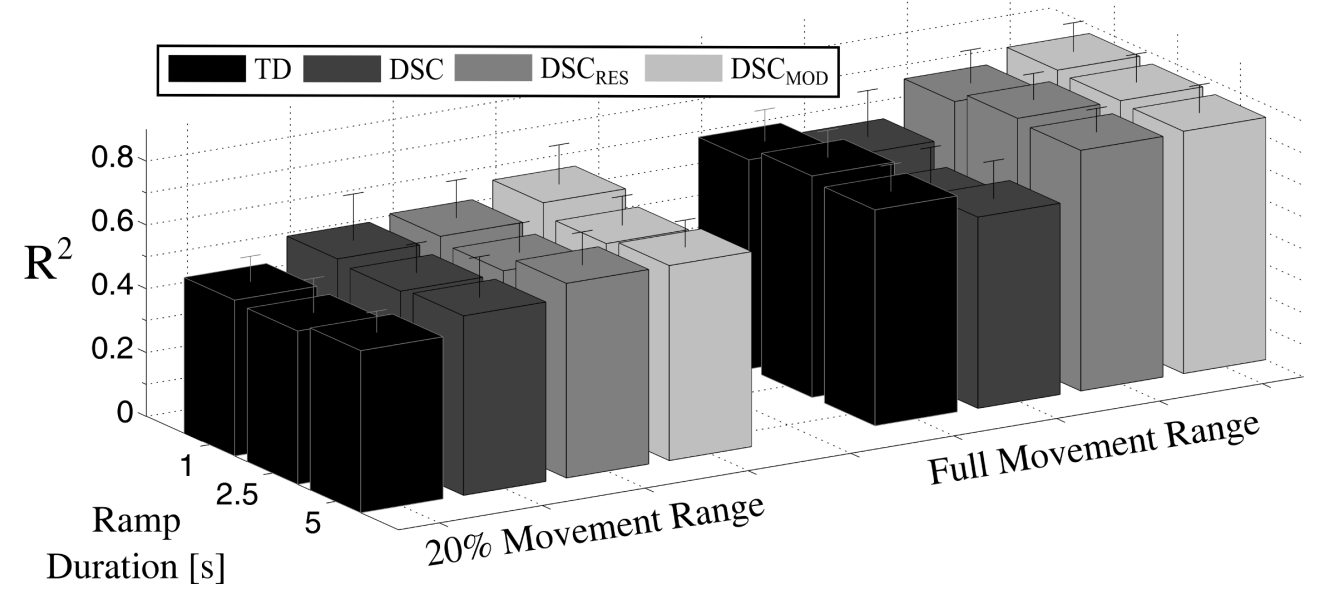

Figure 3.4.4: An overview of the results combining the data of all subjects. The relationship between the factors is detailed in Appendix B. This figure also appeared in Kapelner, Jiang, and Farina (2016).

\subsubsection{Discussion}

The primary finding of this section is that the developed novel control method based on neural information outperformed traditional EMG-based linear regression. However, to achieve this result the effect of missing information had to be reduced, either by including the residual EMG in the feature set or using a robust control method in addition. Regression performance was influenced by movement speed and movement range in a subject-specific manner.

\section{EMG decomposition}

The findings of this section reinforced the conclusion of Section 3.3, in that while it is possible to decompose EMG signals recorded during voluntary dynamic contractions, the 
presence of missing information requires a robust control method. As for the dependence on contraction speed, more spike trains were decomposed at shorter ramp durations than at longer.

This suggests that the contractions at higher speeds might have been stronger, and thus more motor units were recruited within the pick-up area of the electrodes. However, $\mathrm{DSC}_{\mathrm{MOD}}$ outperformed all other features in this condition, suggesting that a robust compensation method was needed to mitigate the effect of missing information.

Another interesting finding was that although the number of decomposed spikes was lower for the $20 \%$ movement range, there was a considerable amount of decomposed spikes. Since the in the previous section there were just a few spikes present in this range of movement, this finding suggests that decomposing the EMG at lower force levels is only problematic if higher force levels are also present in the signal. Future EMG decomposition methods specific to this application will have to consider this effect.

\section{Linear regression performance}

For the reduced movement range, corresponding to low force contractions, neural information showed better control performance, even without mitigating the effect of missing information. This was expected because on the one hand the EMG noise at low contraction levels is comparable with the activation, decreasing regression performance. On the other hand, neural spike trains are more correlated to muscle force oscillations compared to EMG amplitude (Farina et al. 2010).

However, for the full movement range regression performance of neural information was inferior to traditional features, which was also expected based on the results of Section 3.3. Besides missing information another reason for this low kinematics estimation accuracy may have been the non-linear relationship between discharge rate and wrist kinematics. Nevertheless, DSC $_{\mathrm{RES}}$ and $\mathrm{DSC}_{\mathrm{MOD}}$ both outperformed TD, indicating that spike trains carry additional information not available in EMG-based features, even for high channel numbers.

The inferior performance in the reduced movement range for all features except DSC can be explained by the task difficulty and noise. Firstly, precise movements are harder to control in general, and absolute error results in a higher relative error at the smaller ranges. Secondly, the low EMG signal to noise ratio at low contraction levels might have impeded the control performance of TD. The better performance of DSC for some subjects could be explained by subject specific distributions of motor unit recruitment thresholds, as discussed below.

Overall the best performing feature set $\mathrm{DSC}_{\mathrm{MOD}}$ (Figure 3.4.4), although a significant advantage over $\mathrm{DSC}_{\mathrm{RES}}$ was only observed at the fastest speed. This is likely because the amount of missing information was higher for faster contractions, and the model-based robust control approach was capable to mitigate this effect the most. 


\section{Dependence on Movement Speed}

The statistically significant differences between ramp durations were subject and feature specific, and inconsistent across movement ranges. Decreasing neural information based regression performance with increasing movement speed was only confirmed in two out of six subjects. This shows that, contrary to previous expectations, regression performance depends on a number of factors other than decomposition complexity.

The most important confounding factor may be that the distributions of recruitment thresholds of motor units picked up by the decomposition algorithm are not uniform for each subject. For instance, it is possible that the sample of one subject contained a relatively large number of medium threshold units, activated in both $2.5 \mathrm{~s}$ and $5 \mathrm{~s}$ ramp durations, but suppressed by larger units and noise at $1 \mathrm{~s}$ ramp duration. In this subject the regression performance of $1 \mathrm{~s}$ ramp duration would be smaller for the reduced movement range.

On the other hand, some of the medium threshold units might have had a higher recruitment threshold than the forces exerted at reduced movement range for the same subject. Thus, decreasing the movement speed will decrease regression performance in that range of movements. In our subject pool we observed such a relationship between factors for subject A6. For the other subjects, corresponding scenarios can also be constructed.

This explanation highlights the possible interactions between the factors of the ANOVA analysis. Unfortunately, based on this experiment it is not possible to confirm this hypothesis, since a measurement of individual muscle forces with the same electrode configuration during the same intended movements was not feasible.

Besides this possible explanation, other confounding factors include subject anatomy, the relative movement of tissue layers in dynamic contractions, the composition of the tissue filter and changes in recruitment thresholds due to contraction speed changes (Masakado et al. 1995).

\subsubsection{Summary}

This section introduced the first proof of concept for a neural information based prosthesis control method using surface EMG decomposition. This method outperformed EMG-based linear regression in both able bodied subjects and patients with limb deficiency. For precise wrist deviations within $20 \%$ of the full movement range superior performance could be achieved using only neural information, whereas for the full range of movement missing information had to be compensated. Neural features with the novel dimensionality reduction method proposed in this section showed better overall performance than all other investigated features.

Since the relationship between the investigated features in and wrist kinematics is nonlinear, it is possible that the results of this section only reflect the suitability of the different features for linear estimation, as opposed to overall predictive value. In order to fully characterize the relationship between EMG-based and neural information based kinematics estimation, it is necessary to compare their performance in a nonlinear setting. 
This would also be an opportunity to fully exploit neural information by modeling the physiology of the wrist hand system during movement. This investigation is included in the next section. 


\subsection{Study 5: Neuromusculoskeletal modeling based on neural informa- tion for prosthesis control}

The results of this study are published in Kapelner, Sartori, and Farina (2016). Upon acceptance the copyright will be transferred to IEEE. In accordance with the requirements of the copyright holder, figures reused in this section are indicated by the copyright line (C) 2016 IEEE, and the approval of the senior author, Prof. Dario Farina, was obtained to reuse a substantial portion of the original paper.

Author contributions: performed the experiments, analyzed the data, wrote the manuscript.

\subsubsection{Introduction}

The previous sections explored several aspects of prosthesis control using neural information obtained from the surface EMG. This section will use the experience of those investigations and previous research on the physiology of the wrist-hand system to implement a non-linear kinematics estimation method for prosthesis control using EMG decomposition. This method will be compared to state of the art regression using machine learning in terms of offline regression accuracy.

Biomechanical models of the wrist use accurate muscle, tendon and joint models to estimate muscle force, joint dynamics, and joint kinematics based on muscle excitations and physiological constraints (Buchanan et al. 2004; Lemay and Crago 1996; Majors and Wayne 2011). In recently developed neuromusculoskeletal (NMS) models the muscle excitations are estimated using EMG signals acquired from the skin over specific muscles (Lloyd et al. 2008; Sartori, Farina, and Lloyd 2014).

As discussed in Section 2.1, the central nervous system uses neural control signals to drive our muscles during movement. Thus, as an alternative to EMG, muscle excitations could be estimated from the neural drive to increase the accuracy of NMS modeling (Sartori and Farina 2016). As a final step of this series of investigations, in this section a novel NMS model will be proposed that uses neural information to estimate wrist kinematics.

The proposed model uses neural discharges acquired from surface EMG to estimate muscle excitations, thereby modeling human movement on a neural level as well as on a muscle level. The estimated excitations serve as an input to a biomechanical model that estimates the kinematics of the wrist during movement (Figure 3.5.1). In this section the proposed model will be described in detail and its performance will be compared to state of the art nonlinear regression using an ANN.

\subsubsection{Methods}

\section{Data acquisition and experiment protocol}

Subjects A1-A6 and patients D1 and D2 from Section 3.3 participated in this study. The experiment setup and the data acquisition method was the same as described in Section 3.4.2. The experiment protocol was also the same as described in Section 3.4.2, restricted to the shortest ramp duration (1s) of the triangular wrist angle profiles (Figure 3.5.2). 


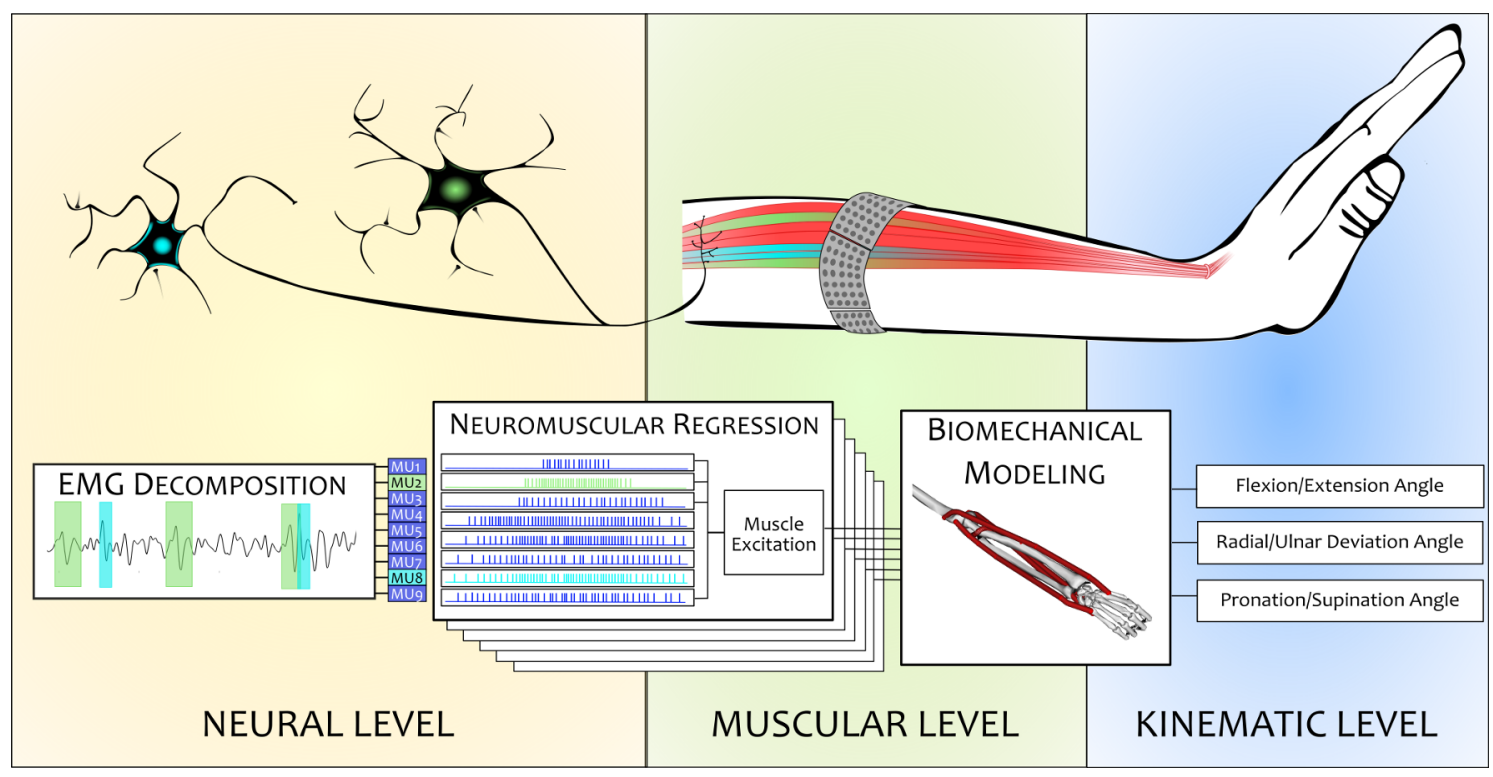

Figure 3.5.1: An illustration of the NMS modeling framework proposed in this section. Neural information obtained from EMG decomposition is used in estimate muscle excitations using neuromuscular regression. The information is thereby translated to the muscular level, and used by a biomechanical model to estimate the kinematics of the wrist.

The recorded EMG signals were decomposed using (Negro et al. 2016), and the TD and DSC feature sets were calculated as described in Section 3.4.2, without dimensionality reduction for DSC - this is the same procedure as was done for the feature set $\mathrm{DSC}_{\mathrm{MOD}}$ in Section 3.4.2.

To evaluate whether there is an increase in regression performance due to including both neural information and EMG features but without using a biomechanical model, a third feature set containing both TD and DSC was introduced, for which the same dimensionality reduction was applied as for TD.

\section{Artificial Neural Network regression}

The nonlinear regression using EMG-based features was implemented with the neural network toolbox of MATLAB, using one separate network for each DoF and three neurons in one hidden layer per network, as suggested by (Hahne et al. 2014; Nielsen et al. 2011). The outputs of the networks were filtered using a three sample long median filter and a 2nd order low pass Butterworth filter with a cut-off frequency of $2 \mathrm{~Hz}$.

The training was done using the Levenberg-Marquardt back-propagation algorithm. For each fold 50 different ANNs were trained using two repetitions of each DoF, and only the network with the highest performance was used for further comparisons, as suggested by Jiang et al. (2012a). ANN regression was done using the TD and TD+DSC feature sets.

\section{Neuromusculoskeletal regression model}

The proposed NMS model consisted of two submodels (Figure 3.5.3). Firstly, a set of neuromuscular regression models were used to estimate muscle excitations based on the 


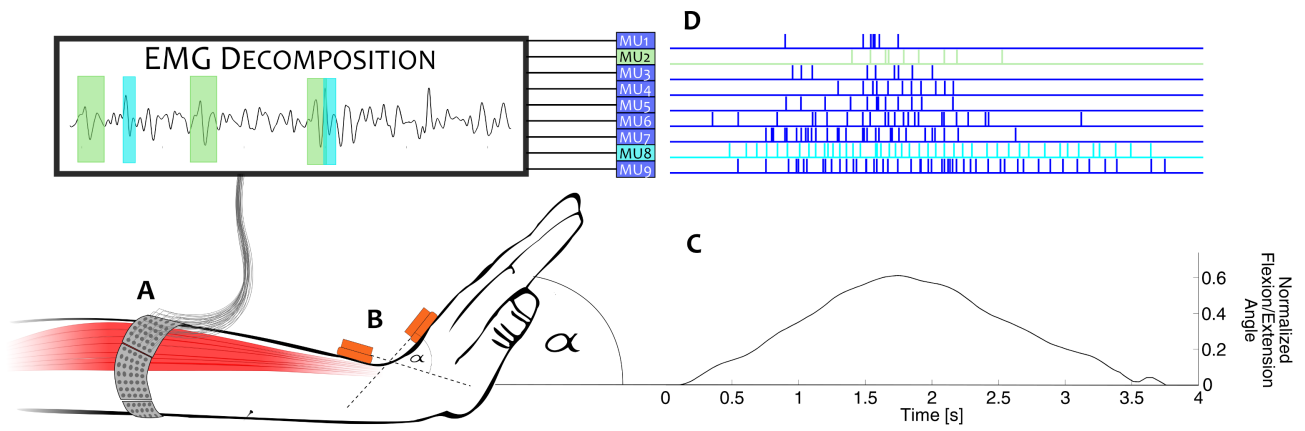

Figure 3.5.2: Illustration of the experiment protocol and data acquisition (@2016 IEEE). The subjects were equipped with HD surface EMG electrodes (A) and motion capture equipment $(B)$. The subjects performed voluntary movements of the wrist with a triangular joint angle profile guided by visual feedback $(C)$. Finally, the recorded EMG signal was decomposed into its constituent MUAP trains (C), and used for kinematics estimation. This figure also appeared in Kapelner, Sartori, and Farina (2016).

decomposed spike trains. The models were the same as the ones used for linear regression in Section 3.4.2. Then, a biomechanical model was used to estimate wrist kinematics from the estimated muscle excitations. In the following the biomechanical submodel will be described in detail, as well as the training and estimation phases of the whole system.

\section{(A)}

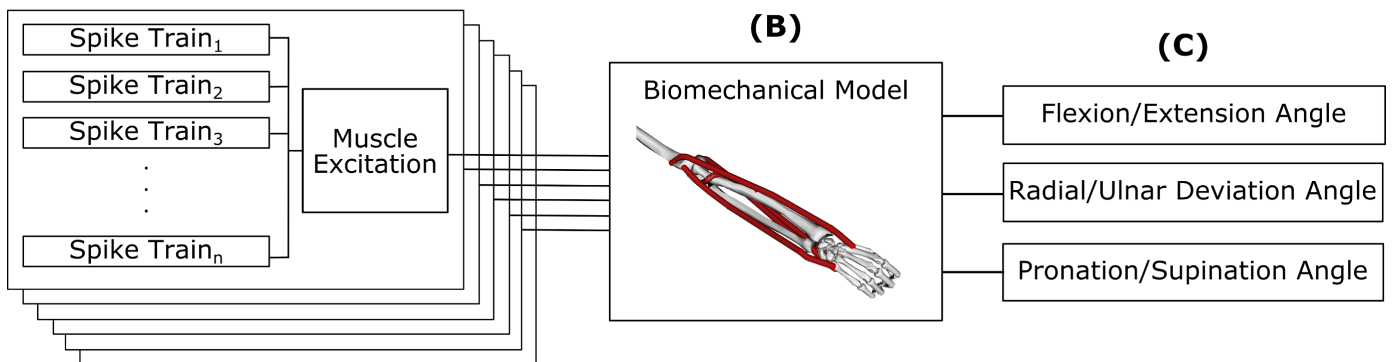

Figure 3.5.3: Block diagram of the proposed NMS model (@2016 IEEE). First a neuromuscular regression is used to estimate muscle excitations from neural discharges (left, first submodel). Then a biomechanical model (middle, second submodel) translates the estimated muscle excitations to kinematics (right). This figure also appeared in Kapelner, Sartori, and Farina (2016).

The biomechanical submodel was implemented using a generic model of the arm available in OpenSim (Delp et al. 2007; Saul et al. 2015). For simplicity the model was reduced to the wrist joint and the following muscles acting on it: musculus flexor carpi ulinaris (FCU), musculus flexor carpi radialis (FCR), musculus extensor carpi ulinaris (ECU), musculus extensor carpi radialis longus (ECRL), musculus pronator teres (PT), musculus suplinator (SUP). The maximal isometric muscle forces of the models were modified to have zero muscle excitations at rest, to remove the offset (due to passive forces) from the muscle excitation functions. The following three DoFs were estimated: flexion/extension, ulnar/radial deviation, and pronation/supination. 


\section{Model training}

The parameters of the functions in the neuromuscular regression models were trained using the same method as described in Section 3.4.2 (Figures 3.4.2 and 3.4.3), with one modification. Instead of activation functions derived from the output kinematics, the grouping of motor units was based on estimated muscle excitations, which were calculated using inverse kinematics from the biomechanical model. Assigning the decomposed motor units to muscles allowed for direct estimation of muscle excitation from the neural discharges, using the same method as in Section 3.4.

After scaling the recorded kinematics to the boundaries of the model, the training dataset was used to calculate muscle excitations by computed muscle control (CMC) (Thelen, Anderson, and Delp 2003). During the constrained optimization process of CMC non-agonist muscle excitations were restricted to zero at increasing absolute DoF angles to promote sparsity.

The decomposed spike trains were then matched to the computed muscle excitations in two steps. First a spike train was assigned to all muscles with an excitation $10 \%$ greater than the baseline at the time of at least $80 \%$ of the spike train's discharges. Finally the parameters of the robust linear estimator were trained to estimate the computed muscle excitation based on the DSC of the motor unit.

\section{Kinematics estimation}

Muscle excitations were estimated from DSC using the neuromuscular regression models and (3.3) (Figure 3.4.2). Then the estimated excitations were used to simulate wrist kinematics by forward kinematics estimation (Delp et al. 2007).

If there were no spike trains active in a given window, the TD feature set was used to estimate muscle excitations using one dedicated ANN for each muscle. The parameters and training method of these networks were the same as described for TD previously.

As shown by recent research, proportional control methods can be less accurate than pattern recognition in isolated single DoF movements (Smith, Kuiken, and Hargrove 2014). The detailed biomechanical information of the NMS model allowed for the limitation of unintended activations in other DoFs.

The NMS model estimates the excitation of all muscles, which are translated to DoFs through joint moments generated by muscle forces. For the purpose of prosthesis control this relationship was simplified to agonist-antagonist relations between muscles and DoFs. By only estimating kinematics in DoFs that were actuated by all its agonists, joint angles of inactivated DoFs could be set to zero, instead of letting them be estimated by the model.

For example, if both FCU and FCR were active, it was interpreted as a flexion and only flexion/extension was estimated (the others were set to zero). However, if in the above example any of the muscles were active alone, there was no information on whether flexion/extension or deviation should be active, and therefore in this case the method would estimate both. 


\section{Statistical analysis}

Three fold cross-validation was used to assess the regression performance of all features, using the same method as described in Section 3.4.

For statistical analysis I used nested ANOVA with the fixed factor "Feature" and the random factor "Subject", which was nested within the fixed factor "Group" (with levels "Able Bodied" and "Limb Deficiency"). Subsequent one-way repeated measures ANOVAs were then carried out for each subject individually, with the fixed factor "Feature". Posthoc tests were done using the Bonferroni correction with an alpha level of 0.05 .

\subsubsection{Results}

The decomposition yielded on average $19 \pm 7$ spike trains per DoF, corresponding to $63 \pm 16$ spike trains per subject (3.6). The results of the assignment of these spike trains to muscles by the NMS model, as described in the previous section, are detailed in Table 3.7.

Table 3.6: The number of decomposed spike trains per DoF for each subject (이이 IEEE). DoF $F_{1}$ was flexion extension, $D o F_{2}$ was radial/ulnar deviation, and $D o F_{3}$ was Pronation/Supination. Columns with more than one index contain spike trains detected in more than one DoF, e.g. DoF 123 contains spike trains active during all DoFs. This table also appeared in Kapelner, Sartori, and Farina (2016).

\begin{tabular}{c||c|c|c|c|c|c|c|c}
\hline & $\mathrm{DoF}_{1}$ & $\mathrm{DoF}_{2}$ & $\mathrm{DoF}_{3}$ & $\mathrm{DoF}_{12}$ & $\mathrm{DoF}_{13}$ & $\mathrm{DoF}_{23}$ & $\mathrm{DoF}_{123}$ & Total \\
\hline \hline $\mathrm{A} 1$ & 16 & 16 & 30 & 0 & 0 & 3 & 0 & 65 \\
\hline $\mathrm{A} 2$ & 7 & 17 & 9 & 3 & 0 & 4 & 1 & 40 \\
\hline $\mathrm{A} 3$ & 23 & 21 & 11 & 2 & 2 & 1 & 0 & 60 \\
\hline $\mathrm{A} 4$ & 18 & 30 & 22 & 4 & 0 & 0 & 0 & 74 \\
\hline $\mathrm{A} 5$ & 4 & 12 & 18 & 3 & 1 & 4 & 0 & 42 \\
\hline $\mathrm{A} 6$ & 19 & 29 & 25 & 1 & 1 & 5 & 0 & 80 \\
\hline $\mathrm{D} 1$ & 23 & 29 & 24 & 2 & 3 & 6 & 1 & 87 \\
\hline $\mathrm{D} 2$ & 17 & 24 & 14 & 1 & 0 & 0 & 0 & 56
\end{tabular}

Table 3.7: The average number of spike trains assigned to each muscle in the NMS model, for each subject (O2016 IEEE). The table also shows the number of units among these assigned to other muscles as well (in brackets), because the NMs model allows for one spike train to estimate the excitation of multiple muscles. This table also appeared in Kapelner, Sartori, and Farina (2016).

\begin{tabular}{c||c|c|c|c|c|c|c}
\hline & SUP & ECRL & ECU & FCR & FCU & PT & Unassigned \\
\hline \hline A1 & $14 \pm 0(0)$ & $12 \pm 1(9 \pm 3)$ & $16 \pm 1(16 \pm 1)$ & $11 \pm 3(11 \pm 3)$ & $18 \pm 0(18 \pm 0)$ & $13 \pm 0(0)$ & $5 \pm 1$ \\
\hline A2 & $5 \pm 1(1 \pm 0)$ & $18 \pm 1(14 \pm 1)$ & $12 \pm 1(12 \pm 1)$ & $12 \pm 1(11 \pm 1)$ & $9 \pm 1(9 \pm 1)$ & $4 \pm 0(1)$ & $3 \pm 1$ \\
\hline A3 & $5 \pm 1(0)$ & $23 \pm 1(23 \pm 1)$ & $21 \pm 1(20 \pm 1)$ & $21 \pm 1(17 \pm 1)$ & $16 \pm 1(14 \pm 1)$ & $5 \pm 1(0)$ & $5 \pm 1$ \\
\hline A4 & $7(0)$ & $16 \pm 0(11 \pm 5)$ & $18 \pm 0(18 \pm 1)$ & $24 \pm 5(23 \pm 5)$ & $31 \pm 1(29 \pm 1)$ & $14(0)$ & $1 \pm 1$ \\
\hline A5 & $14 \pm 0(0)$ & $5(5)$ & $13 \pm 0(11 \pm 0)$ & $5(5)$ & $11 \pm 0(11 \pm 0)$ & $3(0)$ & $5 \pm 1$ \\
\hline A6 & $10 \pm 1(1)$ & $28 \pm 2(17 \pm 6)$ & $20 \pm 1(20 \pm 1)$ & $11 \pm 6(11 \pm 6)$ & $15 \pm 1(13 \pm 1)$ & $10 \pm 3(1)$ & $15 \pm 3$ \\
\hline D1 & $8 \pm 1(0)$ & $19 \pm 1(27 \pm 2)$ & $17 \pm 1(17 \pm 1)$ & $33 \pm 2(32 \pm 2)$ & $22 \pm 1(22 \pm 1)$ & $13 \pm 1(0)$ & $13 \pm 2$ \\
\hline D2 & $8(0)$ & $19 \pm 0(19 \pm 1)$ & $20 \pm 1(20 \pm 1)$ & $17 \pm 1(17 \pm 1)$ & $19 \pm 1(18 \pm 1)$ & $5(0)$ & $2 \pm 1$
\end{tabular}

The full ANOVA reported no statistically significant difference between groups ( $\mathrm{p}=$ $0.06)$, and significant differences between the levels of Feature $(\mathrm{p}=0.01)$ and Subject $(\mathrm{p}$ $<0.01)$, as well as a two-way interaction between these two factors $(\mathrm{p}<0.001)$. Thus, subsequent one-way analyzes were performed (see 3.5.2 Methods), revealing that $\mathrm{DSC}_{\mathrm{NMS}}$ performed statistically significantly better than TD for 4 out of 6 able-bodied subjects, and for all patients (Figure 3.5.4). Moreover, there was no significant difference between $\mathrm{TD}+\mathrm{DSC}$ and $\mathrm{DSC}_{\mathrm{NMS}}$ for 3 subjects, in the other cases $\mathrm{DSC}_{\mathrm{NMS}}$ outperformed TD+DSC 
significantly. The relative performance of TD and TD+DSC was mixed, with one outperforming the other or having no significant difference for an almost equal number of subjects (Figure 3.5.4). An example of the estimation results is depicted in Figure 3.5.5.

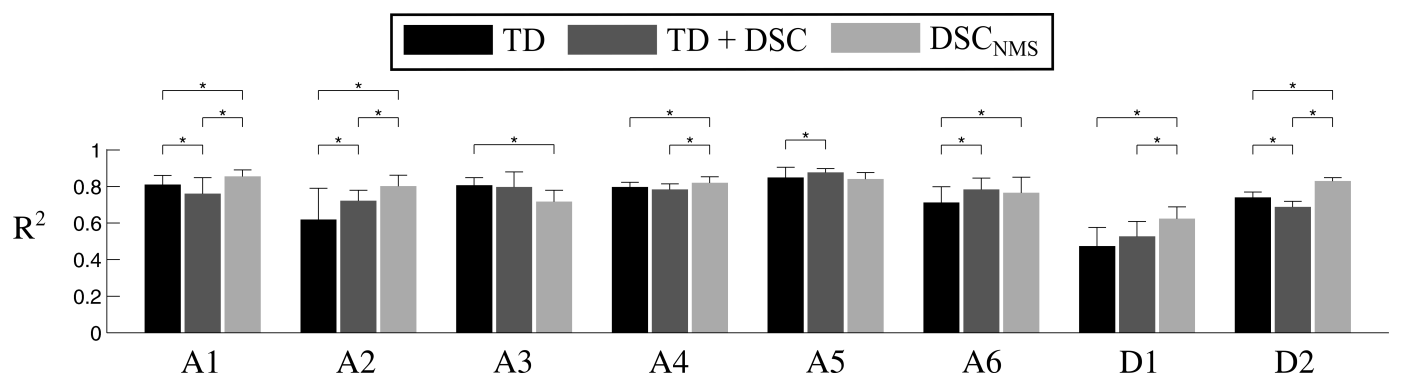

Figure 3.5.4: The performance of the investigated methods across subjects (O2016 IEEE). The TD and TD+DSC feature sets are used in ANN regression, whereas $D S C_{N M S}$ uses the proposed NMS model. This figure also appeared in Kapelner, Sartori, and Farina (2016).

\subsubsection{Discussion}

The main finding of this section was that NMS modeling driven by neural information decomposed from the surface EMG outperformed state of the art EMG-based regression. The superior performance of the proposed framework was observed in four healthy subjects and all patients, showing that using neural information is a promising framework for advanced prosthesis control.

DSC $_{\text {NMS }}$ outperforming both TD and TD+DSC in almost all cases shows that a biomechanical model was able to estimate wrist kinematics more accurately than the state of the art method, irrespective of the used feature (Figure 3.5.4).

The main advantage of $\mathrm{DSC}_{\mathrm{NMS}}$ over TD might be the explicit identification of activated muscles and thereby the DoFs to actuate. This allowed the model to avoid involuntary simultaneous activations of multiple DoFs. Nevertheless, the model estimated all muscle excitations simultaneously, and thus it is capable to also estimate simultaneous movements (Figure 3.5.5)/A). The validation of this method for simultaneous movements in multiple DoFs was, however, not performed due to the difficulties in validating such an approach in an offline context (Jiang et al. 2014).

Another explanation of the superior performance of $\mathrm{DSC}_{\mathrm{NMS}}$ could be that the proposed NMS model provides smooth estimates of the wrist kinematics, in which case a simple lowpass filter after ANN regression could provide the same results. However, the increase in performance cannot be attributed to this effect alone, since all ANN methods were low-pass filtered on the output, and $\mathrm{DSC}_{\mathrm{NMS}}$ outperformed them significantly in most cases.

The proposed NMS method outperforming TD+DSC could be explained by the difficulty of ANNs in coping with missing spikes or spike trains, which decreases the performance of TD+DSC. This might also explain the inconclusive results regarding the relative performance of TD and TD+DSC (Figure 3.5.4): subjects for which TD+DSC performed worse might have had less consistently present motor units. 
(A)
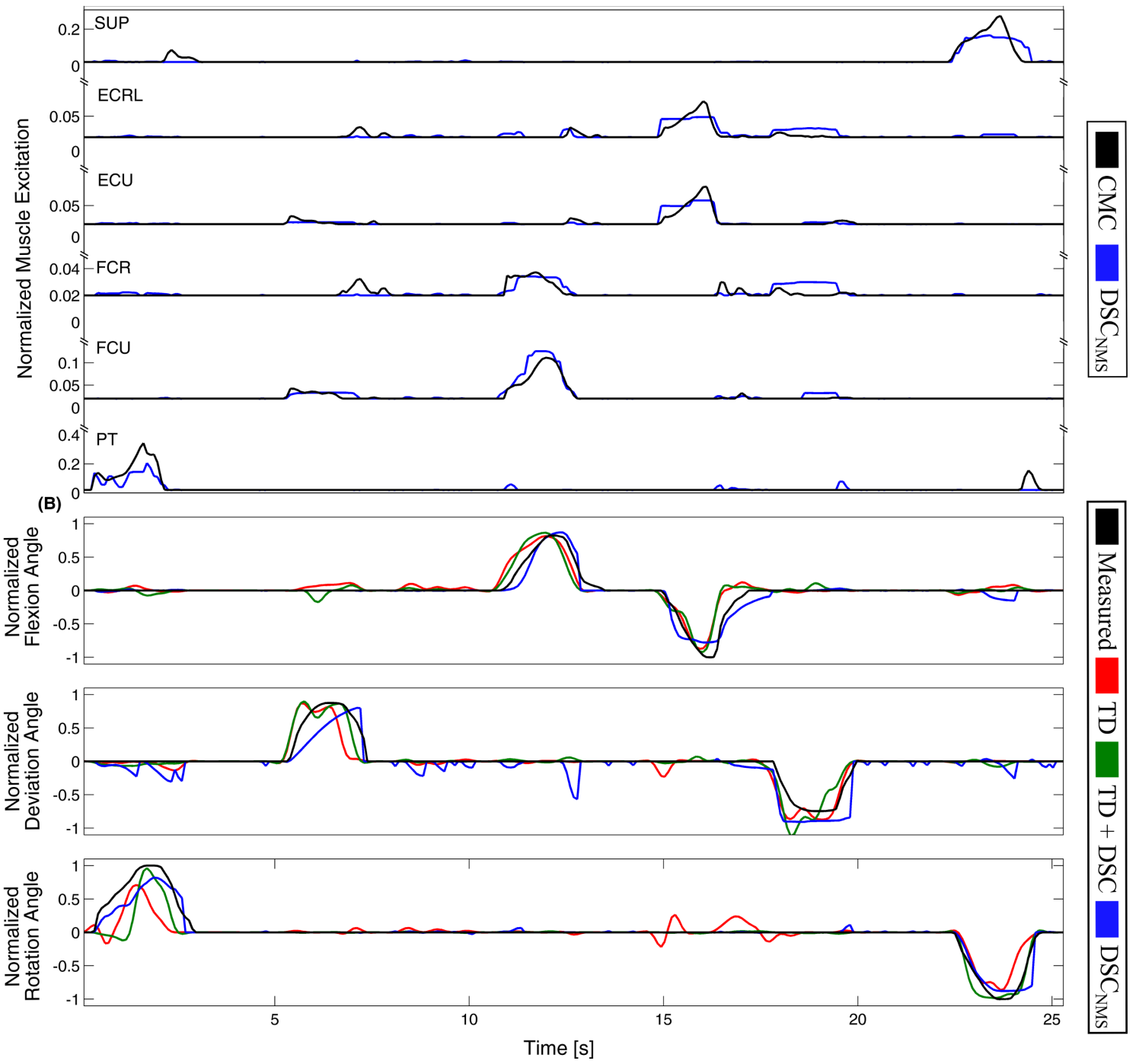

Figure 3.5.5: An example of estimation results (О2016 IEEE). (A) shows the muscle excitations estimated using neural information (blue) compared to the results of the inverse kinematics calculations using the CMC static optimization method (black). (B) shows the output of the regression methods (coloured) compared to the measured kinematics of the subject (black). This figure also appeared in Kapelner, Sartori, and Farina (2016).

The number of spike trains decomposed with this method was somewhat lower than the yield of the original CKC employed in Section 3.4 (Table 3.5). This was consistent with the results of Section 3.3, where a comparable number of spike trains was decomposed for slower wrist movements (Tables 3.3 and 3.4).

The assignment of the decomposed spike trains to muscles resulted in PT and SUP having uniquely identified motor units, whereas the other muscles shared almost all of their units (Table 3.7). This was observed due to the result of the physiological function of the muscles. Since flexors and extensors participate in both flexion/extension and deviation, a unique identification of a unit is only possible if it is detected in both DoFs. This was only observed for a small number of units in our sample (Table 3.6). It has to be noted that PT and SUP also have flexion and deviation moments physiologically, but their contribution 
was negligible for our CMC calculation.

In spite of the small number of uniquely identified motor units, the method was capable to accurately estimate the muscle excitations in a feed-forward manner (Figure 3.5.5/A). These could also be useful for several purposes other than prosthesis control, such as diagnosing or monitoring muscle weakness after amputation, or informing the training process of the prosthetic device. Such application could be reinforced by other biomechanical variables relevant to movement, such as muscle moment arms or joint moments, which are also simulated by the NMS model. Showing exactly what parameters to change in order to improve prosthesis control during training is a clear advantage of the NMS model over black-box approaches used in machine learning, and potentially reduces the required training time.

\subsubsection{Summary}

This section showed that neural information based prosthesis control is capable to outperform state of the art regression methods, which is the main outcome of this thesis. The superior performance was achieved using an NMS model consisting of two serial submodels, one for estimating muscle excitations from neural information, and another for estimating wrist kinematics based on muscle excitations by biomechanical modeling. The proposed novel prosthesis control system outperformed the state of the art regression in patients with limb deficiency as well as able-bodied controls. In addition, this framework provided additional information on muscle function that could be utilized in supporting rehabilitation or reducing the training time of prosthesis control. 


\section{GENERAL DISCUSSION}

The aim of this PhD project was to investigate whether natural, intuitive prosthesis control can be achieved by using neural information from surface EMG decomposition as an input, and to realize a proof of concept for this approach. Throughout this thesis the feasibility of the framework was demonstrated in a series of 5 studies dedicated to different aspects of this novel approach. These studies were also milestones in an iterative process that resulted in a novel regression method based on an NMS model driven by neural information, which outperformed the state of the art regression in both able-bodied subjects and subjects with limb-deficiency.

As a first step in this series of investigations I examined whether the TMR procedure causes electrophysiological changes in the reinnervated motor units, and what implications these have with respect to prosthesis control. I have found that the surface area of motor units after TMR was significantly smaller compared to motor units in the able-bodied group. In spite this high level of localization of MUAP shapes, however, motor unit surface areas as well as surface areas of motor unit groups corresponding to a single motor task were mostly overlapping.

This finding indicates that even though TMR aims to separate movements spatially in order to create EMG recording sites that are specific to a given motion, the crosstalk between these sites cannot be fully eliminated because of overlapping surface representations of motor units. This finding reveals why electrode placement in TMR patients is not straightforward, and why pattern recognition outperforms conventional control in TMR patients (Hargrove, Lock, and Simon 2013; He Huang et al. 2008). Neural information based prosthesis control could therefore offer better control performance, since overlapping MUAPs can still be separated via EMG decomposition.

This hypothesis was tested in the second section of Chapter 3, which compared neural information to time-domain features in terms of classification accuracy with pattern recognition in TMR subjects. Neural information outperformed time-domain features when the 
spike trains were pooled together based on the spatial localization of their MUAP within a given grid, highlighting the potential of this approach in prosthesis control.

However, the performance increase was not observed when all spikes from a given grid were pooled together without considering the spatial position of the MUAP. Because in TMR spatial position of a MUAP is connected to motor function, this finding shows that pooling spike trains to reduce input dimensionality for prosthesis control is only effective if the grouped motor units are functionally similar. Therefore prosthesis control methods have to be able to determine which motor units build a functional group.

It is worth noting that grouping together spike trains based on spatial position proved to be effective even though MUAP shapes were found to be largely overlapping in the first study. One explanation for this is that while individual MUAP shapes were overlapping, the overall spatial territories of all MUAP shapes belonging to each task were distinctive enough to allow the discrimination between tasks. Although spatial EMG representations of tasks can also exhibit this property, the lower performance of EMG features suggests that time-domain EMG features were not as distinctive across tasks as neural features.

Although TMR subjects can be trained to generate distinct EMG activations for each function, potentially increasing classification accuracy to the level of using neural information (Englehart and Hudgins 2003; Hargrove, Lock, and Simon 2013), the neural information based approach achieved an almost perfect separation of classes without prior training. This suggests that neural information based prosthesis control can indeed provide more natural control with less training required.

To translate the previous findings to regression methods and transradial amputees, the third study was designed to investigate whether EMG decomposition provides enough information for prosthesis control in non-isometric voluntary contractions, in terms of the number of spikes and the repeatability of the decomposition. In both able-bodied subjects and subjects with limb deficiency, the decomposition of the EMG signals recorded during three DoF wrist motions yielded on average 16 spike trains per motor task, 6 of which were present in all repetitions of a given task, demonstrating the feasibility of EMG decomposition for regression.

Section 3.3 also found that the majority of the identified motor units were recruited above $20 \%$ of the maximal range of movement. This shows that motor units at low wrist angles were not always detected by the decomposition algorithm and that methods dedicated to non-isometric contractions would be needed to fully realize the potential of this framework.

The fourth study described the structure of a linear regression method based on neural information and validated the methods by comparing its performance with that of timedomain EMG features. The developed novel regression method took the experiences of the previous studies into account. Firstly, because spike trains are rarely present at low joint angles, according to Section 3.3.4, the raw EMG was included in the feature set along with the spike trains to compensate for the missing information. Secondly, to compensate for not consistently present spikes, a robust regression method was chosen to estimate kinematics. Lastly, since Section 3.2.4 highlighted the importance of the grouping of motor units, the 
method employed a grouping scheme for spike trains inspired by motor unit physiology.

The method was validated in three degrees of freedom contractions of the wrist in varying speeds and movement ranges. It outperformed linear regression using EMG features in all conditions, for both able-bodied subjects and subjects with limb deficiency. For the superior performance over the full range of movements the inclusion of the raw EMG in the feature set was necessary, as expected based on the findings of Section 3.3.

Section 3.4 also revealed that the lack of decomposed units below $20 \%$ of the maximal range of movement was not observed when $20 \%$ was the largest normalized angle reached within the decomposed interval. Thus, future decomposition methods dedicated to nonisometric contractions will have to compensate for the presence of high EMG activity at large wrist angles that potentially suppresses MUAPs at low force levels.

In the final section of Chapter 3 the natural and intuitive nature of neural information was fully exploited by using an NMS model driven by neural information to estimate wrist kinematics. The validation of the model showed that this approach was capable to outperform state of the art nonlinear regression in both able-bodied subjects and patients with limb deficiency, and accurately estimate muscle excitations during movement. Moreover, the detailed biomechanical information provided by the method allows for supporting the rehabilitation and training process.

The results of the above studies strongly suggest that prosthesis control by using neural information acquired thorough surface EMG decomposition is possible in healthy subjects, subjects with limb deficiency and TMR subjects, with a higher performance than traditional myoelectric control methods in all subject groups. This was shown for the first time by this thesis.

The use of the control approaches introduced in this thesis is not limited to neural information acquired from the surface EMG. They are suitable for any method that is capable to extract spike trains of motor unit populations, including future online EMG decomposition algorithms of surface or intramuscular EMG (Negro et al. 2016), as well as other methods such as peripheral nerve electrodes (Boretius et al. 2010; Lago et al. 2005; Micera et al. 2008).

As a consequence of the novelty of the approach introduced by the thesis, there are some limitations that need to be addressed. These arise because there is no real-time EMG decomposition method available to date, even though such an algorithm has been shown to be feasible (Glaser, Holobar, and Zazula 2013).

Firstly, it was not possible to assess the online performance of the proposed methods. This would be relevant because the difference in offline performance does not necessarily translate to a difference in performance during an online test (Jiang et al. 2014). However, the control approach proposed by this thesis might still be more intuitive to use, and require less training - or retraining over time - than current EMG-based methods, as suggested by Section 3.2.4.

The development of online decomposition would also allow the evaluation of simultaneous control of multiple DoFs. Although all methods presented in this thesis are capable to estimate simultaneous movements in multiple DoFs without modification of the algo- 
rithms, validation trials in this context were not in the scope of this thesis. This is because the use of simultaneous control by subjects is specific to the task and to the used control method (Smith, Kuiken, and Hargrove 2016). Thus, differences in simultaneous control performance in an (artificial) offline task would not necessarily be relevant during natural tasks.

Moreover, since Section 3.3 showed that EMG decomposition might fail to detect motor units at small wrist angles, further decrease in decomposition performance is expected during concurrent activations of multiple DoFs. EMG decomposition in these circumstances would require methods designed to cope with MUAP changes due to significant muscle movement under the skin.

Nevertheless, the technological limitation of offline decomposition is likely to be overcome by the increase of computational power, and this thesis showed that realizing online EMG decomposition specific to non-isometric conditions is a promising avenue of further research with a possible application in advanced prosthesis control.

In conclusion, this thesis has investigated several aspects of prosthesis control based on neural information decomposed from the surface EMG, in several subject groups. It revealed insights in the physiological background of such an approach in TMR, the information content of EMG decomposition with respect to control, and the performance of several implementations of the control approach as a proof of concept. The thesis has demonstrated that natural, intuitive prosthesis control can indeed be achieved using neural information obtained with available surface EMG decomposition methods, and online EMG decomposition would merit further investigation in order to assess the online control performance and to provide better functionality for prosthesis users with a natural and intuitive interface. 


\section{BIBLIOGRAPHY}

Amsüss, S. et al. (2015). "A multi-class proportional myocontrol algorithm for upper limb prosthesis control: validation in real-life scenarios on amputees". In: Neural Systems and Rehabilitation Engineering, IEEE Transactions on 23.5, pp. 827-836.

Amsüss, S. et al. (2016). "Context-Dependent Upper Limb Prosthesis Control for Natural and Robust Use". In: IEEE Transactions on Neural Systems and Rehabilitation Engineering 24.7, pp. 744-753.

Aszmann, O., H Dietl, and M Frey (2008). "[Selective nerve transfers to improve the control of myoelectrical arm prostheses]". German. In: Handchirurgie, Mikrochirurgie, plastische Chirurgie 40.1, pp. 60-65.

Atroshi, I. and H.-E. Rosberg (2001). "Epidemiology of amputations and severe injuries of the hand." In: Hand clinics 17.3, pp. 343-50.

Atzori, M. and H. Müller (2015). "Control Capabilities of Myoelectric Robotic Prostheses by Hand Amputees: A Scientific Research and Market Overview". In: Frontiers in systems neuroscience 9 .

Basmajian, J. and C. J. De Luca (1985). Muscles Alive. Baltimore, MD: Williams and Wilkins.

Biddiss, E. and T. Chau (2007a). "Upper-limb prosthetics: critical factors in device abandonment". In: American Journal of Physical Medicine E Rehabilitation 86.12, pp. 977987.

Biddiss, E. A. and T. T. Chau (2007b). "Upper limb prosthesis use and abandonment: a survey of the last 25 years". In: Prosthetics and orthotics international 31.3, pp. 236257.

Bigland-Ritchie, B. et al. (1983). "Changes in motoneurone firing rates during sustained maximal voluntary contractions." In: The Journal of Physiology 340, p. 335.

Bodine-Fowler, S. et al. (1990). "Spatial distribution of muscle fibers within the territory of a motor unit". In: Muscle \& nerve 13.12, pp. 1133-1145.

Boretius, T. et al. (2010). "A transverse intrafascicular multichannel electrode (TIME) to interface with the peripheral nerve". In: Biosensors and Bioelectronics 26.1, pp. 62-69. 
Buchanan, T. S. et al. (2004). "Neuromusculoskeletal modeling: estimation of muscle forces and joint moments and movements from measurements of neural command". In: Journal of applied biomechanics 20.4, p. 367.

Buller, A., J. Eccles, and R. M. Eccles (1960). "Interactions between motoneurones and muscles in respect of the characteristic speeds of their responses". In: The Journal of physiology 150.2, p. 417.

Chang, C.-C. and C.-J. Lin (2011). "LIBSVM: a library for support vector machines". In: ACM Transactions on Intelligent Systems and Technology (TIST) 2.3, p. 27.

Chen, A. et al. (2013). "Cortical motor activity and reorganization following upperlimb amputation and subsequent targeted reinnervation". In: NeuroImage: Clinical 3, pp. 498-506.

Chen, M. and P. Zhou (2016). "A novel framework based on FastICA for high density surface EMG decomposition”. In: IEEE Transactions of Neural Systems Rehabilitation Engineering 24.1, pp. 117-127.

Chu, J. et al. (2012). "Improvement of signal-to-interference ratio and signal-to-noise ratio in nerve cuff electrode systems". In: Physiological Measurements 33.6, p. 943.

Chu, J.-U., I. Moon, and M.-S. Mun (2006). "A real-time EMG pattern recognition system based on linear-nonlinear feature projection for a multifunction myoelectric hand". In: Biomedical Engineering, IEEE Transactions on 53.11, pp. 2232-2239.

Colombini, B. et al. (2007). "Crossbridge properties during force enhancement by slow stretching in single intact frog muscle fibres". In: The Journal of physiology 585.2, pp. 607-615.

Cutsem, M., J. Duchateau, and K. Hainaut (1998). "Changes in single motor unit behaviour contribute to the increase in contraction speed after dynamic training in humans". In: The Journal of physiology 513.1, pp. 295-305.

Dario Farina et al. (2016). "Man-Machine Interfacing With Discharge Timings of Spinal Motor Neurons After Targeted Muscle Reinnervation". In: Nature Biomedical Engineering. Submitted for publication.

d'Avella, A. et al. (2006). "Control of fast-reaching movements by muscle synergy combinations". In: The Journal of neuroscience 26.30, pp. 7791-7810.

Day, S. (2002). "Important factors in surface EMG measurement". In: Bortec Biomedical Ltd publishers, pp. 1-17.

De Luca, C. J. et al. (1982). "Behaviour of human motor units in different muscles during linearly varying contractions". In: The Journal of Physiology 329.1, pp. 113-128.

De Luca, C. J. (1979). "Physiology and Mathematics of Myoelectric Signals". In: IEEE Transactions on Biomedical Engineering 26.6, pp. 313-325.

De Luca, C. J. and P. Contessa (2012). "Hierarchical control of motor units in voluntary contractions". In: Journal of neurophysiology 107.1, pp. 178-195.

De Luca, C. J. and Z. Erim (1994). "Common drive of motor units in regulation of muscle force". In: Trends in neurosciences 17.7, pp. 299-305.

- (2002). "Common drive in motor units of a synergistic muscle pair". In: Journal of neurophysiology 87.4, pp. 2200-2204. 
De Luca, C. J. and R. Merletti (1988). "Surface myoelectric signal cross-talk among muscles of the leg". In: Electroencephalography and clinical neurophysiology 69.6, pp. 568575 .

De Luca, C. J. and S. H. Nawab (2011). "Reply to Farina and Enoka: the reconstruct-andtest approach is the most appropriate validation for surface EMG signal decomposition to date". In: Journal of neurophysiology 105.2, pp. 983-984.

De Luca, C. J. et al. (2015). "Decomposition of surface EMG signals from cyclic dynamic contractions". In: Journal of neurophysiology 113.6, pp. 1941-1951.

De Zepetnek, J. T. et al. (1992). "Innervation ratio is an important determinant of force in normal and reinnervated rat tibialis anterior muscles". In: Journal of neurophysiology 67.5 , pp. $1385-1403$.

Delp, S. L. et al. (2007). "OpenSim: open-source software to create and analyze dynamic simulations of movement". In: Biomedical Engineering, IEEE Transactions on 54.11, pp. $1940-1950$.

Demet, K. et al. (2003). "Health related quality of life and related factors in 539 persons with amputation of upper and lower limb". In: Disability and rehabilitation 25.9, pp. $480-486$.

Desmedt, J. E. and E. Godaux (1977). "Fast motor units are not preferentially activated in rapid voluntary contractions in man". In: Nature 267, pp. 717-719.

Desnedt, H. E. and E. Gidaux (1981). "Spinal motoneuron recruitment in man: rank deordering with direction but not with speed of voluntary movement". eng. In: Science 214.4523, pp. 933-936.

Dhillon, G. S. and K. W. Horch (2005). "Direct neural sensory feedback and control of a prosthetic arm". In: Neural Systems and Rehabilitation Engineering, IEEE Transactions on 13.4 , pp. 468-472.

Duchateau, J. and R. M. Enoka (2008). "Neural control of shortening and lengthening contractions: influence of task constraints". eng. In: The Journal of Physiology 586.24, pp. 5853-5864.

Dumanian, G. A. et al. (2009). "Targeted reinnervation for transhumeral amputees: current surgical technique and update on results". In: Plastic and reconstructive surgery 124.3, pp. 863-869.

Durance, J. P. and B. J. O'shea (1988). "Upper limb amputees: a clinic profile". In: International disability studies 10.2, pp. 68-72.

ElKoura, G. and K. Singh (2003). "Handrix: animating the human hand". In: Proceedings of the 2003 ACM SIGGRAPH/Eurographics symposium on Computer animation. Eurographics Association, pp. 110-119.

Englehart, K. and B. Hudgins (2003). "A Robust, Real-Time Control Scheme for Multifunction Myoelectric Control". In: IEEE Transacions on Biomedical Engineering 50.7, pp. 848-854.

Enoka, R. M. (2008). Neuromechanics of human movement. 4th ed. Champaign: Human kinetics. 
Farina, D. et al. (2014). "Noninvasive, Accurate Assessment of the Behavior of Representative Populations of Motor Units in Targeted Reinnervated Muscles". In: Neural Systems and Rehabilitation Engineering, IEEE Transactions on 22.4, pp. 810-819.

Farina, D. and R. M. Enoka (2011). "Surface EMG decomposition requires an appropriate validation". In: Journal of neurophysiology 105.2, pp. 981-982.

Farina, D. and R. Merletti (2001). "A novel approach for precise simulation of the EMG signal detected by surface electrodes". In: IEEE Transactions on Biomedical Engineering 48.6, pp. 637-646.

Farina, D., R. Merletti, and R. M. Enoka (2004). "The extraction of neural strategies from the surface EMG". In: Journal of Applied Physiology 96.4, pp. 1486-1495.

- (2015). Reply to De Luca, Nawab, and Kline: The proposed method to validate surface EMG signal decomposition remains problematic. Vol. 118. 8. Am Physiological Soc.

Farina, D., F. Negro, and J. L. Dideriksen (2014). "The effective neural drive to muscles is the common synaptic input to motor neurons". In: The Journal of physiology 592.16, pp. 3427-3441.

Farina, D. et al. (2008). "Detecting the unique representation of motor-unit action potentials in the surface electromyogram". In: Journal of Neurophysiology 100.3, pp. 12231233.

Farina, D. et al. (2010). "Decoding the neural drive to muscles from the surface electromyogram". In: Clinical Neurophysiology 121.10, pp. 1616-1623.

Filippi, G. M. and D. Troian (1994). "Relations among motor unit types, generated forces and muscle length in single motor units of anaesthetized cat peroneus longus muscle". In: Experimental brain research 101.3, pp. 406-414.

Flor, H. et al. (1995). "Phantom-limb pain as a perceptual correlate of cortical reorganization following arm amputation". In: Nature 375.6531, pp. 482-484.

Foehring, R. C., G. W. Sypert, and J. B. Munson (1987). "Motor-unit properties following cross-reinnervation of cat lateral gastrocnemius and soleus muscles with medial gastrocnemius nerve. II. Influence of muscle on motoneurons." In: Journal of Neurophysiology 57 (4), pp. 1210-26.

Fougner, A. et al. (2012). "Control of upper limb prostheses: terminology and proportional myoelectric control - a review". In: Neural Systems and Rehabilitation Engineering, IEEE Transactions on 20.5, pp. 663-677.

Fuglevand, A. J., D. A. Winter, and A. E. Patla (1993). "Models of recruitment and rate coding organization in motor-unit pools". In: Journal of neurophysiology 70.6, pp. 2470-2488.

Fuglevand, A. J. et al. (1992). "Detection of motor unit action potentials with surface electrodes: influence of electrode size and spacing". In: Biological cybernetics 67.2, pp. $143-153$.

Gaine, W. J., C. Smart, and M. Bransby-Zachary (1997). "Upper limb traumatic amputees Review of prosthetic use". In: Journal of Hand Surgery (British and European Volume) 22.1 , pp. $73-76$. 
Gak, J. et al. (2008). "On the reduction of thermal and flicker noise in ENG signal recording amplifiers". In: Analog Integrated Circuits and Signal Processing 57.1-2, pp. 39-48.

Glaser, V., A. Holobar, and D. Zazula (2013). "Real-Time Motor Unit Identification From High-Density Surface EMG". In: IEEE Transactions on Neural Systems and Rehabilitation Engineering 21.6, pp. 949-957.

Gordon, T. and M. C. Pattullo (1993). "Plasticity of muscle fiber and motor unit types." In: Exercise and sport sciences reviews 21.1, pp. 331-362.

Hahne, J. M. et al. (2012). "Simultaneous and proportional control of 2D wrist movements with myoelectric signals". In: Machine Learning for Signal Processing (MLSP), 2012 IEEE International Workshop on. IEEE, pp. 1-6.

Hahne, J. et al. (2014). "Linear and Nonlinear Regression Techniques for Simultaneous and Proportional Myoelectric Control". In: Neural Systems and Rehabilitation Engineering, IEEE Transactions on 22.2, pp. 269-279.

Hamid R. Marateb et al. (2011). "Accuracy assessment of CKC high-density surface EMG decomposition in biceps femoris muscle". In: J Neural Eng. 8.6.

Hargrove, L. et al. (2009). "The effect of ECG interference on pattern-recognition-based myoelectric control for targeted muscle reinnervated patients". In: Biomedical Engineering, IEEE Transactions on 56.9, pp. 2197-2201.

Hargrove, L. J., B. A. Lock, and A. M. Simon (2013). "Pattern recognition control outperforms conventional myoelectric control in upper limb patients with targeted muscle reinnervation". In: Engineering in Medicine and Biology Society (EMBC), 2013 35th Annual International Conference of the IEEE, pp. 1599-1602.

He Huang et al. (2008). "An Analysis of EMG Electrode Configuration for Targeted Muscle Reinnervation Based Neural Machine Interface". In: IEEE Transactions on Neural Systems and Rehabilitation Engineering 16.1, pp. 37-45.

Hebert, J. S. et al. (2014). "Novel targeted sensory reinnervation technique to restore functional hand sensation after transhumeral amputation". In: Neural Systems and Rehabilitation Engineering, IEEE Transactions on 22.4, pp. 765-773.

Henneman, E. (1957). "Relation between size of neurons and their susceptibility to discharge". In: Science 126.3287, pp. 1345-1347.

Henneman, E., G. Somjen, and D. O. Carpenter (1965). "Excitability and inhibitibility of motoneurons of different sizes". In: Journal of neurophysiology 28.3, pp. 599-620.

Hodson-Tole, E. F. and J. M. Wakeling (2009). "Motor unit recruitment for dynamic tasks: current understanding and future directions". en. In: Journal of Comparative Physiology B 179.1, pp. 57-66.

Holobar, A., M. A. Minetto, and D. Farina (2014). "Accurate identification of motor unit discharge patterns from high-density surface EMG and validation with a novel signalbased performance metric". In: Journal of Neural Engineering 11.1, p. 016008.

Holobar, A. et al. (2011). "Identification of Motor Unit Discharge Patterns from HighDensity Surface EMG during High Contraction Levels". en. In: 5th European Conference of the International Federation for Medical and Biological Engineering. IFMBE Proceedings 37, pp. 1165-1168. 
Holobar, A. and D. Zazula (2007). "Multichannel Blind Source Separation Using Convolution Kernel Compensation". In: IEEE Transactions on Signal Processing 55.9, pp. 4487-4496.

Holobar, A. et al. (2009). "Estimating motor unit discharge patterns from high-density surface electromyogram". In: Clinical Neurophysiology 120.3, pp. 551-62.

Hotelling, H. (1933). "Analysis of a complex of statistical variables into principal components." In: Journal of educational psychology 24.6, p. 417.

Hu, X., W. Z. Rymer, and N. L. Suresh (2013). "Motor unit pool organization examined via spike-triggered averaging of the surface electromyogram". In: Journal of neurophysiology 110.5, pp. 1205-1220.

Huang, H. et al. (2009). "Spatial Filtering Improves EMG Classification Accuracy Following Targeted Muscle Reinnervation". en. In: Annals of Biomedical Engineering 37.9, pp. 1849-1857.

Hudgins, B., P. Parker, and R. N. Scott (1993). "A new strategy for multifunction myoelectric control". In: Biomedical Engineering, IEEE Transactions on 40.1, pp. 8294.

Ingraham, K. A. et al. (2015). "Nonlinear mappings between discrete and simultaneous motions to decrease training burden of simultaneous pattern recognition myoelectric control". In: Engineering in Medicine and Biology Society (EMBC), 2015 37th Annual International Conference of the IEEE. IEEE, pp. 1675-1678.

Jiang, N. (2009). "Extracting force functions from the multi-channel surface electromyographic signal." PhD. University of New Brunswick (Canada).

Jiang, N. et al. (2012a). "EMG-based simultaneous and proportional estimation of wrist/hand kinematics in uni-lateral trans-radial amputees". In: J Neuroeng Rehabil 9, pp. $42-$ 53.

Jiang, N. et al. (2012b). "Myoelectric Control of Artificial Limbs - Is There a Need to Change Focus?" In: IEEE Signal Processing Magazine 29.5, pp. 152, 148-150.

Jiang, N. et al. (2014). "Is accurate mapping of emg signals on kinematics needed for precise online myoelectric control?" In: Neural Systems and Rehabilitation Engineering, IEEE Transactions on 22.3 , pp. 549-558.

Kanda, K. and J. E. Desmedt (1983). "Cutaneous facilitation of large motor units and motor control of human fingers in precision grip". eng. In: Advances in Neurology 39, pp. 253-261.

Kanda, K. and K. Hashizume (1992). "Factors causing difference in force output among motor units in the rat medial gastrocnemius muscle." In: The Journal of physiology 448, p. 677.

Kapelner, T., N. Jiang, and D. Farina (2016). "Regression using motor unit spike trains for predicting wrist kinematics in hand prostheses". In: Journal of Neural Engineering. Submitted for publication.

Kapelner, T., F. Negro, and D. Farina (2016). "Decoding the behavior of motor units from forearm muscles during dynamic contractions: perspectives for myoelectric con- 
trol". In: Transactions on Neural Systems 83 Rehabilitation Engineering. Submitted for publication.

Kapelner, T., M. Sartori, and D. Farina (2016). "A neuromusculoskeletal modeling approach for myoelectric control". In: Transactions on Neural Systems $\& 3$ Rehabilitation Engineering. In Preparation.

Kapelner, T. et al. (2015). "Classification of motor unit activity following targeted muscle reinnervation". In: 7th International Conference on Neural Engineering. Montpellier: IEEE/EMBS, pp. 652-654.

Kapelner, T. et al. (2016). "Motor Unit Characteristics after Targeted Muscle Reinnervation". In: PLOS ONE 11.2, e0149772.

Kossev, A. and P. Christova (1998). "Discharge pattern of human motor units during dynamic concentric and eccentric contractions". In: Electroencephalography and Clinical Neurophysiology/Electromyography and Motor Control 109.3, pp. 245-255.

Kugelberg, E., L. Edström, and M. Abbruzzese (1970). "Mapping of motor units in experimentally reinnervated rat muscle Interpretation of histochemical and atrophic fibre patterns in neurogenic lesions". In: Journal of Neurology, Neurosurgery $\& 5$ Psychiatry 33.3, pp. 319-329.

Kuiken, T. A. et al. (2004). "The use of targeted muscle reinnervation for improved myoelectric prosthesis control in a bilateral shoulder disarticulation amputee". In: Prosthetics and Orthotics International 28.3, pp. 245-253.

Kuiken TA et al. (2009). "Targeted muscle reinnervation for real-time myoelectric control of multifunction artificial arms". In: JAMA 301.6, pp. 619-628.

Kukulka, C. G. and H. P. Clamann (1981). "Comparison of the recruitment and discharge properties of motor units in human brachial biceps and adductor pollicis during isometric contractions". In: Brain research 219.1, pp. 45-55.

Lago, N. et al. (2005). "Long term assessment of axonal regeneration through polyimide regenerative electrodes to interface the peripheral nerve". In: Biomaterials 26.14, pp. 2021-2031.

Laine, C. M., U. Yavuz, and D. Farina (2014). "Task-related changes in sensorimotor integration influence the common synaptic input to motor neurones". In: Acta Physiologica 211.1, pp. 229-239.

Lake, C. and J. M. Miguelez (2003). "Comparative analysis of microprocessors in upper limb prosthetics". In: JPO: Journal of Prosthetics and Orthotics 15.2, pp. 48-63.

Lawrence, S. M. et al. (2004). "Acute peripheral nerve recording characteristics of polymerbased longitudinal intrafascicular electrodes". In: Neural Systems and Rehabilitation Engineering, IEEE Transactions on 12.3, pp. 345-348.

Lemay, M. A. and P. E. Crago (1996). "A dynamic model for simulating movements of the elbow, forearm, and wrist". In: Journal of biomechanics 29.10, pp. 1319-1330.

Li, G., A. E. Schultz, and T. A. Kuiken (2010). "Quantifying Pattern Recognition - Based Myoelectric Control of Multifunctional Transradial Prostheses". In: IEEE transactions on neural systems and rehabilitation engineering: a publication of the IEEE Engineering in Medicine and Biology Society 18.2, pp. 185-192. 
Lieber, R. L. and S. R. Ward (2011). "Skeletal muscle design to meet functional demands". In: Philosophical Transactions of the Royal Society of London B: Biological Sciences 366.1570, pp. 1466-1476.

Lloyd, D. G. et al. (2008). "Neuromusculoskeletal modelling and simulation of tissue load in the lower extremities". In: Handbook of Biomechanics and Human Movement Science. New York: Routledge, pp. 3-17.

Majors, B. J. and J. S. Wayne (2011). "Development and validation of a computational model for investigation of wrist biomechanics". In: Annals of biomedical engineering 39.11, pp. 2807-2815.

Marasco, P. D., A. E. Schultz, and T. A. Kuiken (2009). "Sensory capacity of reinnervated skin after redirection of amputated upper limb nerves to the chest". In: Brain 132.6, pp. 1441-1448.

Masakado, Y. et al. (1995). "Motor unit firing behavior in slow and fast contractions of the first dorsal interosseous muscle of healthy men". In: Electroencephalography and Clinical Neurophysiology/Electromyography and Motor Control 97.6, pp. 290-295.

Micera, S. et al. (2008). "On the Use of Longitudinal Intrafascicular Peripheral Interfaces for the Control of Cybernetic Hand Prostheses in Amputees". In: IEEE Transactions on Neural Systems and Rehabilitation Engineering 16.5, pp. 453-472.

Moritz, C. T. et al. (2005). "Discharge rate variability influences the variation in force fluctuations across the working range of a hand muscle". In: Journal of neurophysiology 93.5, pp. 2449-2459.

Navarro, X. et al. (2005). "A critical review of interfaces with the peripheral nervous system for the control of neuroprostheses and hybrid bionic systems". In: Journal of the Peripheral Nervous System 10.3, pp. 229-258.

Nawab, S. H., S.-S. Chang, and C. J. De Luca (2010). "High-yield decomposition of surface EMG signals". en. In: Clinical Neurophysiology 121.10, pp. 1602-1615.

Nazarpour, K. et al. (2013). "A note on the probability distribution function of the surface electromyogram signal". In: Brain research bulletin 90, pp. 88-91.

Negro, F., A. Holobar, and D. Farina (2009). "Fluctuations in isometric muscle force can be described by one linear projection of low-frequency components of motor unit discharge rates". In: The Journal of physiology 587.24, pp. 5925-5938.

Negro, F. et al. (2016). "Multi-channel intramuscular and surface EMG decomposition by convolutive blind source separation". In: Journal of Neural Engineering 13.2, p. 026027.

Nerlich, A. G. et al. (2000). "Ancient Egyptian prosthesis of the big toe". In: The Lancet 356.9248, pp. 2176-2179.

Nielsen, J. L. et al. (2011). "Simultaneous and proportional force estimation for multifunction myoelectric prostheses using mirrored bilateral training". In: Biomedical Engineering, IEEE Transactions on 58.3, pp. 681-688.

Ohad Gal (2014). fit_ellipse.m. https://www.mathworks.com/matlabcentral/fileexchange/3215fit-ellipse; Last Accessed in October 2014. 
Østlie, K. et al. (2011). "Adult acquired major upper limb amputation in Norway: prevalence, demographic features and amputation specific features. A population-based survey". In: Disability and rehabilitation 33.17-18, pp. 1636-1649.

Østlie, K. et al. (2012). "Prosthesis rejection in acquired major upper-limb amputees: a population-based survey". In: Disability and Rehabilitation: Assistive Technology 7.4, pp. 294-303.

Penfield, W. and E. Boldrey (1937). "Somatic motor and sensory representation in the cerebral cortex of man as studied by electrical stimulation." In: Brain: A journal of neurology.

Person, K. (1901). On Lines and Planes of Closest Fit to System of Points in Space. Philiosophical Magazine, 2, 559-572.

Petit, J., M.-A. Giroux-Metges, and M. Gioux (2003). "Power developed by motor units of the peroneus tertius muscle of the cat". In: Journal of neurophysiology 90.5, pp. 30953104.

Riley, Z. A. et al. (2008). "Motor unit recruitment and bursts of activity in the surface electromyogram during a sustained contraction". en. In: Muscle $\& 3$ Nerve 37.6, pp. 745753.

Roberto Merletti and Dario Farina, eds. (2016). Surface Electromyography: Physiology, Engineering and Applications. Wiley-IEEE Press.

Rodri, F. J. et al. (2000). "Polyimide cuff electrodes for peripheral nerve stimulation". In: Journal of neuroscience methods 98.2, pp. 105-118.

Roeleveld, K. et al. (1997). "Volume conduction models for surface EMG; confrontation with measurements". In: Journal of Electromyography and Kinesiology 7.4, pp. 221 -232 .

Sandercock, T. G. et al. (1985). "Single motor unit and fiber action potentials during fatigue". In: Journal of Applied Physiology 58.4, pp. 1073-1079.

Sartori, M. and D. Farina (2016). "Neural Data-driven Musculoskeletal Modeling for Personalized Neurorehabilitation Technologies". In: IEEE Transactions on Biomedical Engineering 63.5, pp. 879-893.

Sartori, M., D. Farina, and D. G. Lloyd (2014). "Hybrid neuromusculoskeletal modeling to best track joint moments using a balance between muscle excitations derived from electromyograms and optimization". In: Journal of biomechanics 47.15, pp. 3613-3621.

Saul, K. R. et al. (2015). "Benchmarking of dynamic simulation predictions in two software platforms using an upper limb musculoskeletal model". In: Computer methods in biomechanics and biomedical engineering 18.13, pp. 1445-1458.

Scheme, E. et al. (2013). "Motion Normalized Proportional Control for Improved Pattern Recognition Based Myoelectric Control". In: IEEE Transactions on Neural Systems and Rehabilitation Engineering 22.1, pp. 149-157.

Schulz, S. (2011). "First experiences with the vincent hand". In: Myoelectric Symposium.

Simon, A. M. et al. (2011). "A decision-based velocity ramp for minimizing the effect of misclassifications during real-time pattern recognition control". In: Biomedical Engineering, IEEE Transactions on 58.8, pp. 2360-2368. 
Smith, L., T. Kuiken, and L. Hargrove (2016). "Myoelectric control system and taskspecific characteristics affect voluntary use of simultaneous control". In: IEEE Transactions on Neural Systems and Rehabilitation Engineering 24.1, pp. 109-116.

Smith, L. H. and L. J. Hargrove (2013). "Intramuscular EMG after targeted muscle reinnervation for pattern recognition control of myoelectric prostheses". In: Neural Engineering (NER), 2013 6th International IEEE/EMBS Conference on. IEEE, pp. 11551158 .

Smith, L. H., T. A. Kuiken, and L. J. Hargrove (2014). "Real-time simultaneous and proportional myoelectric control using intramuscular EMG". In: Journal of Neural Engineering 11.6, p. 066013.

Smith, L. H. et al. (2011). "Determining the optimal window length for pattern recognitionbased myoelectric control: Balancing the competing effects of classification error and controller delay". In: Neural Systems and Rehabilitation Engineering, IEEE Transactions on 19.2 , pp. 186-192.

Solomonow, M. et al. (1994). "Surface and wire EMG crosstalk in neighbouring muscles". en. In: Journal of Electromyography and Kinesiology 4.3, pp. 131-142.

Sreter, F., A. Luff, and J Gergely (1975). "Effect of cross-reinnervation on physiological parameters and on properties of myosin and sarcoplasmic reticulum of fast and slow muscles of the rabbit." In: The Journal of general physiology 66.6, pp. 811-821.

Thelen, D. G., F. C. Anderson, and S. L. Delp (2003). "Generating dynamic simulations of movement using computed muscle control". In: Journal of biomechanics 36.3, pp. 321328.

Tillander, J. et al. (2010). "Osseointegrated Titanium Implants for Limb Prostheses Attachments: Infectious Complications". en. In: Clinical Orthopaedics and Related Research Â̋ 468.10, pp. 2781-2788.

Troccaz, J. and C. Connolly (2008). "Prosthetic hands from touch bionics". In: Industrial Robot: An International Journal 35.4, pp. 290-293.

Tyler, D. J. and D. M. Durand (2002). "Functionally selective peripheral nerve stimulation with a flat interface nerve electrode". In: Neural Systems and Rehabilitation Engineering, IEEE Transactions on 10.4, pp. 294-303.

Van Der Niet Otr, O. et al. (2010). "The i-LIMB hand and the DMC plus hand compared: a case report". In: Prosthetics and orthotics international 34.2, pp. 216-220.

Wright, T. W., A. D. Hagen, and M. B. Wood (1995). "Prosthetic usage in major upper extremity amputations". In: The Journal of hand surgery 20.4, pp. 619-622.

Young, A. J. et al. (2013). "Classification of simultaneous movements using surface EMG pattern recognition". In: Biomedical Engineering, IEEE Transactions on 60.5, pp. 12501258 .

- (2014). "A comparison of the real-time controllability of pattern recognition to conventional myoelectric control for discrete and simultaneous movements". In: Journal of neuroengineering and rehabilitation 11.1, p. 1. 
Ziegler-Graham, K. et al. (2008). "Estimating the prevalence of limb loss in the United States: 2005 to 2050". In: Archives of physical medicine and rehabilitation 89.3, pp. 422429 . 


\section{A EMG decomposition using the CKC method}

The CKC algorithm is based on (2.1) extended for multichannel EMG signals expressed in discrete time:

$$
x_{j}[n]=\sum_{i=1}^{N} \sum_{l=0}^{L-1} h_{i j}[l] s_{i j}[n-l],
$$

where $x_{j}$ is the $j$-th channel of the EMG signal, $N$ is the number of motor units observed by all electrodes, $h_{i j}$ is the $i$-th MUAP shape as detected by electrode $j$, also referred to as convolution kernels in this context, $s_{i}$ is the spike train containing consecutive discharges of the $i$-th motor unit, and $L$ is the length of the discrete-time convolution window, or equivalently the length of the longest MUAP shape $h_{i j}$.

We can write (A.1) in matrix form extending the vector of spike trains by $L-1$ observations to $\boldsymbol{s}[n]=\left[s_{1}[n] s_{1}[n-1] \ldots s_{1}[n-L+1] s_{2}[n] \ldots s_{N}[n-L+1]\right]^{T}$ :

$$
\boldsymbol{x}[n]=\boldsymbol{H}[n] \boldsymbol{s}[n],
$$

where if and $M$ is the number of EMG channels, $\boldsymbol{H}$ is an $M \times N(L-1)$ mixing matrix of MUAP shapes. In practice to ensure numerical stability the extension factor $K$ is used to extend $\boldsymbol{x}[n]$ as well, effectively increasing its size to $M(K-1)$, the size of $\boldsymbol{s}[n]$ to $N(L+K-1)$, and the size of $\boldsymbol{H}[n]$ to $K M \times N(L+K-1)$. In the simplest implementation of the method the MUAP shapes are assumed to be constant, corresponding to a constant mixing matrix $\boldsymbol{H}$.

Based on the above equation we can define the activity index in the following manner: 


$$
\gamma[n]=\boldsymbol{x}^{T}[n] \boldsymbol{C}_{\boldsymbol{x} \boldsymbol{x}}{ }^{-1} \boldsymbol{x}[n]=\boldsymbol{s}^{T}[n] \boldsymbol{H}^{T}\left(\boldsymbol{H}^{T}\right)^{-1} \boldsymbol{C}_{\boldsymbol{s} \boldsymbol{s}}{ }^{-1} \boldsymbol{H}^{-1} \boldsymbol{H} \boldsymbol{s}[n]=\boldsymbol{s}^{T}[n] \boldsymbol{C}_{\boldsymbol{s s}}{ }^{-1} \boldsymbol{s}[n],
$$

where $\boldsymbol{C}_{\boldsymbol{x} \boldsymbol{x}}$ and $\boldsymbol{C}_{\boldsymbol{s} \boldsymbol{s}}$ stand for the correlation matrices of all observations of $x$ and $s$ respectively. From (A.3) it can be seen that large values of the activity index $\gamma[n]$ correspond to time instances, in which $s$ contained at least one spike. Moreover, if we replace the first term $\boldsymbol{x}^{T}[n]$ in (A.3) with $\boldsymbol{x}^{T}\left[n_{0}\right]$, using an $n_{0}$ for which $s_{j}\left[n_{0}\right]=1$, then for each time instance $n$ where only the $j$-th source is active it is true that

$$
\gamma_{n_{0}}[n]=\boldsymbol{x}^{T}\left[n_{0}\right] \boldsymbol{C}_{\boldsymbol{x} \boldsymbol{x}}{ }^{-1} \boldsymbol{x}[n]=\boldsymbol{s}^{T}\left[n_{0}\right] \boldsymbol{C}_{\boldsymbol{s s}}{ }^{-1} \boldsymbol{s}[n]=s_{j}[n]
$$

Based on (A.4) we can theoretically reconstruct each spike train by finding time instances in which only one spike train is active. The CKC method employs an optimization process in which spike trains are iteratively reconstructed and separated from superimposed MUAPs (see [19] for details).

Note that the noise is neglected in all above calculations. Adding a noise component $\boldsymbol{\omega}[n]$ to (A.2) would result in the noise covariance $\boldsymbol{C}_{\boldsymbol{\omega} \boldsymbol{\omega}}$ appearing in (A.3), reducing the accuracy in the compensation of $H$.

It is also worth noting that (A.2) is the mathematical description of the blind source separation problem, i.e. the estimation of sources $\boldsymbol{s}[n]$ from $\boldsymbol{x}[n]$ without the knowledge of $\boldsymbol{H}[n]$. Thus, general blind source separation methods, such as ICA, can also be used for EMG decomposition (Chen and Zhou 2016). In Sections 3.3 and 3.5 a novel blind source separation method specifically developed for EMG decomposition was used to acquire neural information from the surface EMG (Negro et al. 2016). 


\section{B Detailed results of Section 3.4}

This section contains the detailed results of Study 4 described in Section 3.4. The following comparisons were made using three sets of one-way ANOVAs for all subjects and conditions separately, using the following factors: feature, ramp duration and movement range.

\section{B.1 Feature}

For the largest ramp duration (the slowest speed), TD statistically significantly outperformed DSC for all but three subjects, while $\mathrm{DSC}_{\mathrm{RES}}$ and $\mathrm{DSC}_{\mathrm{MOD}}$ outperformed TD for all subjects except subject A3, where there was no statistically significant difference (Figure B.1). There was a significant difference between $\mathrm{DSC}_{\mathrm{RES}}$ and $\mathrm{DSC}_{\mathrm{MOD}}$ for subjects A1, A4 and A5.

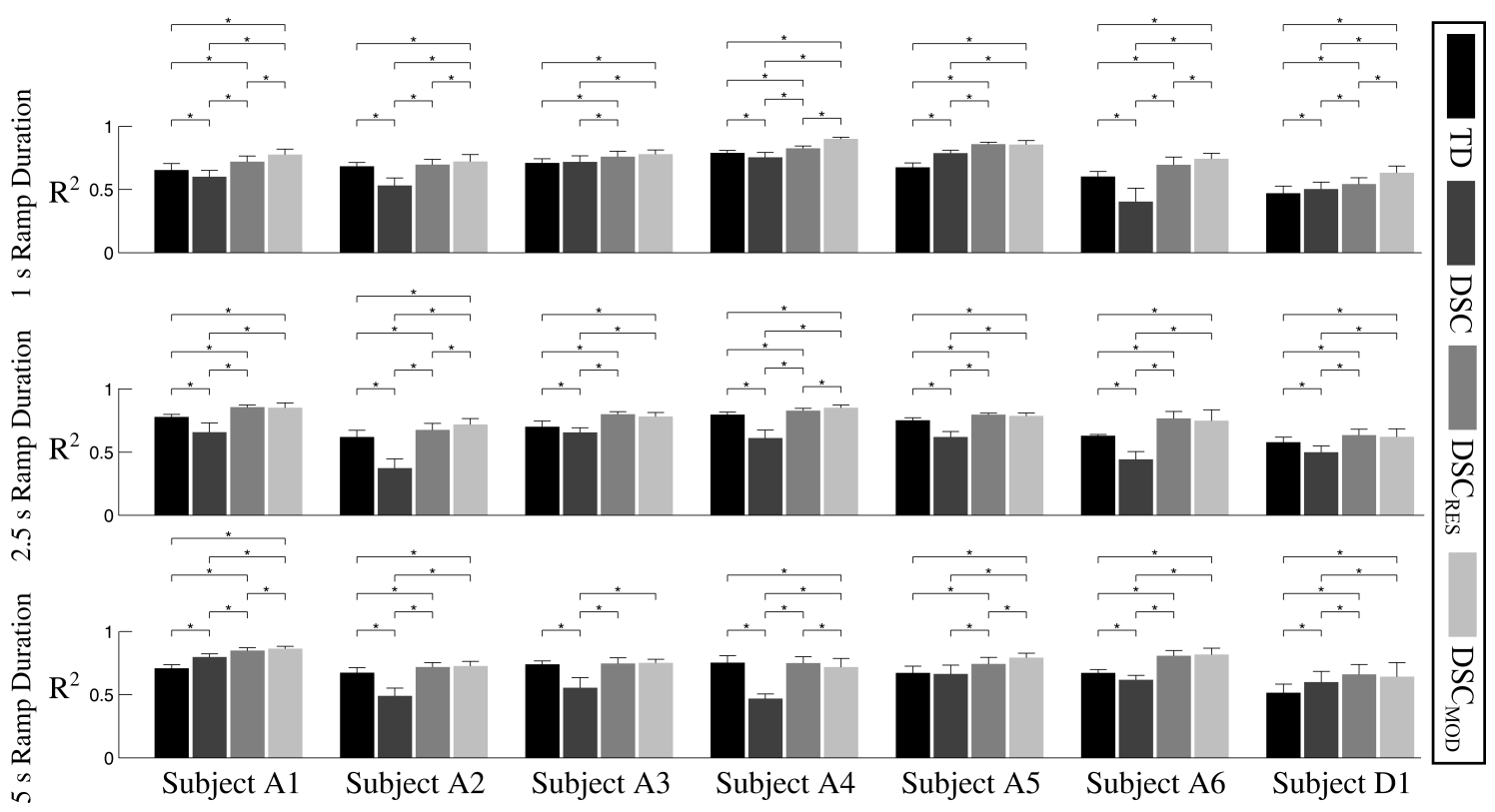

Figure B.1: Performance comparison between features at the full range of movement for different subjects and ramp durations. Bars with asterisks indicate statistically significant differences. This figure also appeared in Kapelner, Jiang, and Farina (2016).

For the 2.5-s ramp duration TD outperformed DSC, while $\mathrm{DSC}_{\mathrm{RES}}$ and $\mathrm{DSC}_{\mathrm{MOD}}$ outperformed TD. For two subjects DSC ${ }_{\text {MOD }}$ performed statistically significantly better than $\mathrm{DSC}_{\mathrm{RES}}$.

For the shortest ramp duration (the fastest speed), DS performed statistically significantly better than TD for 3 subjects including D1, and worse for 4 subjects. DSC $\mathrm{RES}_{\text {and }}$ DSC $_{\text {MOD }}$ outperformed TD in, while for 5 subjects including D1 DSC MOD $_{\text {outperformed }}$ $\mathrm{DSC}_{\mathrm{RES}}$.

In the $20 \%$ movement range, DSC set outperformed TD in all subjects except A5 and A6 at the largest ramp duration, and A2 at the shortest (Figure B.2). At the largest ramp duration $\mathrm{DSC}_{\mathrm{RES}}$ and $\mathrm{DSC}_{\mathrm{MOD}}$ outperformed TD consistently, the performance of DSC compared to the other spike based features varied across subjects. 


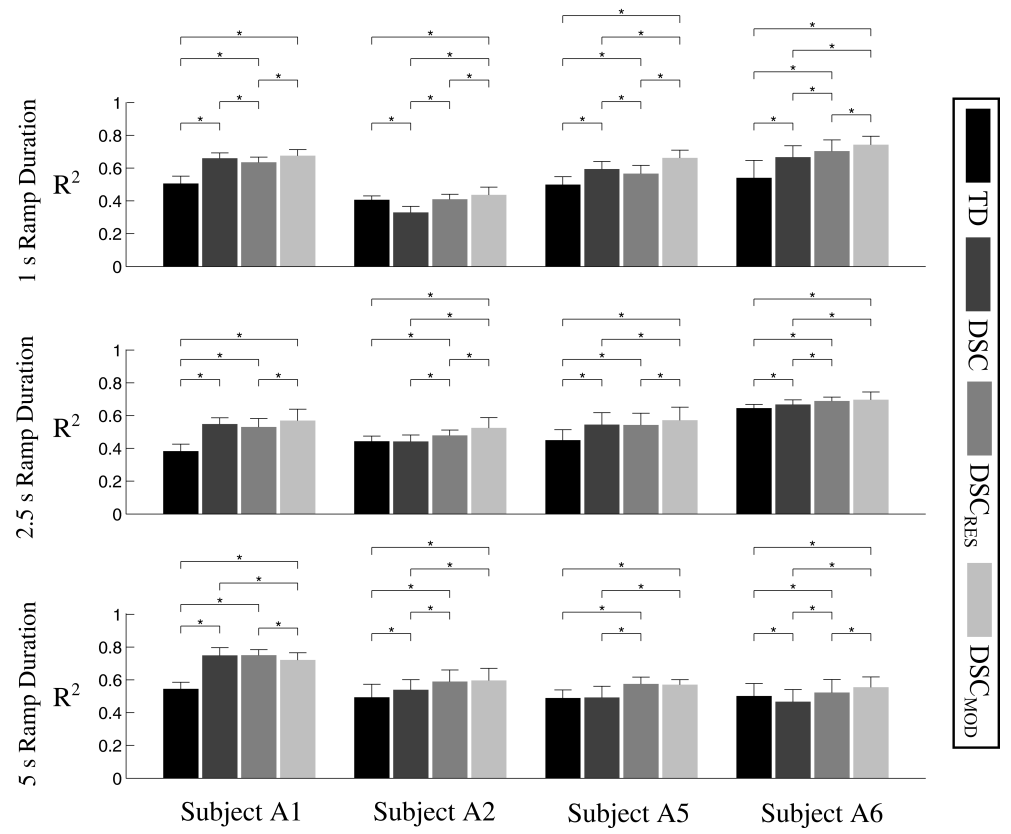

Figure B.2: Performance comparison between features at the 20\% movement range for different subjects and ramp durations. Bars with asterisks indicate statistically significant differences. This figure also appeared in Kapelner, Jiang, and Farina (2016).

For the 2.5-s ramp the order of increasing performance was TD, DSC, DSC RES and $\mathrm{DSC}_{\mathrm{MOD}}$, except for subject A1 where $\mathrm{DSC}_{\mathrm{MOD}}$ did not outperform DSC, and subject A2 where there was no statistically significant difference between $\mathrm{DSC}_{\mathrm{RES}}$ and DSC.

At the fastest speed the order of performance was the same except for subject A2 where DSC performed worse than TD, and for $\mathrm{A} 1$ and $\mathrm{A} 5$ where $\mathrm{DSC}_{\mathrm{RES}}$ performed worse than DSC.

\section{B.2 Ramp duration}

There was no consistent performance pattern with respect to ramp duration across subjects and features (Figure B.3). The speed dependence was different for each subject and feature. For the DSC feature set, subjects A1, A6 and D1 exhibited increasing performance with decreasing movement speed. The rest of the subjects showed the opposite. These patterns were mostly eliminated in the $\mathrm{DSC}_{\mathrm{RES}}$ and $\mathrm{DSC}_{\mathrm{MOD}}$ feature sets exhibiting similar differences to TD, or no significant difference for subjects A2 and D1.

Consistent speed dependence for the TD feature set for reduced movement range was also not observed (Figure B.4), similarly to the full movement range. However, the performance changed with speed more consistently across features in this movement range, especially for subject A2. 


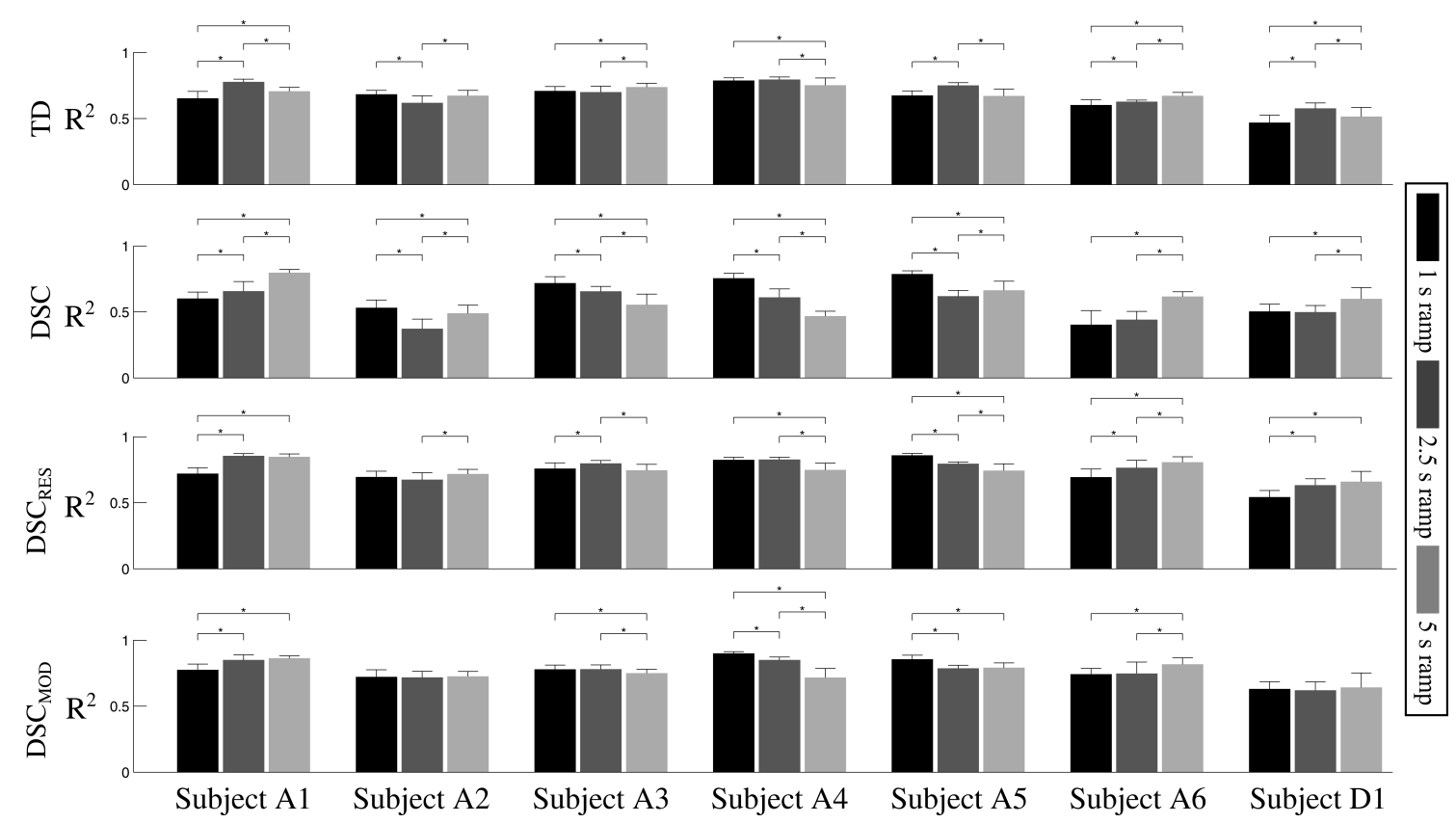

Figure B.3: Performance comparison between ramp durations at the full movement range for different subjects and features. Bars with asterisks indicate statistically significant differences. This figure also appeared in Kapelner, Jiang, and Farina (2016).

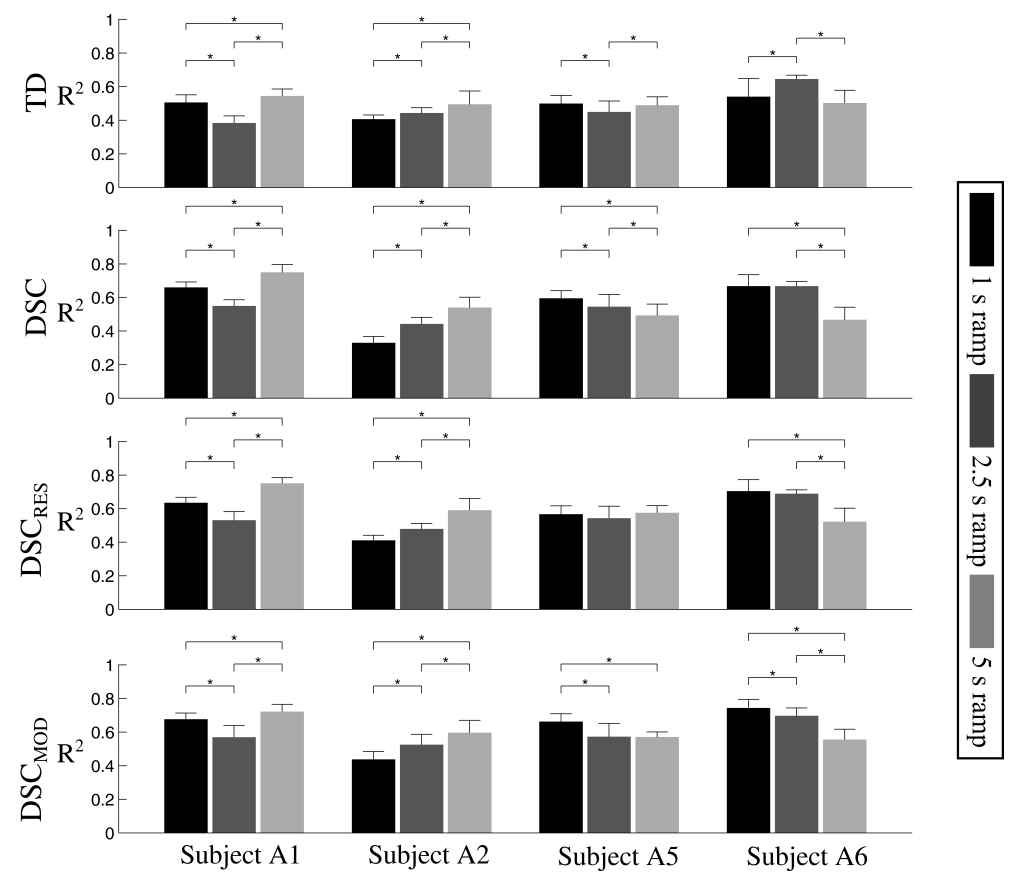

Figure B.4: Performance comparison between ramp durations at the $20 \%$ movement range for different subjects and features. Bars with asterisks indicate statistically significant differences. This figure also appeared in Kapelner, Jiang, and Farina (2016). 


\section{B.3 Movement range}

The performance of all features was statistically significantly worse for the reduced movement range, with some exceptions. TD performed better for the reduced movement range for subject A6 in the $2.5 \mathrm{~s}$ ramps. DSC showed the same results for subjects A1, A2 and A5 at different speeds inconsistently (Figure B.5). For the other two feature sets the results were similar to those of TD.
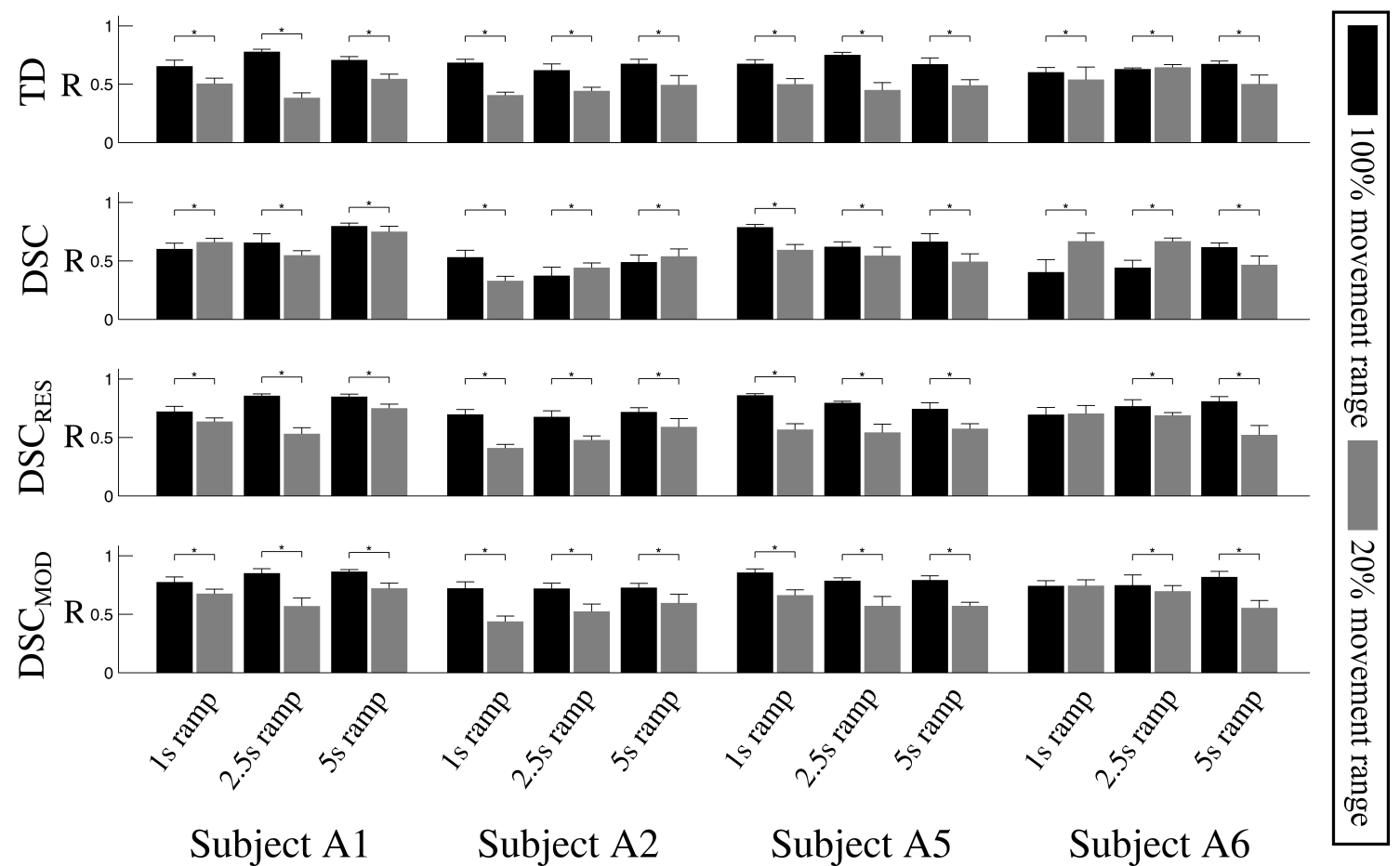

Figure B.5: Performance comparisons between the full and the reduced movement range for all subjects, ramp durations and features. Bars with asterisks indicate statistically significant differences. This figure also appeared in Kapelner, Jiang, and Farina (2016). 